University of Vermont

UVM ScholarWorks

2014

\title{
Nonlinear Ball Chain Waveguides For Acoustic Emission And Ultrasound Sensing Of Ablation
}

Stephen Herbert Pearson

University of Vermont

Follow this and additional works at: https://scholarworks.uvm.edu/graddis

Part of the Acoustics, Dynamics, and Controls Commons, and the Aerospace Engineering Commons

\section{Recommended Citation}

Pearson, Stephen Herbert, "Nonlinear Ball Chain Waveguides For Acoustic Emission And Ultrasound Sensing Of Ablation" (2014). Graduate College Dissertations and Theses. 256.

https://scholarworks.uvm.edu/graddis/256

This Thesis is brought to you for free and open access by the Dissertations and Theses at UVM ScholarWorks. It has been accepted for inclusion in Graduate College Dissertations and Theses by an authorized administrator of UVM ScholarWorks. For more information, please contact scholarworks@uvm.edu. 


\title{
NONLINEAR BALL CHAIN WAVEGUIDES FOR ACOUSTIC EMISSION AND ULTRASOUND SENSING OF ABLATION
}

\author{
A Thesis Presented \\ by \\ Stephen H. Pearson \\ to \\ The Faculty of the Graduate College \\ of \\ The University of Vermont \\ In Partial Fulfillment of the Requirements \\ for the Degree of Master of Science \\ Specializing in Mechanical Engineering
}

October, 2014 
Accepted by the Faculty of the Graduate College, The University of Vermont, in partial fulfillment of the requirements for the degree of Master of Science specializing in Mechanical Engineering.

Thesis Examination Committee:

Advisor

Dryver Huston, Ph.D.

Douglas Fletcher, Ph.D.

Chairperson

George Pinder, Ph.D.

Dean, Graduate College

Cynthia J. Forehand, Ph.D.

Date: May 23, 2014 


\begin{abstract}
Harsh environment acoustic emission and ultrasonic wave sensing applications often benefit from placing the sensor in a remote and more benign physical location by using waveguides to transmit elastic waves between the structural location under test and the transducer. Waveguides are normally designed to have high fidelity over broad frequency ranges to minimize distortion - often difficult to achieve in practice. This thesis reports on an examination of using nonlinear ball chain waveguides for the transmission of acoustic emission and ultrasonic waves for the monitoring of thermal protection systems undergoing severe heat loading, leading to ablation and similar processes. Experiments test the nonlinear propagation of solitary, harmonic and mixed harmonic elastic waves through a copper tube filled with steel and elastomer balls and various other waveguides. Triangulation of pencil lead breaks occurs on a steel plate. Data are collected concerning the usage of linear waveguides and a water-cooled linear waveguide. Data are collected from a second water-cooled waveguide monitoring Atmospheric Reentry Materials in UVM's Inductively-Coupled Plasma Torch Facility.
\end{abstract}

The motion of the particles in the dimer waveguides is linearly modeled with a three ball and spring chain model and the results are compared per particle. A theoretical nonlinear model is presented which is capable of exactly modeling the motion of the dimer chains. The shape of the waveform propagating through the dimer chain is modeled in a sonic vacuum. Mechanical pulses of varying time widths and amplitudes are launched into one end of the ball chain waveguide and observed at the other end in both time and frequency domains. Similarly, harmonic and mixed harmonic mechanical loads are applied to one end of the waveguide. Balls of different materials are analyzed and discriminated into categories. A copper tube packed with six steel particles, nine steel or marble particles and a longer copper tube packed with 17 steel particles are studied with a frequency sweep. The deformation experienced by a single steel particle in the dimer chain is approximated. Steel ball waveguides and steel rods are fitted with piezoelectric sensors to monitor the force at different points inside the waveguide during testing.

The corresponding frequency responses, including intermodulation products, are compared based on amplitude and preloads. A nonlinear mechanical model describes the motion of the dimer chains in a vacuum. Based on the results of these studies it is anticipated that a nonlinear waveguide will be designed, built, and tested as a possible replacement for the high-fidelity waveguides presently being used in an Inductively Coupled Plasma Torch facility for high heat flux thermal protection system testing. The design is intended to accentuate acoustic emission signals of interest, while suppressing other forms of elastic wave noise. 


\section{ACKNOWLEDGEMENTS}

I would like to take this time to thank my parents, grandparents, and family for all the times they have stood by me; and to thank Dr. Dryver Huston for all his help throughout my Masters Degree. This work was supported by NASA Cooperative Agreement \#NNX11AM07A. 


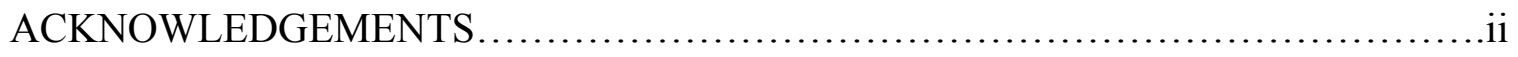

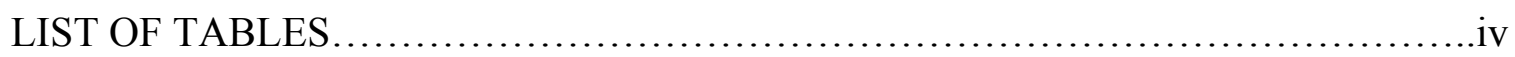

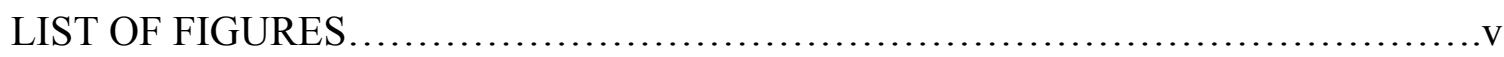

CHAPTER ONE: INTRODUCTION..........................................

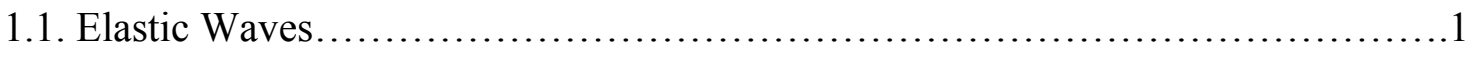

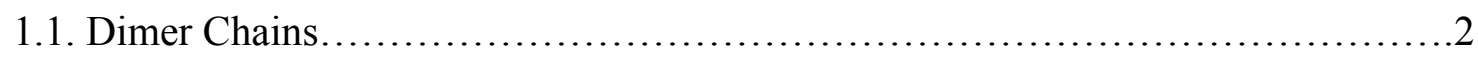

CHAPTER TWO: FLAT PLATE WAVEGUIDE TESTS $\ldots \ldots \ldots \ldots \ldots \ldots \ldots \ldots \ldots \ldots . \ldots \ldots$

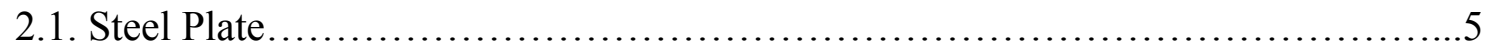

2.1.1. Acoustic Classification Features..............................................

2.1.2. Acoustic Emissions Events...............................................

2.1.3. AEWin Triangulation of Acoustic Emissions (AE) Events.............................8

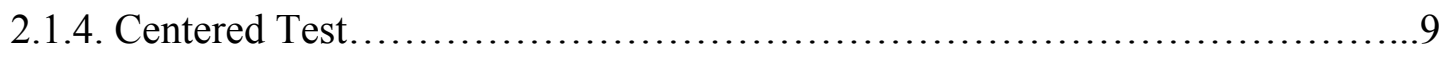

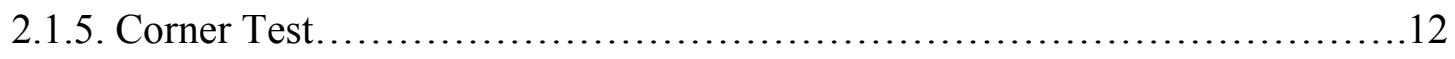

2.2. Pencil Lead Test on Water-cooled Waveguide...................................13

2.2.1. Section Results...................................................... 15

CHAPTER THREE: LINEAR WAVEGUIDE EXPERIMENTS ......................16

3.1. Water-cooled Waveguide Experiment......................................16

3.1.1. Section Results.................................................... 18

3.1.2. Linear Waveguide Testing Conclusion .................................22

3.2. Uncooled Waveguide Experiment...........................................23

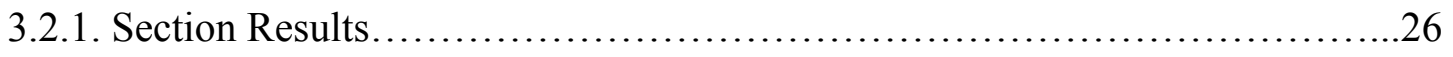


3.3. Butane Torch Excitation Experiment - Uncooled Waveguide...................30

3.3.1. Section Results.................................................. 31

3.3.2. Conclusion of the Butane Torch Excitation Experiment with Uncooled

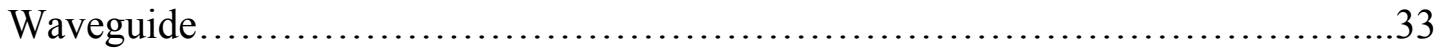

CHAPTER FOUR: INDUCTIVELY-COUPLED PLASMA TORCH LAB DATA

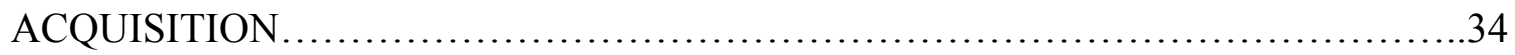

4.1. Test Setup and Procedure............................................. 34

4.2. Section Results................................................... 38

4.2.1. Data Analysis...................................................... 41

CHAPTER FIVE: WAVEGUIDE THEORY OF OPERATION $\ldots \ldots \ldots \ldots \ldots \ldots \ldots \ldots \ldots . . . . . . . .45$

5.1. Linear Elastic Ball Model..............................................45

5.2. Hertz Law for Nonlinear Interaction....................................47

5.2.1. Modified Model for Hertzian Interaction.............................48

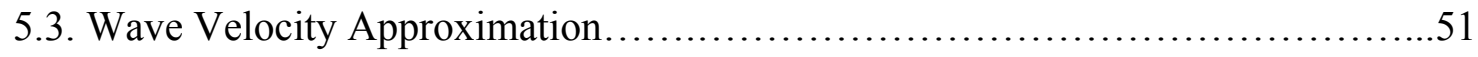

5.4. Reciprocal Approximation...............................................53

CHAPTER SIX: NONLINEAR WAVEGUIDE DISCRIMINATION.................54

6.1. Test Setup and Procedure.............................................. 54

6.2. Chapter Results....................................................57

6.3. Trial Discrimination and Analysis.....................................62

6.4. Conclusion of the Nonlinear Waveguide Discrimination......................64

6.5. Waveguide Comparison...............................................65

CHAPTER SEVEN: FREQUNECY SWEEPS AND PULSES $\ldots \ldots \ldots \ldots \ldots \ldots \ldots \ldots \ldots . \ldots 1$ 
7.1. Function Generator Sweep................................................ 71

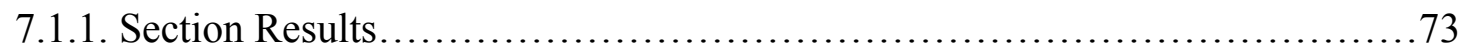

7.2. Frequency Sweeps With Precompression......................................74

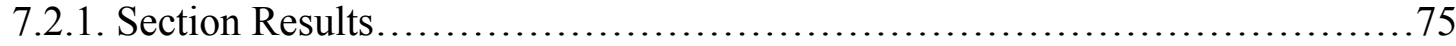

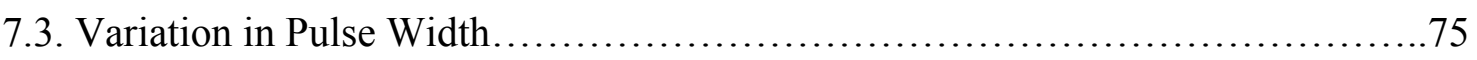

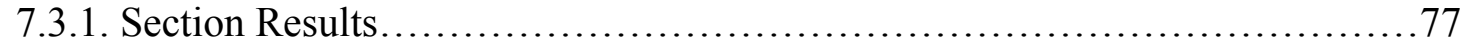

CHAPTER EIGHT: DEFORMATION_............................................78

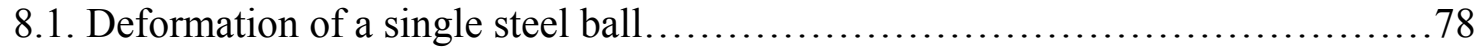

CHAPTER NINE: FORCE SENSOR EXPERIMENTS ..............................80

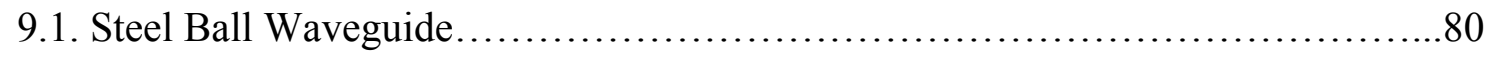

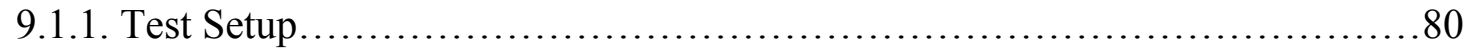

9.1.2. Section Results................................................... 82

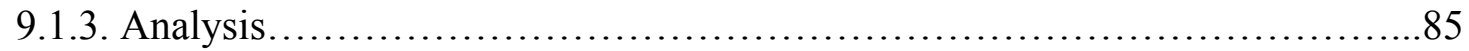

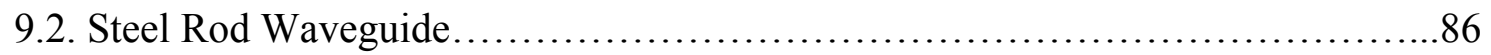

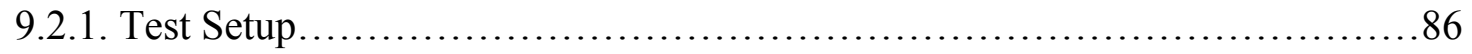

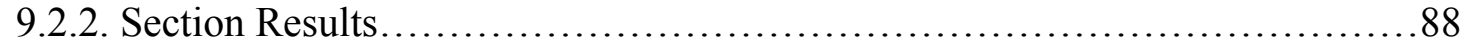

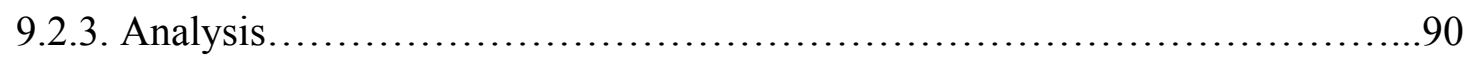

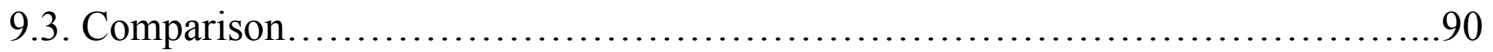

CHPATER TEN: WAVEGUIDE ACTUATION......................................91

10.1. Steel Rod................................................................ 91

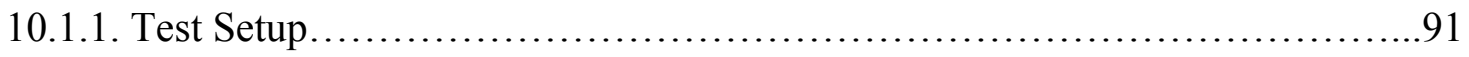

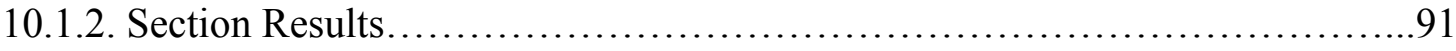


CHAPTER TEN: CONCLUSION.

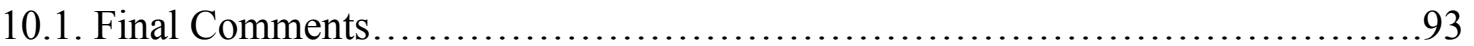

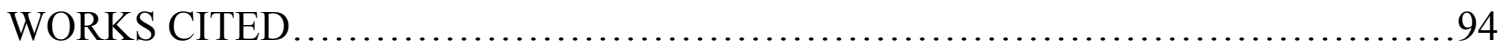

APPENDIX A: CALCULATIONS...................................................... 95 


\section{LIST OF TABLES}

Table

Page

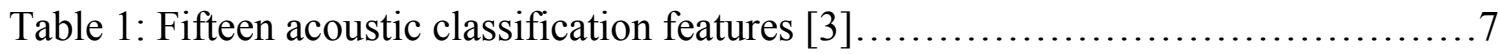

Table 2: Trial numbers, corresponding flow rates, and color labels per flow

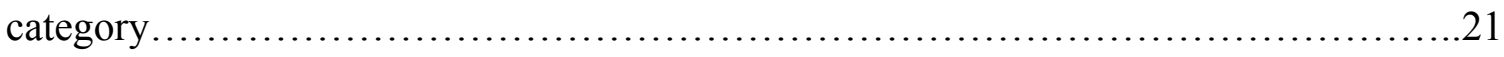

Table 3: Four discriminated groups of trials with the trials most frequent flow rate.....22

Table 4: Graphite sample test data, plasma torch................................ 36

Table 5: Young's modulus and damping ratio for the five materials [7] [8] ............55 


\section{LIST OF FIGURES}

Figure

Page

Figure 1: Test samples for monitoring material performance in a high temperature plasma stream 4

Figure 2: Four R15 $\alpha$ sensors coupled to an aluminum plate in rectangular formation......5

Figure 3: Demonstration of a pencil lead test; note, hand is elevated from plate............8

Figure 4: Three lead breaks (red) centered on the plate (white) with four sensors (green) and one outlier...............................................................

Figure 5: Amplitude vs. time data for the three triangulated events in figure $4 \ldots \ldots \ldots . .11$

Figure 6: Expanded amplitude peak, second event from figure $5 \ldots \ldots \ldots \ldots \ldots \ldots \ldots \ldots \ldots \ldots \ldots$

Figure 7: Four lead breaks in rectangular formation near the sensors...................13

Figure 8: The water-cooled waveguide firmly clamped to a granite counter with its water intake connected to a faucet and an "exhaust" tube down the drain.....................14

Figure 9: Two-sensor amplitude vs. time data, lead test on the water-cooled waveguide with running water........................................................ 15

Figure 10: Four sensors spaced evenly along the copper pipe of water-cooled

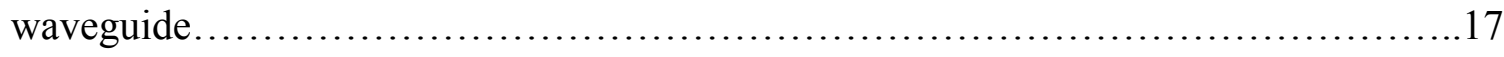

Figure 11: Two-sensor adjacent T-connection configuration, similar to the configuration that will be monitoring the waveguide in the plasma facility $\ldots \ldots \ldots \ldots \ldots \ldots \ldots \ldots \ldots . \ldots \ldots$

Figure 12: Glyph plots of the median data from the trials.........................19 
Figure 13: Grouped glyph plots, clustered by the color representing the most prevalent flow rate of the group, numerical and categorical labels carried from previous figure....20

Figure 14: Glyph plot variable key........................................21

Figure 15: Waveguide 2 with a graphite sample in the holder, right..................23

Figure 16: The graphite sample from figure 15 out of the holder......................24

Figure 17: Two function generators on a housing with four linear amplifiers; top left is the chirp waveform generator; top right is the TTL trigger; bottom outside modules in the housing are the units used for the linear scaling of the waveform $\ldots \ldots \ldots \ldots \ldots \ldots \ldots \ldots . \ldots \ldots$

Figure 18: Actuated sensors placed firmly against the graphite sample................25

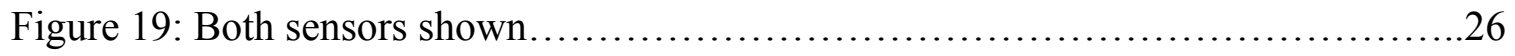

Figure 20: Left, the scaled chirp-waveform cycling twice in one second; right, the resulting AE signal from the second sensor.....................................26

Figure 21: Zoomed in versions of the waveforms, in the excited area directed by the

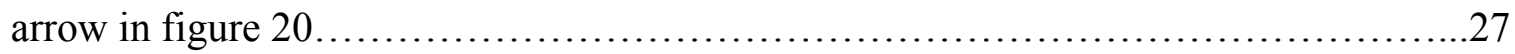

Figure 22: View down the uncooled waveguide, butane test........................29

Figure 23: The butane torch is applied to cork sample while AEWin is collecting data...30 Figure 24: Close up of the burning cork sample................................ 30

Figure 25: Charred cork.................................................. 31

Figure 26: AE data from two ignitions of the butane torch, held directly overhead of the

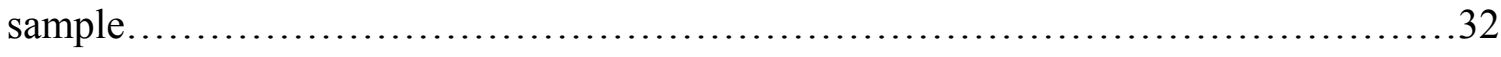

Figure 27: AE data from a trial where the torch is ignited and then moved over the head of the sample. .33 


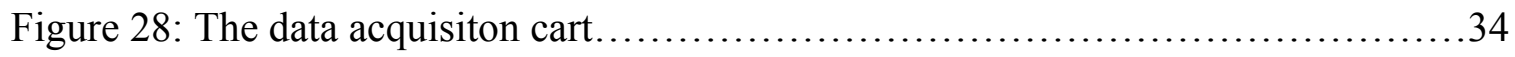

Figure 29: UVM's Inductively Coupled Plasma ICP Torch.........................35

Figure 30: Close up of sensor Placement, brass water-cooled waveguide in the ICP torch

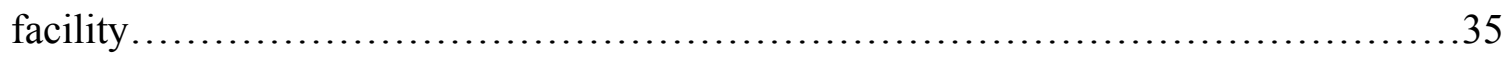

Figure 31: Absolute energy (aj) vs time (s)..................................... 37

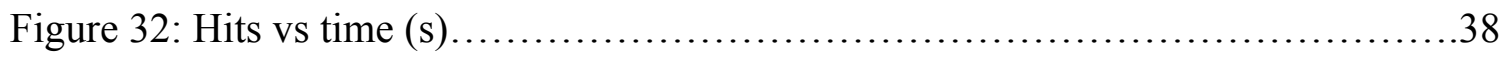

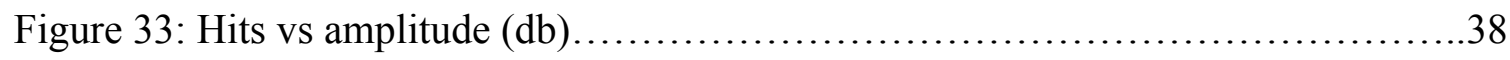

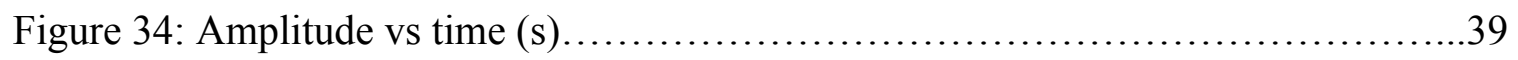

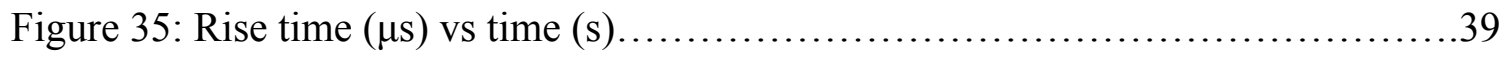

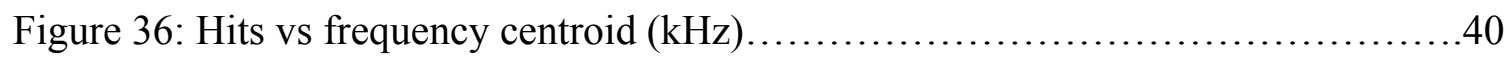

Figure 37: Labeled glyph plots of the seven trials in the plasma facility; yellow, 10 SLMP Ar / 30 SLMP N; orange, 40 SLMP N; green, 30 SLMP Ar / 10 SLMP N; cyan, 40 SLMP Ar............................................................ 41

Figure 38: Classified trials with group labels ; orange, $\mathrm{N}$ based; blue, Ar based..........41

Figure 39: Graphite sample.............................................42

Figure 40: Inside the plasma torch, excited graphite sample........................43

Figure 41: A one-dimensional three ball and spring linear system..................45

Figure 42: The positions of seven balls in an excited linear spring-ball chain vs. time...46 Figure 43: The positions of balls one (blue), two (red), and three (green) in the nonlinear model vs. time........................................................ 50

Figure 44: Shape of the wave inside a chain vs. axis of wave propagation..............52 
Figure 45: The experimental setup with actuating transducer, left; and sensing transducer, right .56

Figure 46: Voltage vs. time graphs for a portion of the chirp waveform $<1>$ and the sensing transducer $<2>$ with corresponding power vs. frequency graphs for the all steel ball trial.

Figure 47: Resulting output voltage of the receiving transducer across a one second period for the all steel ball trial. .58

Figure 48: Resulting output voltage across a one second period for the rubber ball trial. .59

Figure 49: Resulting output voltage across a one second period for the cork ball trial....59 Figure 50: Resulting output voltage across a one second period for the neoprene bal9 trial.

Figure 51: Resulting output voltage across a one second period for the marble ball trial. .60

Figure 52: Resulting output voltage across a one second period for an L-shaped waveguide with a graphite sample head and no particle chain 61

Figure 53: Glyph plots of the 15 trials for discrimination with their actual trial type......62 Figure 54: The 15 trials discriminated into groups based upon five acoustic characteristics acquired by the sensing transducer with grouping key....

Figure 55: Glyph plots of the training matrix from which 5 characteristics are used for discrimination with grouping key .64

Figure 56: Steel Ball Waveguide Experiment .66 
Figure 57: Fourier transform of the excitation waveform for the striker.

Figure 58: Fourier transform of the response from the steel ball waveguide. 67

Figure 59: Fourier transform of the response of the steel rod waveguide...............68

Figure 60: Fourier transform of the response of the steel ball waveguide with sample...69

Figure 61: $1 \mu \mathrm{Hz}$ to $500 \mathrm{kHz}$ Sweep for the nine steel ball waveguide................71

Figure 62: $1 \mu \mathrm{Hz}$ to $500 \mathrm{kHz}$ Sweep for the 17 steel ball waveguide...................72

Figure 63: $1 \mu \mathrm{Hz}$ to $500 \mathrm{kHz}$ Sweep for the nine marble ball waveguide...............72

Figure 64: $1 \mu \mathrm{Hz}$ to $500 \mathrm{kHz}$ Sweep for the six steel ball waveguide with $1.28 \mathrm{~kg}$ precompression......................................................... 74

Figure 65: $1 \mu \mathrm{Hz}$ to $500 \mathrm{kHz}$ Sweep for the six steel ball waveguide with $3.28 \mathrm{~kg}$

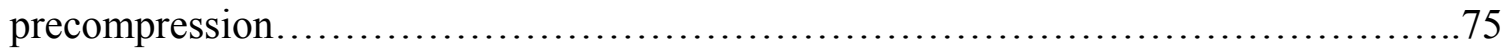

Figure 66: pulse with four-microsecond width............................... 76

Figure 67: pulse with three-microsecond width............................... 76

Figure 68: pulse with two-microsecond width................................ 77

Figure 69: pulse with one-microsecond width................................ 77

Figure 70: Peak deformation gradient of a steel ball during sinusoidal excitation........79

Figure 71: Close up of a piezoelectric sensor fixed within a steel ball ................81

Figure 72 : Close up of the testing rig with sensor labels.......................... 82

Figure 73: Results of the low drop test from piezoelectric sensor $1 \ldots \ldots \ldots \ldots \ldots \ldots \ldots . \ldots 2$

Figure 74: Results of the low drop test from piezoelectric sensor $2 \ldots \ldots \ldots \ldots \ldots \ldots \ldots . \ldots 3$

Figure 75 : Results of the high drop test from piezoelectric sensor $1 \ldots \ldots \ldots \ldots \ldots \ldots \ldots . \ldots 3$

Figure 76: Results of the high drop test from piezoelectric sensor $2 \ldots \ldots \ldots \ldots \ldots \ldots . . . .44$ 
Figure 77: Side by side comparison of the trigger time of sensor 1 and sensor 2 from the low drop test........................................................... 84

Figure 78: Results of the pencil lead test with one central sensor....................86

Figure 79: Close up of the steel rod in the testing rig ........................... 87

Figure 80: Results of the low drop test from piezoelectric sensor $1 \ldots \ldots \ldots \ldots \ldots \ldots \ldots . \ldots 8$

Figure 81: Results of the low drop test from piezoelectric sensor $2 \ldots \ldots \ldots \ldots \ldots \ldots \ldots . \ldots 8$

Figure 82: Results of the high drop test from piezoelectric sensor $1 \ldots \ldots \ldots \ldots \ldots \ldots \ldots . \ldots 9$

Figure 83: Results of the high drop test from piezoelectric sensor $2 \ldots \ldots \ldots \ldots \ldots \ldots \ldots . \ldots . \ldots . \ldots$

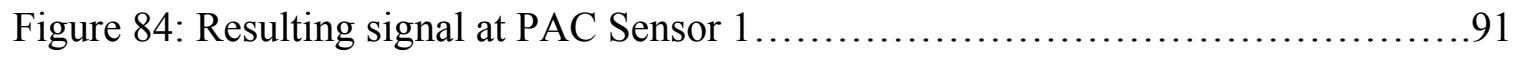

Figure 85: Resulting Fourier Transform of the signal in figure $84 \ldots \ldots \ldots \ldots \ldots \ldots \ldots . \ldots 1$

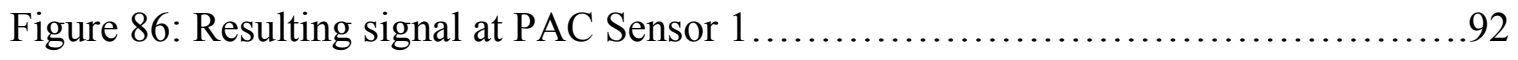

Figure 87: Resulting Fourier Transform of the signal in figure $86 \ldots \ldots \ldots \ldots \ldots \ldots \ldots . \ldots 2$ 


\section{CHAPTER ONE: INTRODUCTION}

\subsection{Elastic Waves}

The laws concerning strength of materials govern the mechanics of elastic waves in a steel rod. The wave equations that govern the motion of a vibrating steel rod are interchangeable with the equations that govern the vibration of a long string, such as the D’Alembert solution [1]. The governing equation for a straight, long prismatic rod is shown below as equation 1.

$$
-\sigma A+\left(\sigma+\frac{\partial \sigma}{\partial x} d x\right) A+q A d x=\rho A d x \frac{\partial^{2} u}{\partial t^{2}}
$$

\section{Equation 1: Governing wave equation for a long, straight prismatic rod}

The coordinate $x$ refers to a cross-section of the rod; the corresponding displacement is given by $u(x, t)$; the rod experiences a dynamic stress field of $\sigma(\mathrm{x}, \mathrm{t}) ; q(x, t)$ is a body force per unit of volume; $A$ is the cross section of the rod [1]. Assuming the tensile stress and positive, the material is elastic, the rod is homogeneous, and that there are no body forces, equation one can be reduced to equation 2 .

$$
E \frac{\partial^{2} u}{\partial x^{2}}=\rho \frac{\partial^{2} u}{\partial t^{2}}
$$

Equation 2: The wave equation 
The solution to this equation is the D'Alembert solution found as equation 3 on the following page.

$$
u(x, t)=f(x-t \sqrt{E / \rho})+g(x+t \sqrt{E / \rho})
$$

Equation 3: The D'Alembert solution

Elastic waves are deformational excitations that travel through media at some wave speed. The $P$ wave propagates through a continuum as a series of compressions with a wave speed of $[K / \rho]^{1 / 2}$, where $K$ is the bulk modulus and $\rho$ is the density of the material. The $S$ wave is a secondary wave found in excited media due to shear with isotropic medium wave speed of $[G / \rho]^{1 / 2}$. For many materials the $S$ wave speed is approximately one half of the $P$ wave speed. The Lamb waves are waves found in flat plates where the motion of the wave occurs within the plane defined by the direction of the wave and a perpendicular to the plate [1].

\subsection{Dimer Chains}

Acoustic emission (AE) and ultrasonic wave sensing are based on elastic wave vibration transmission to monitor structural conditions. One application with largely linear elastodynamic properties arises in testing the condition of structures operating in harsh environments, such as the evaluation of thermal protection systems (test sample material in figure 1, page 4) used in hypersonic spacecraft and aircraft [2]. Effective sensing often requires using waveguides to transmit the vibrations to a remote transducer. 
Most waveguides operate in a linear manner to transmit the waves with high fidelity over a broad frequency range. An alternative to the linear waveguide is the nonlinear ball chain waveguide [2]. These waveguides transmit elastic waves with shapes, speeds and wavelengths that largely depend on the size and elastic properties of the balls. It is possible that these nonlinear waveguides can be used to monitor thermal protection systems experiencing severe heat loading and ablation.

In this thesis, a series of experiments and analyses of acoustic emissions and other elastic wave propagations through linear and nonlinear ball-chain waveguides is presented. Flat plate waveguide test and copper tube waveguide tests examine behavior in largely linear contexts. Experiments are used to investigate the nonlinear propagation of harmonic and mixed harmonic elastic waves through a stack of steel balls loosely held in place by a copper tube. Wave propagation through stacks of balls of other materials is also investigated. Mechanical pulses or strikers mechanically excite the waveguides in forms that can be analyzed in both time and frequency domains. Frequency domain responses can be compared with respect to striker amplitudes and preloads.

A nonlinear mechanical model describes the motion of the dimer chains while within a vacuum. The ultimate intention is to design waveguides to accentuate acoustic emission signals, while suppressing other elastic wave noise. 


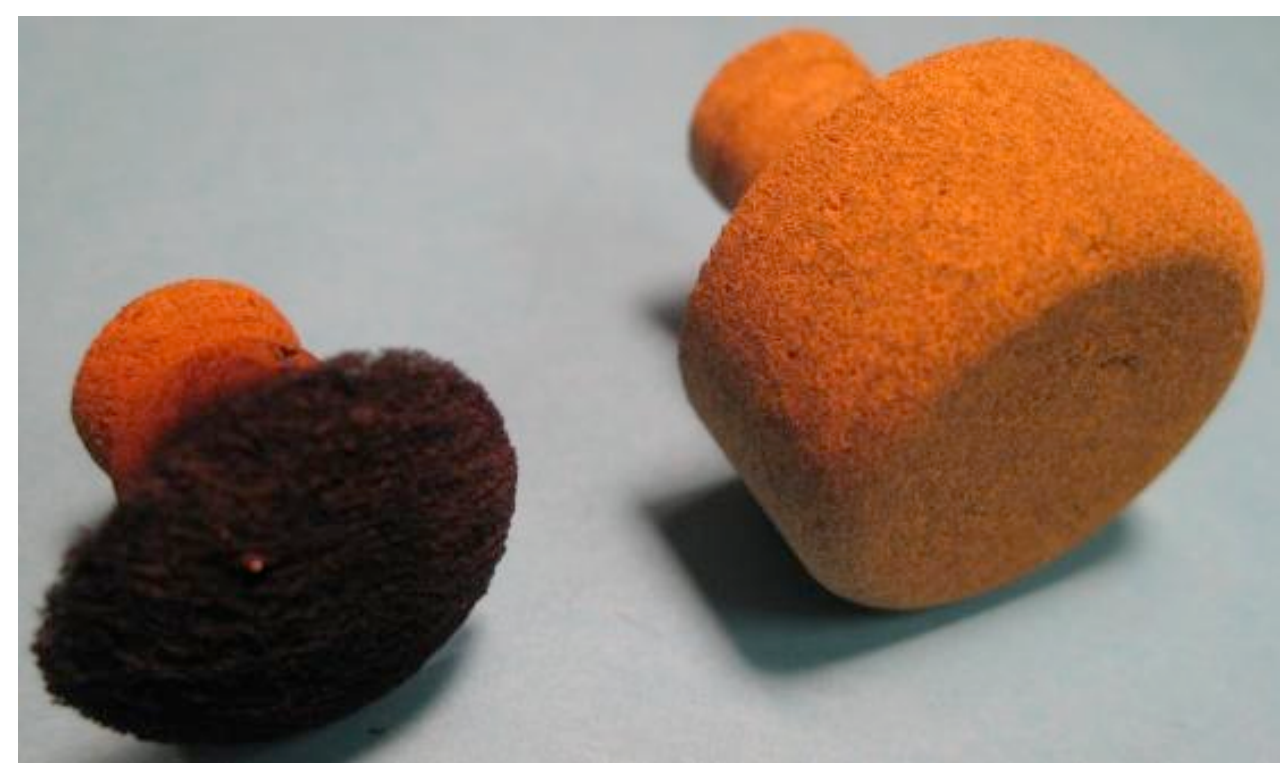

Figure 1: Test samples for monitoring material performance in a high temperature plasma stream [2] 


\section{CHAPTER TWO: FLAT PLATE WAVEGUIDE TESTS}

\subsection{Steel Plate}

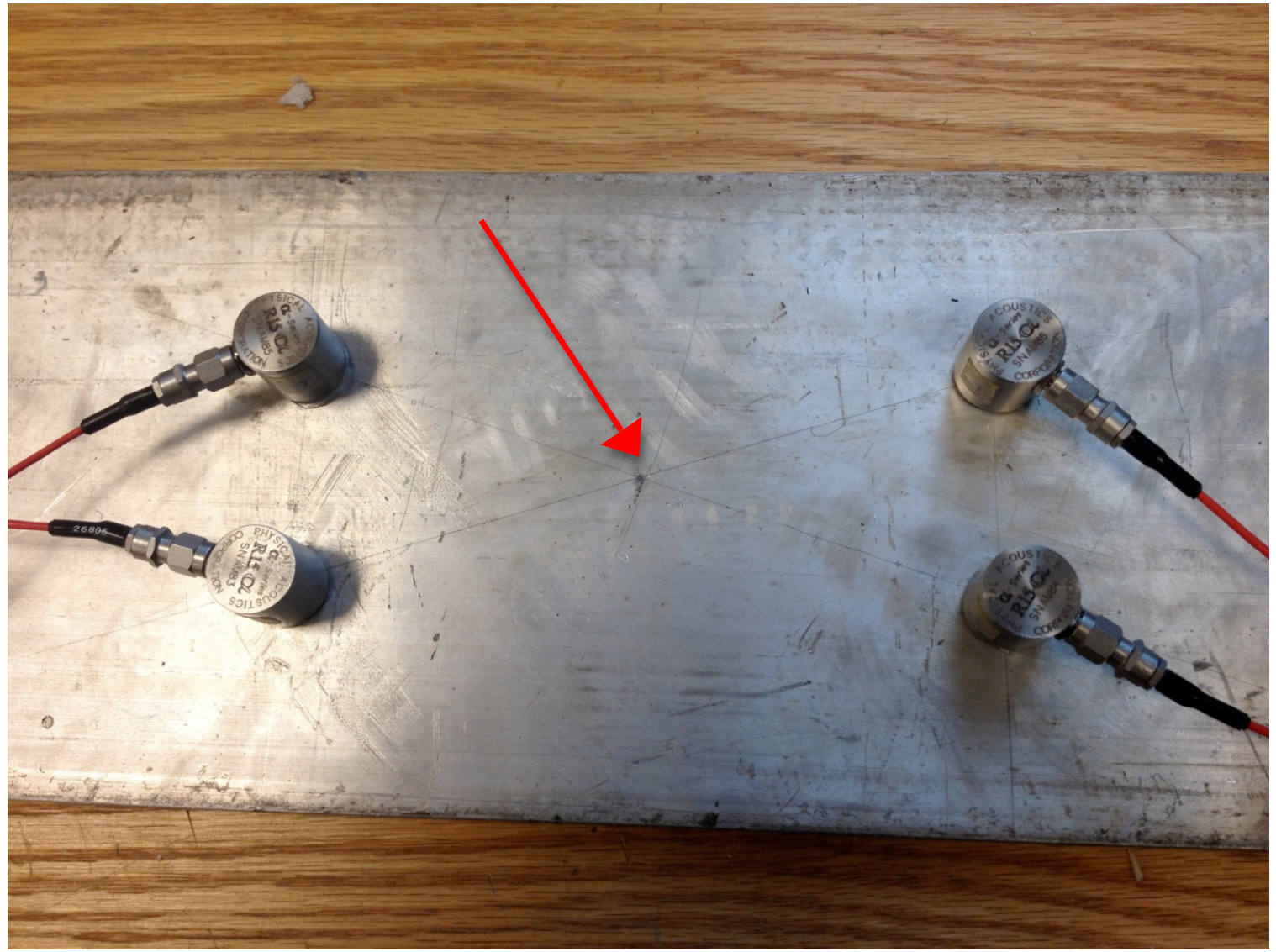

Figure 2: Four R15a sensors coupled to an aluminum plate in rectangular formation

Pencil lead break testing is a standard method of elastic waves in solids that are similar to those produced by acoustic emissions events. In a series of initial tests, pencil lead break tests were performed on a flat aluminum plate that acted as an elastic waveguide. The purpose of the lead test is to become familiar with the PAC data acquisition unit, and to obtain baseline acoustical emissions data related to the breaking lead on a metal surface. 
Four Physical Acoustic's Corporation (PAC) R15a sensors are coupled to an aluminum plate (6" x 18") with a silicone compound in the above formation. The sensors are connected to a PAC PCI-2 board through a set of four PAC 20/40/60 preamplifiers (each set $40 \mathrm{~dB}$ ); PAC AEWin software is prepared for an aluminum plate triangulation. Lead (actually graphite in a cylinder with $0.5 \mathrm{~mm}$ nominal diameter) from a mechanical pencil is broken in the center of the plate (marked by the arrow in figure 2). The breaks register as events in the software; the events are comprised of collected sets of "hits". A hit registers when the preamp output surpasses a predetermined threshold in the AEWin software [3]. 


\subsubsection{Acoustic Classification Features}

Each hit can be classified by a set of 15 quantities defined in the software seen in table 1 .

\begin{tabular}{|l|l|}
\hline Rise time & The interval from the initial threshold of signal to peak amplitude \\
\hline Count & The number of times the signal crosses the threshold during the hit \\
\hline Energy & The magnitude of energy of the entire hit \\
\hline Duration & The time between the first and last threshold crossing of the hit \\
\hline Amplitude & The peak voltage amplitude associated with the hit \\
\hline Average frequency & The mean frequency of the hit \\
\hline RMS & The root mean square value of the voltage signal across its duration \\
\hline Average signal level & A logarithmic representation of the RMS value \\
\hline Counts-to-peak & The counts before a maximum amplitude is reached during a hit \\
\hline Reverberation frequency & The most persistent frequency across a hit \\
\hline Initiation frequency & The initial frequency of a hit \\
\hline Signal strength & An integral across the duration of the rectified signal \\
\hline Absolute energy & Related to an integral of the voltage squared \\
\hline Frequency centroid & The "weighted center" of a hit's frequency spectrum \\
\hline Peak frequency & The greatest frequency \\
\hline
\end{tabular}

Table 1: Fifteen acoustic classification features [3]

\subsubsection{Acoustic Emissions Events}

The measured energy quantities depend on the associated gain of the preamplifiers and are comparable to one another when the gains are the same. Events are a series of hits all related to the same incident. A single lead test trial should ideally 
register one event in the system with the possibility of multiple hits due to internal reflections [3].

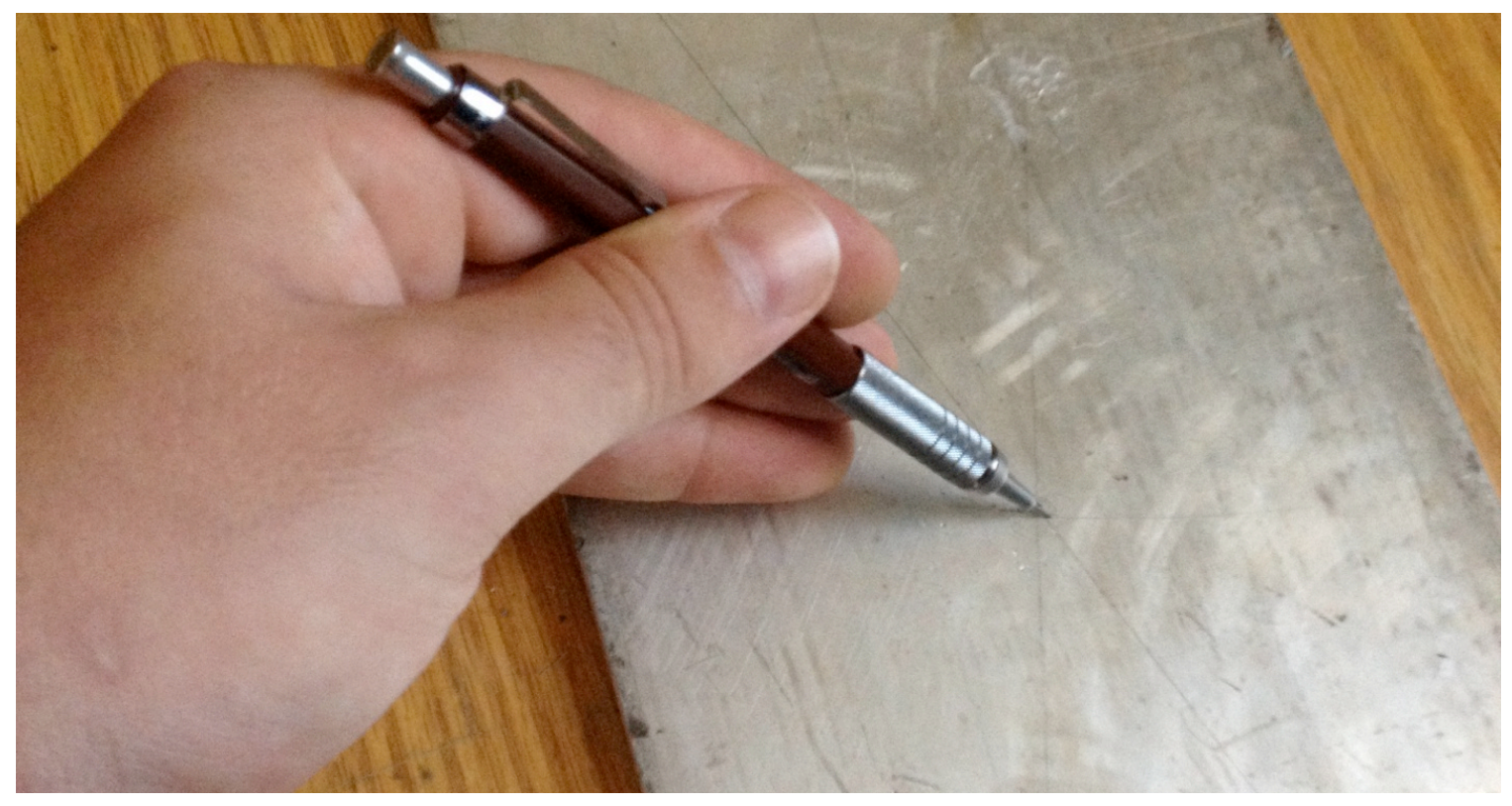

Figure 3: Demonstration of a pencil lead test; note, hand is elevated from plate

\subsubsection{AEWin Triangulation of Acoustic Emissions (AE) Events}

The AEWin software is capable of triangulating events based on the characteristics of hits with predefined sensor locations, and plate characteristics. Events are registered and displayed on a $2 \mathrm{D}$ scatter plot in respect to the locations of the four sensors and the dimensions of the plate. Figure 3 displays the triangulation results of three lead breaks intentionally placed in the center of the plate. 


\subsubsection{Centered Test}

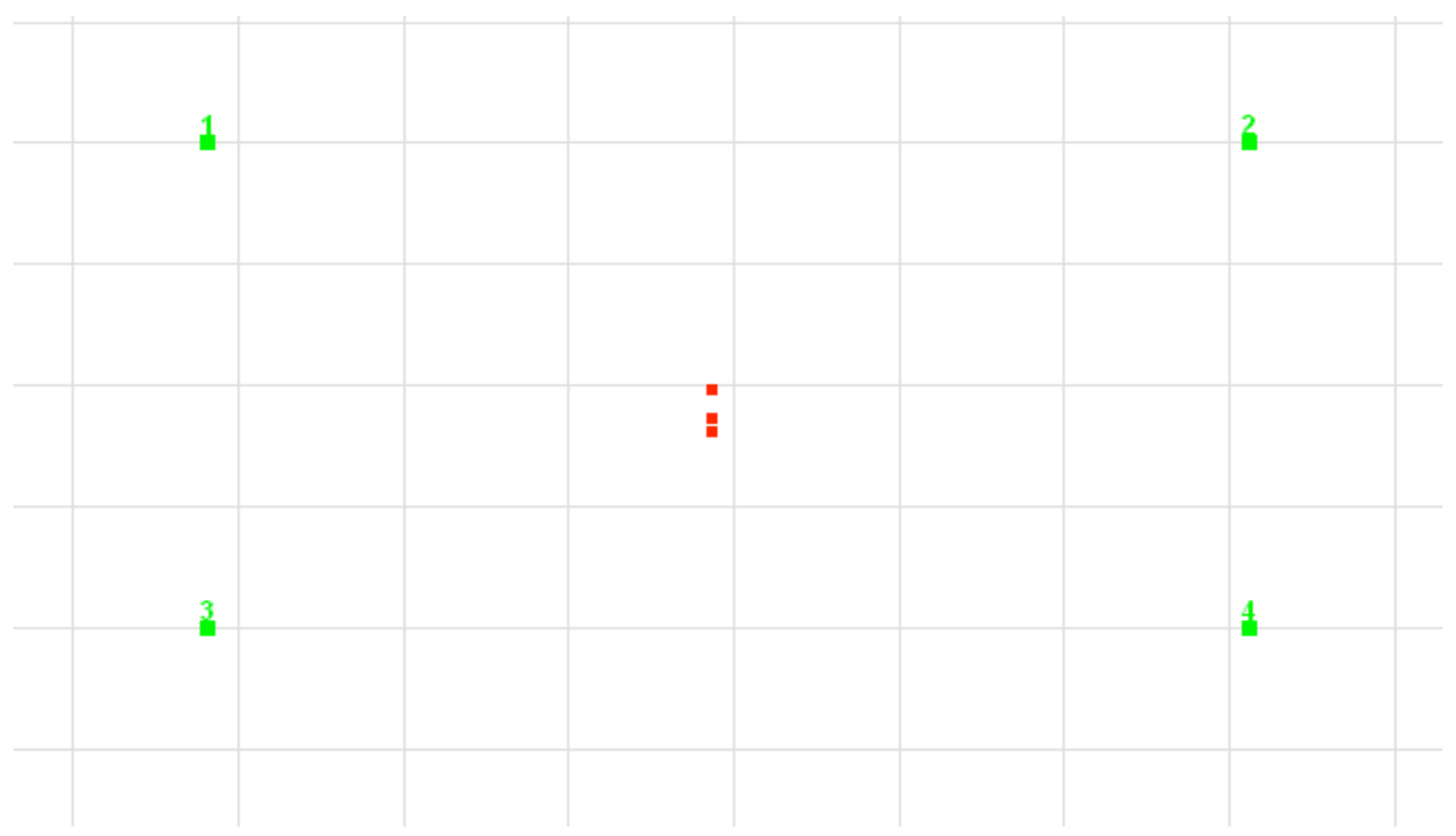

Figure 4: Three lead breaks (red) centered on the plate (white) with four sensors (green) and one outlier

The measurement of the center of the plate (estimated by connecting opposite corners) can be considered accurate to within about $+/-1.02 \mathrm{~mm}$ from its actual center along its width and $+/-3.05 \mathrm{~mm}$ from its actual center along its length with a 95\% confidence (Appendix A1). The accuracy of lead break with respect to the actual center can be considered the previous error, in addition to a $1.59 \mathrm{~mm}$ error in both directions (this additional error can be seen in the figure 3 from the last page: the tip of the mechanical pencil is slightly right of the " $\mathrm{X}$ " marking the supposed center); thus, the accuracy of the placement of a lead break can be considered to be within about $+/-2.54$ $\mathrm{mm}$ from the actual center of the plate along its width and $+/-4.57 \mathrm{~mm}$ from its actual 
center along its length with a $95 \%$ confidence. The accuracy of the placement of the sensors with respect to the actual center of the plate can be considered the error from Appendix A1 with an additional $3.18 \mathrm{~mm}$ error in both directions. Thus, the sensors are likely placed within a range defined as $+/-4.32 \mathrm{~mm}$ along the plate's width and $+/-6.35$ $\mathrm{mm}$ along its length from the calibrated placements in the software with a $95 \%$ confidence. Both the error related to the location of the lead breaks and the error related to the placement of the sensors can help to account for the events triangulation slightly left of center in figure 4 (measured center left of actual center, lead breaks left of center, sensors positions skewed left). 
Amplitude (dB)

100

75

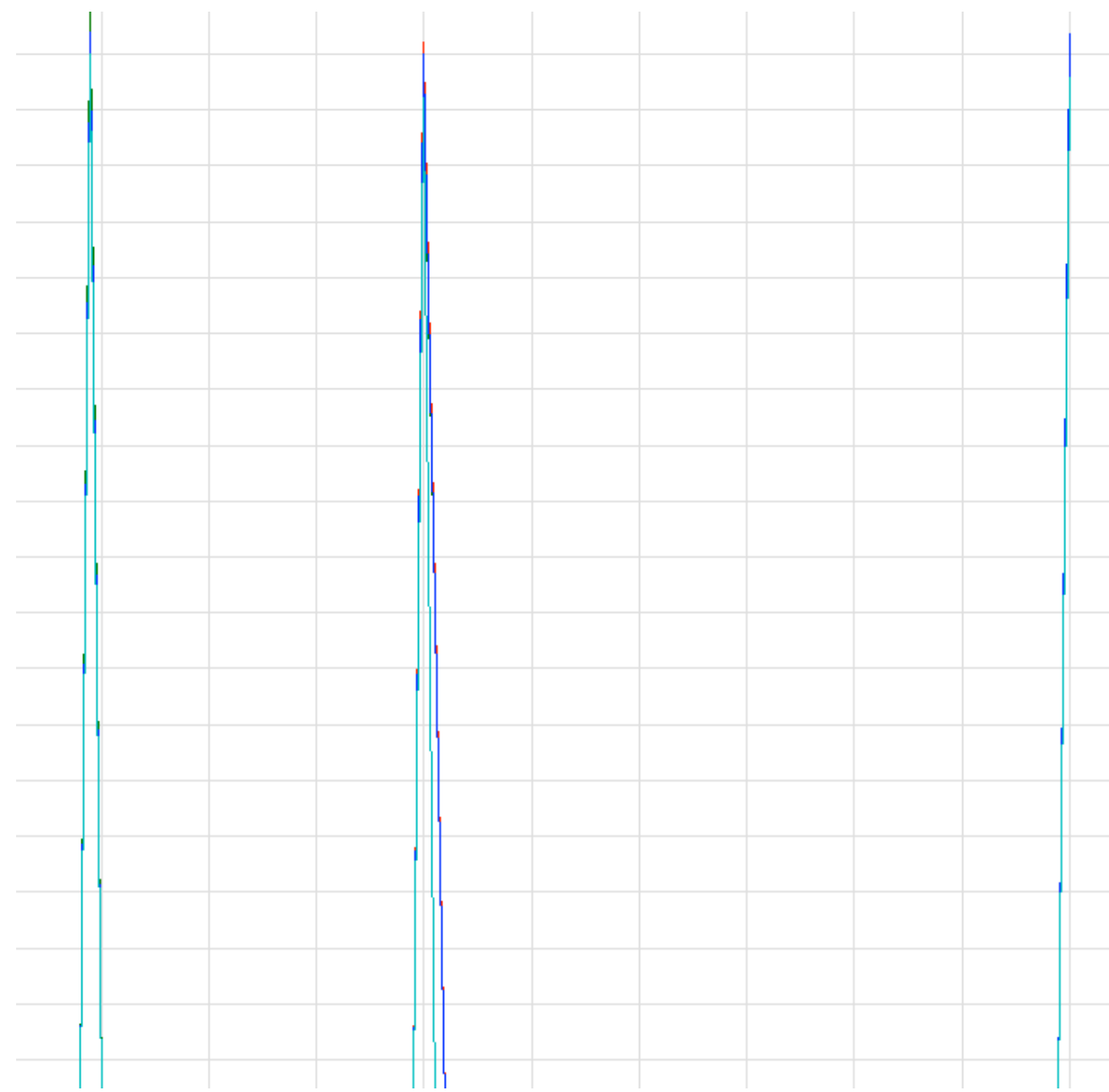

0

Time (s)

Figure 5: Amplitude vs. time data for the three triangulated events in figure 4

Figure 5 represents the voltage amplitude of each of the four sensors against time, revealing three clear events. The middle peak in the above figure is expanded bottomright; this event is the centermost event of the three events triangulated in figure 4 . The amplitude data are different for each of the sensors, directly related to the triangulation. 
Amplitude data from sensors one and three are greater than that of sensors two and four; hence, the event is triangulated left of center (sensors one and three are the leftmost sensors if figure 4). The center peak is zoomed in upon in figure 6.

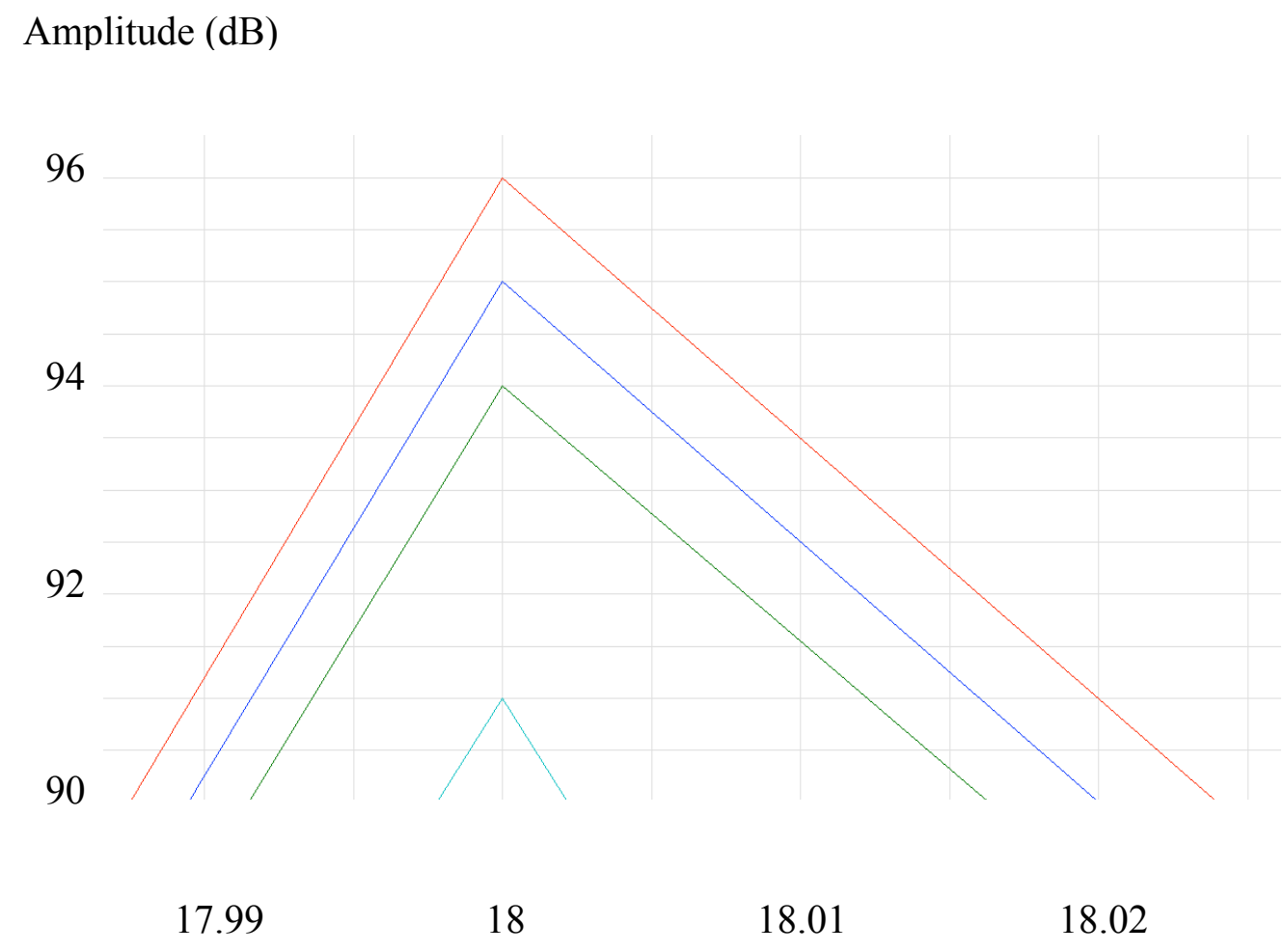

Time (s)

Figure 6: Expanded amplitude peak, second event from figure 5

\subsubsection{Corner Test}

In the following test pencil lead is broken four times, one time next to each of the four sensors used for triangulation. The results are seen in figure 7 on the following page. The hits again register slightly left of the predicted locations and a bit vertically above center as well. This can be attributed to both the error associated with the accuracy 
of the lead breaks, the error between the actual positions of the sensors and their virtual configuration in the AEWin software.

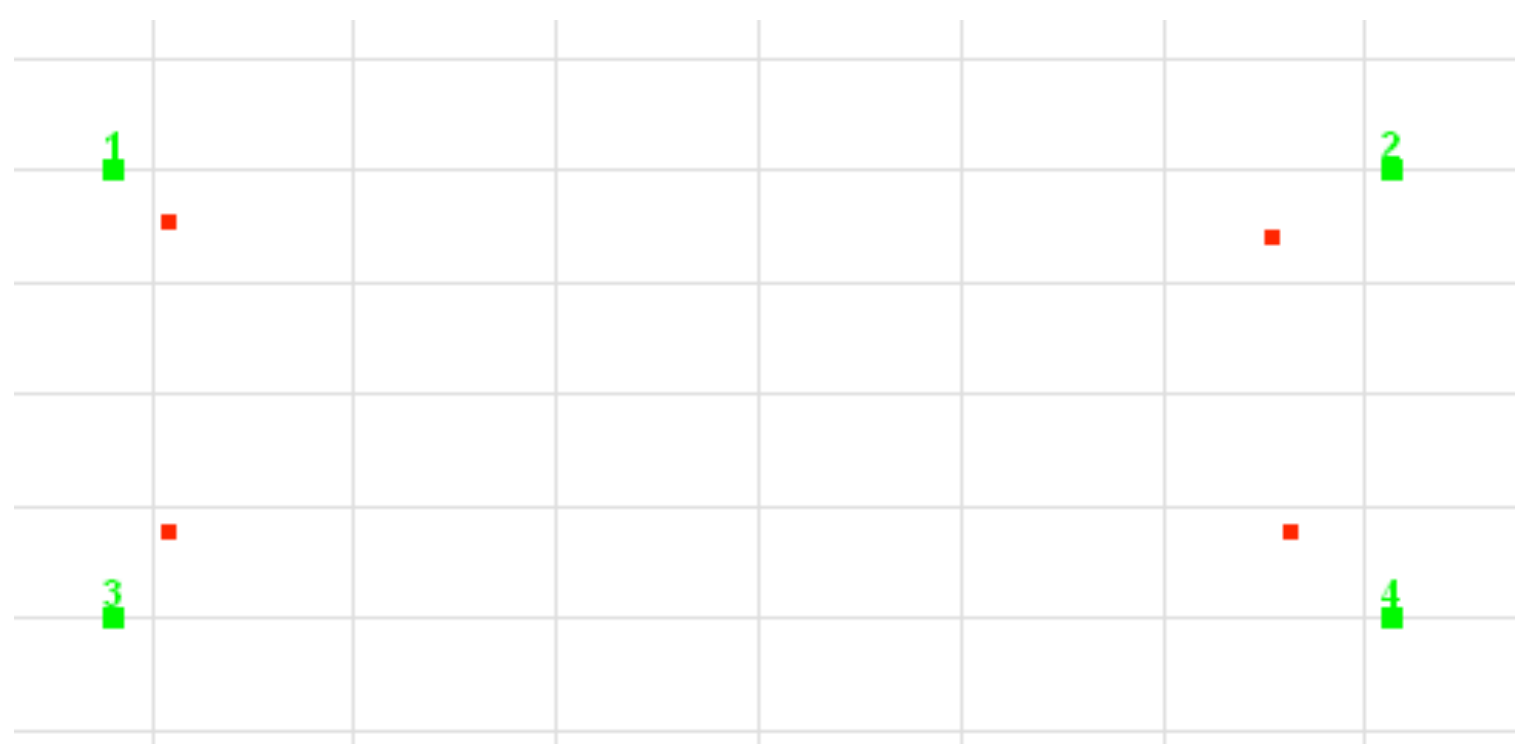

Figure 7: Four lead breaks in rectangular formation near the sensors

\subsection{Pencil Lead Test on Water-cooled Waveguide}

As an additional test, a lead is broken on the brass head of A water-cooled copper pipe waveguide (figure 8, next page) such waveguides are used in high temperature testing of materials. The waveguide is tested in conjunction with the running water through the guide while two sensors register events at its tail. The purpose of this test is to gain an understanding of how the acquisition will behave in the University of Vermont Inductively-Coupled Plasma Torch Laboratory. The waveguide has to be water-cooled to withstand the high temperatures. Thus, the sensors will not only monitor the excited 
sample at the head of the guide, but also monitor the water through the guide. More details on the construction of this waveguide appear in Chapter 3.

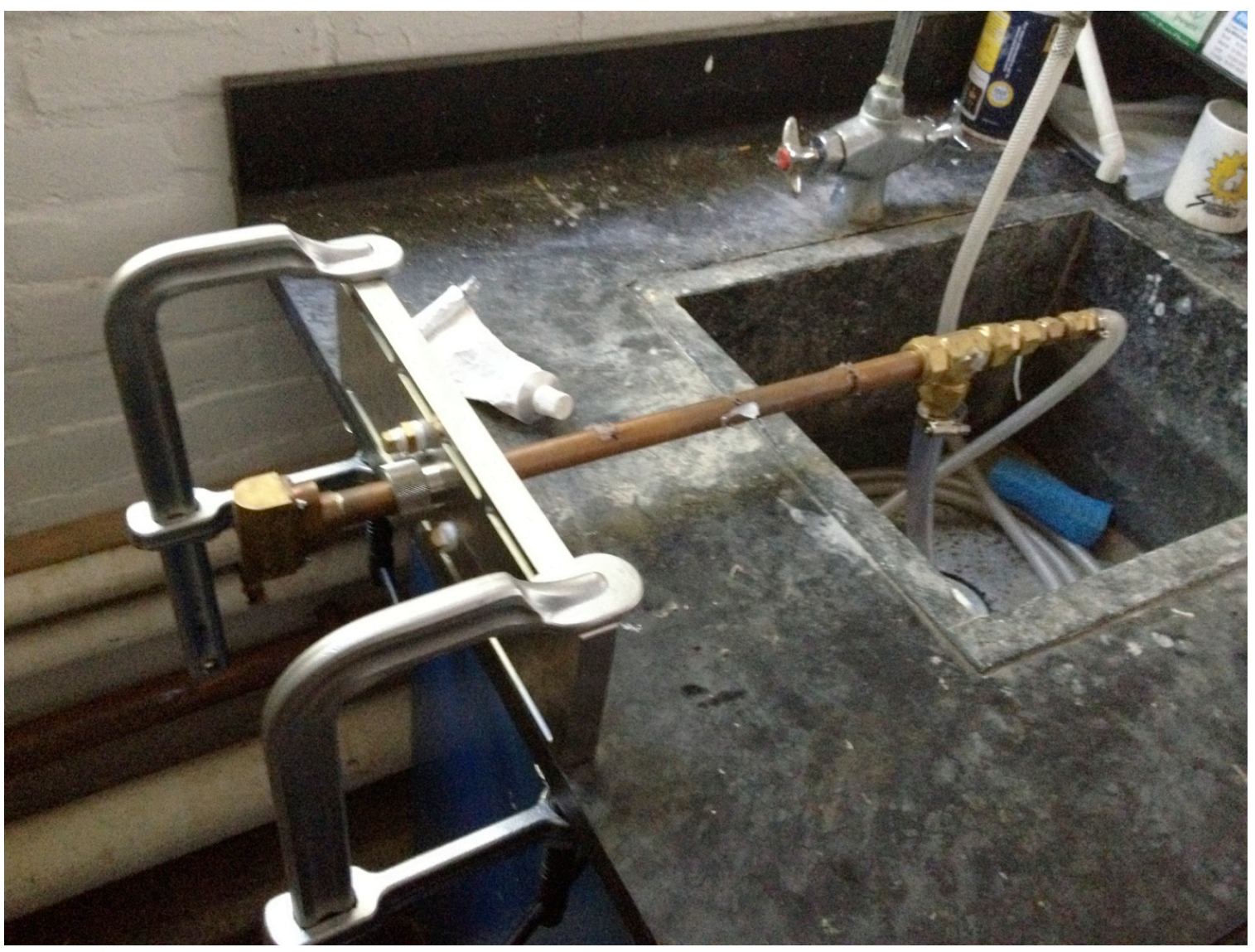

Figure 8: The water-cooled waveguide firmly clamped to a granite counter with its water intake connected to a faucet and an "exhaust" tube down the drain 


\subsubsection{Section Results}

Amplitude (dB)

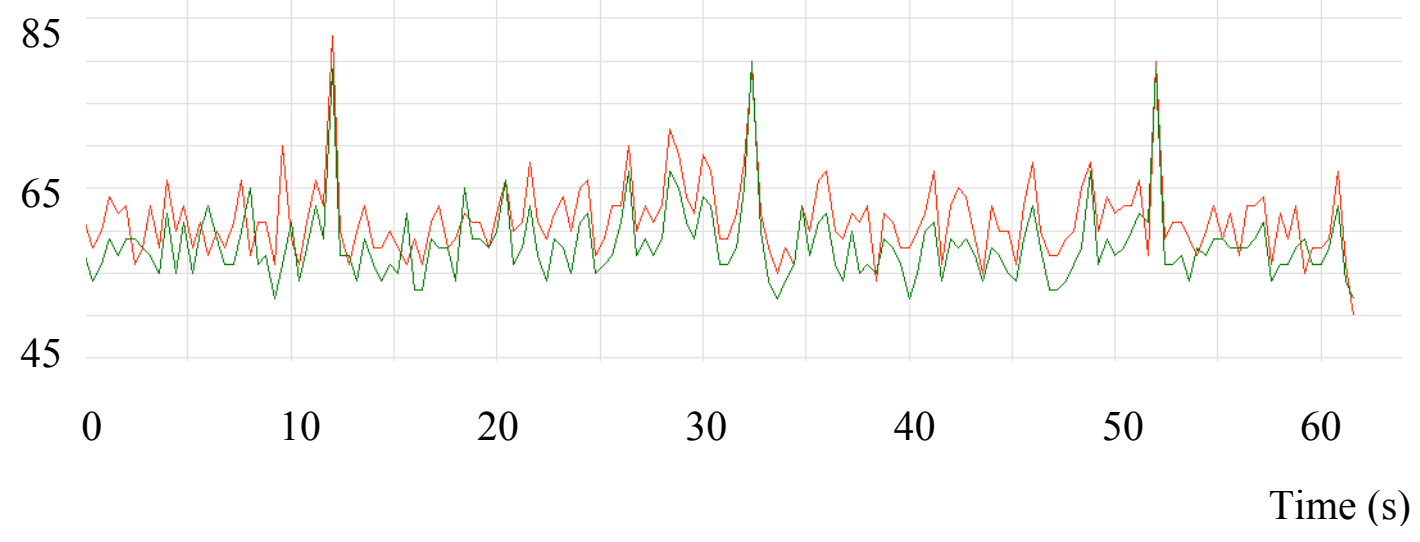

Figure 9: Two-sensor amplitude vs. time data, lead test on the water-cooled waveguide with running water

The pencil lead is broken in ten second intervals for a total of five breaks across a minute. The three trials at 10, 30, and 50 seconds clearly register with the sensors (the 3 peaks in figure 9), while the trials at 20 and 40 are harder to distinguish. The arrow in figure 9 likely indicates the break at 20 seconds; where the two sensors simultaneously both register similar amplitudes possibly indicates a greater event than the disarray associated with the water flow at time equal to 20 and 46 . The break at 40 seconds appears to be even less apparent, possibly related to the two steep peaks following the trough at 40 seconds; this could be due to a feeble break, weak lead, and/or an incorrect break angle; or perhaps the water flow is confounding the measurements by introducing large amounts of spurious acoustic noise or by more subtle fluid-elastic interactions that dampen the acoustic emissions of the pencil lead breaks. 


\section{CHAPTER THREE: LINEAR WAVEGUIDE EXPERIMENTS}

\subsection{Water-cooled Waveguide Experiment}

The water-cooled waveguide is designed to simulate the water-cooled waveguide in the plasma facility. Water runs at various flow rates through the waveguide with sensors placed at different locations for monitoring the associated acoustic emissions of the systems. The four sensors are placed in a variety of formations across several trials: two on either side of the brass T-connector, an additional two placed on either side of the brass housing comprising the waveguides head, four spaced evenly along the copper pipe (figure 10, next page), and three spaced evenly along the copper pipe (similar to figure 10) with one sensor on the T-connector [3].

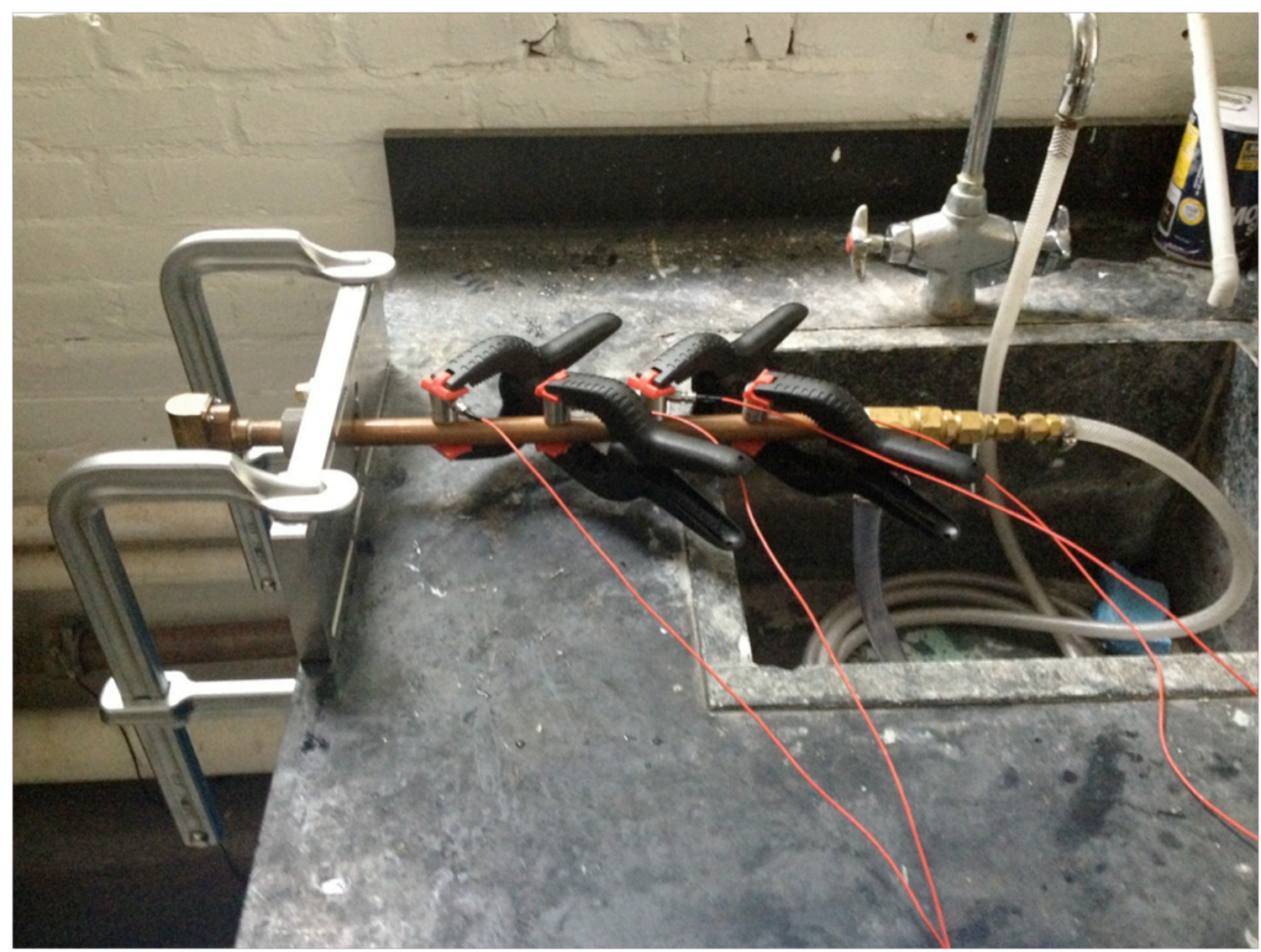

Figure 10: Four sensors spaced evenly along the copper pipe of water-cooled waveguide 
Inside the outer copper tubing of the waveguide is a smaller copper tube: water flows from the faucet, through an opaque hose, past brass connections, and down the smaller tube to the head of the waveguide. The flow is then redirected by an angled spout in the brass housing at its head, and sent back down the outer copper tubing, contained by the brass fixture.

Four flow rates are tested in rotation, five times each for approximately 20 seconds a trial (the water's flow rate is estimated before each trial. Thus, the five trials within each of the four categorical divisions represent similar flows, but not of precisely the same conditions). Twenty acquisitions from an adjacent two sensor T-connection configuration (figure 11) are converted into ASCII data and are interpreted with MATLAB.

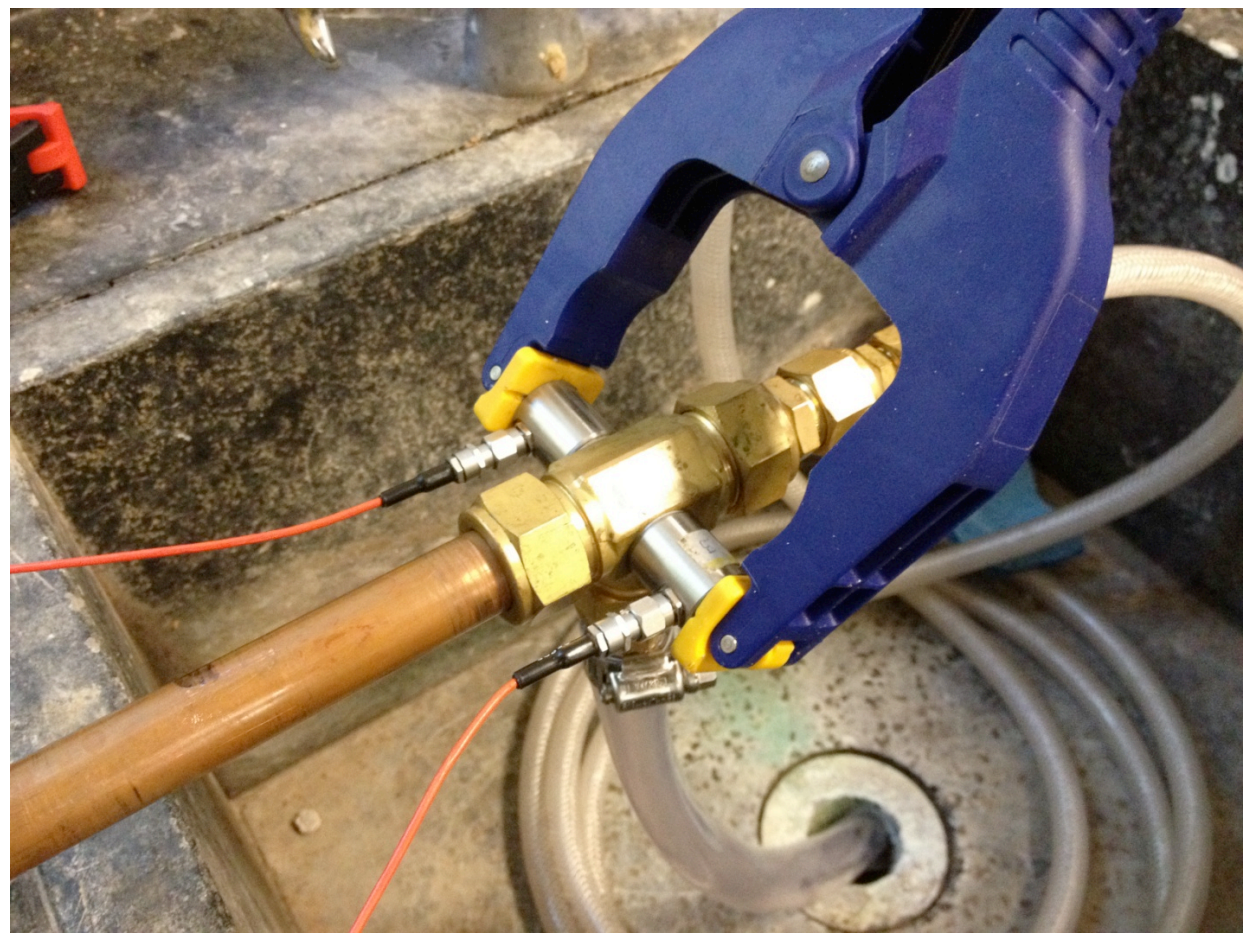

Figure 11: Two-sensor adjacent T-connection configuration, similar to the configuration that will be monitoring the waveguide in the plasma facility 


\subsubsection{Section Results}

The data are discriminated into four groups based on the median values of five characteristics (figure 14, page 21): energy, duration, average signal level, signal strength, and center frequency (detailed descriptions in table 1). The data are presented in glyph plot formations, arranged into visual groups defined by table 3 (page 22) with one spoke per variable from table 1 in a radial configuration (figure 12, next page). The trials are grouped using a multivariate measure of variance for the median value data in respect to the other trials [2]. 


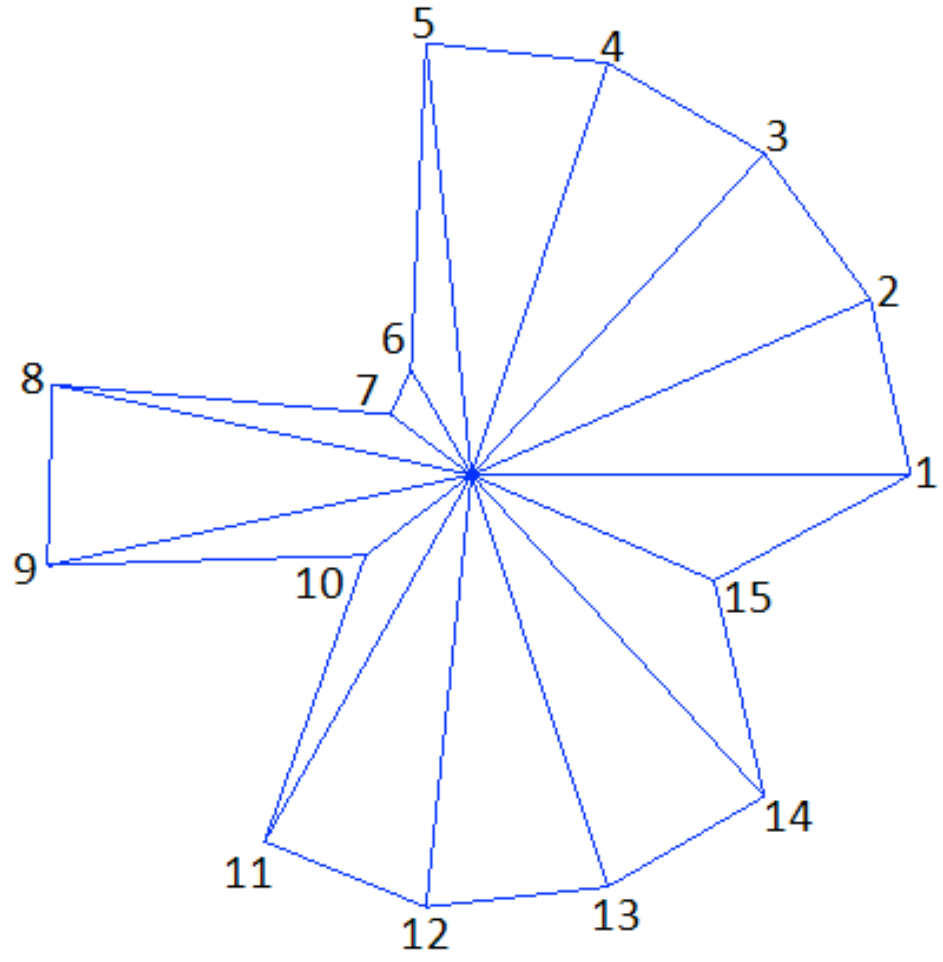

\begin{tabular}{|c|c|}
\hline Spoke & Feature \\
\hline 1 & Amplitude \\
\hline 2 & Duration \\
\hline 3 & Energy \\
\hline 4 & Counts \\
\hline 5 & Rise Time \\
\hline 6 & Peak Freq. \\
\hline 7 & Freq. Centroid \\
\hline 8 & Absolute Energy \\
\hline 9 & Signal Strength \\
\hline 10 & Initial Freq. \\
\hline 11 & Reverberation Freq. \\
\hline 12 & Counts-to-Peak \\
\hline 13 & Average Signal Length \\
\hline 14 & RMS \\
\hline 15 & Average Freq. \\
\hline
\end{tabular}

Figure 12: Glyph plot variable key [2] 


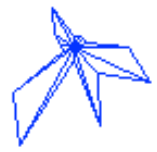

1

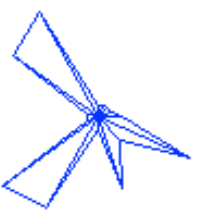

6

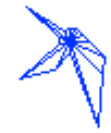

11

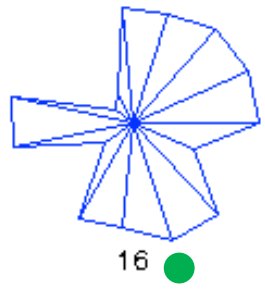

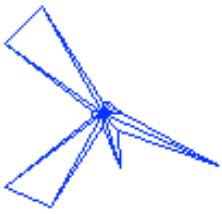

2

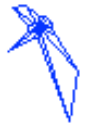

7

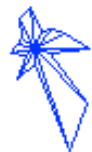

12

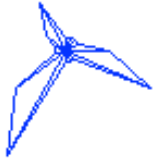

17

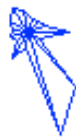

3

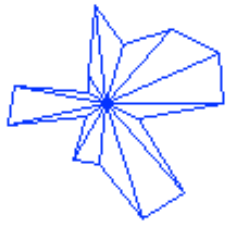

8

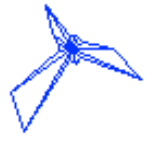

13

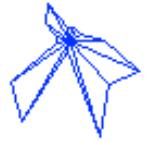

14

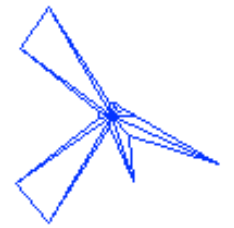

19

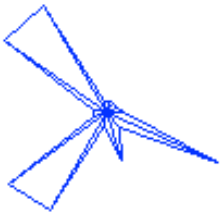

5

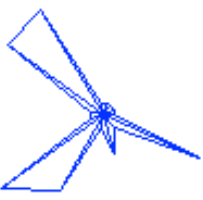

10

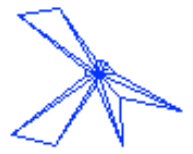

15

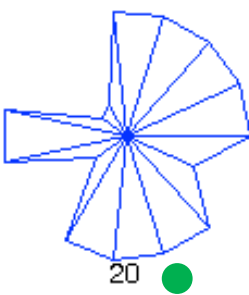

Figure 13: Glyph plots of the median data from the trials

Figure 13 depicts four different flow (table 2, next page) rates alternated after every fifth trial for twenty trials, alongside their corresponding Reynolds's Number. The Reynolds's number is a dimensionless quantity that characterizes flow. 


\begin{tabular}{|l|r|r|l|}
\hline Flow Rate & Reynold's Number & Categorical Label & Trial Numbers \\
\hline Trickle & 2.14 & & $1,5,9,13,17$ \\
\hline Slow & 33.3 & & $2,6,10,14,18$ \\
\hline Moderate & 66.6 & & $3,7,11,15,19$ \\
\hline Full & 114.9 & & $4,8,12,16,20$ \\
\hline
\end{tabular}

Table 2: Trial numbers, corresponding flow rates, and color labels per flow category

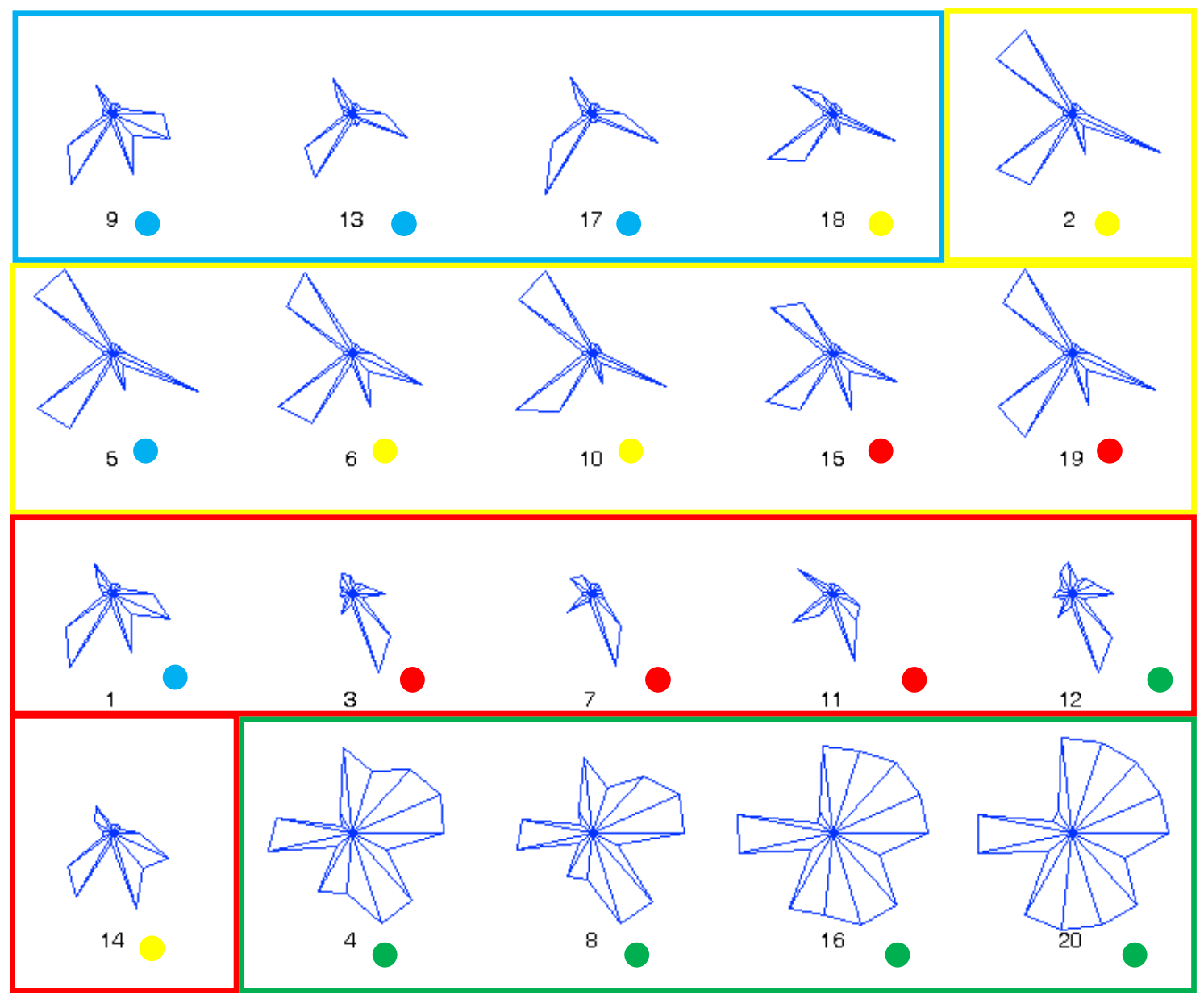


Figure 14: Grouped glyph plots, clustered by the color representing the most prevalent flow rate of the group, numerical and categorical labels carried from previous figure

\begin{tabular}{|c|c|c|c|}
\hline Group & Cluster Identifier & Trial Numbers & Most Frequent Flow Rate \\
\hline 1 & $\Gamma$ & $9,13,17,18$ & Trickle \\
\hline 2 & & $2,5,6,10,15,19$ & Slow \\
\hline 3 & & $1,3,7,11,12,14$ & Moderate \\
\hline 4 & & $4,8,16,20$ & Full Speed \\
\hline
\end{tabular}

Table 3: Four discriminated groups of trials with the trials most frequent flow rate

\subsubsection{Linear Waveguide Testing Conclusion}

The classification of the flow rates is considered a success. The "misclassified" trials (trials with a different color categorical label from their cluster identifier color) can be attributed to estimates and control of the different flow rates across the twenty trials (the rates were estimated by matching audible noise emitted from the faucet per setting, and confirmed with visual estimates from the "exhaust" of the flow, evaluating the flow against preceding trials). The extended periods of turbulence due to the flow could have possibly throw off the sensors from a constant position altering the results per trial. A more precise experiment could be to measure all similar flows simultaneously before altering the flow rate, include an actively monitored flow so the conditions repeat more precisely between trials, or a completely redesigned test-bed possibly including sensors embedded within the waveguide. 
Groups one, two, and three exhibit higher frequency data while group 4 Group demonstrates higher energy and signal data. For this reason group four is most distinct (energy and signal level are two of the five classification features) with four correctly classified trials, only failing trial 12 (misclassified by one group as a moderate flow with lesser energy and a shorter duration from the trials in group 4). Trial one is the greatest outlier of this experiment: the first trickle flow trial is misclassified as a moderate flow, which is two groups away from the appropriate experimental category.

The purpose of these experimental trial is twofold: to classify AE data based upon a categorical variable or grouping; to analyze an acoustical event occurring over the "noise" of the running water of the water-cooled waveguide.

\subsection{Uncooled Waveguide Experiments}

Two separate experiments are conducted with waveguide 2 (figure 15): an actuated graphite sample (seen in figure 16, next page) piezoelectric excitation test and a butane torch cork burn test [2].

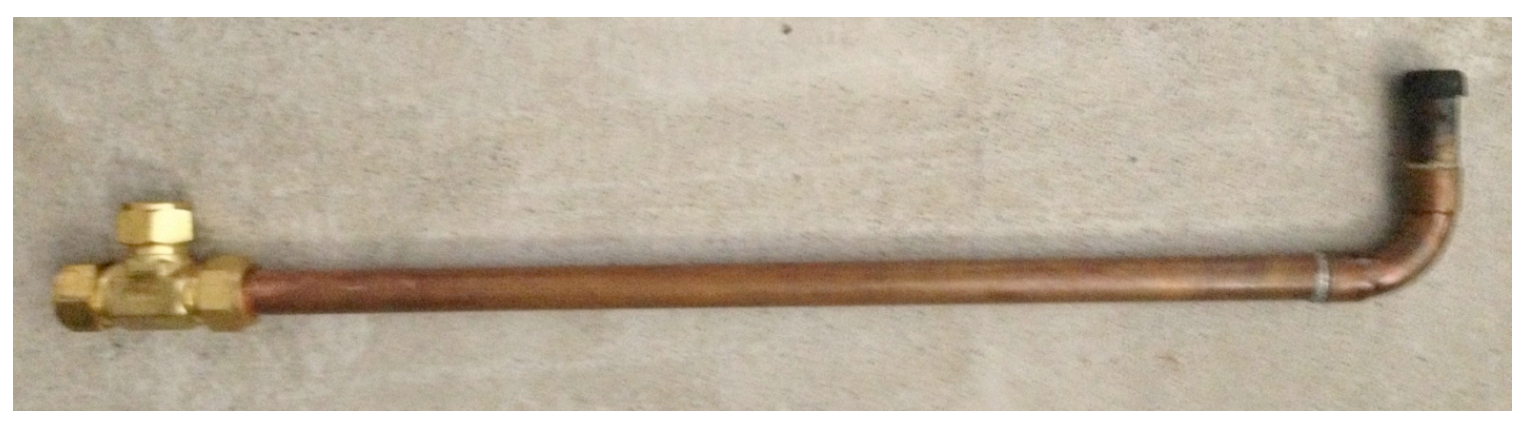

Figure 15: Waveguide 2 with a graphite sample in the holder, right 
An R15 $\alpha$ sensor is inverted for actuation by running an amplified signal through the sensor's BNC connection. The sensor is placed directly against the graphite sample face and held firmly in place (figure 18, page 25). A function generator is programmed to sweep a linear chirp signal (a sine wave with a linearly increasing frequency) from 50 $\mathrm{kHz}$ to $550 \mathrm{kHz}$ over a period of a half second following an external trigger. Its output is split and linearly amplified: a scaled up version to power the inverted sensor and a second scaled version fed to the AEWin software through a PAC preamp ( $20 \mathrm{~dB}$ setting) as seen in figure 17 on the following page. A second sensor is placed on the brass T-connection of the waveguide for AE monitoring (to the right in figure 19, page 26). The TTL output of a second function generator is split, capable of simultaneously triggering both the chirp waveform and the software acquisition. The software is programmed to acquire data across several periods of one second, matching the period of the chirp waveform; the acquisition is triggered and stopped by a user [2].

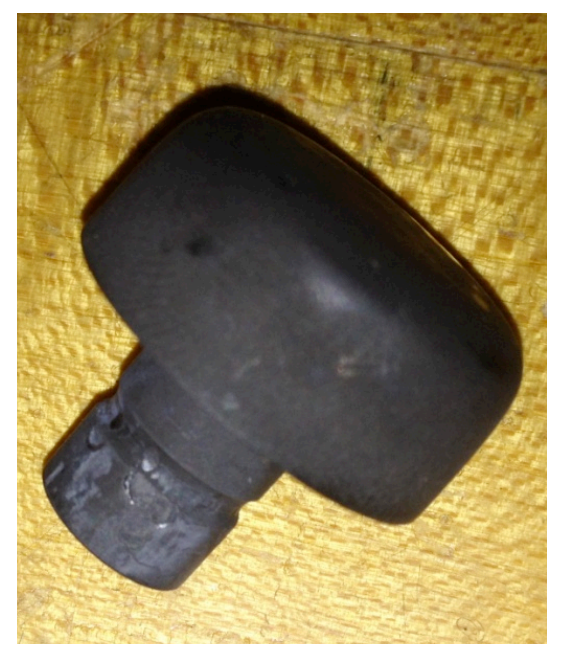

Figure 16: The graphite sample from figure 15 out of the holder 


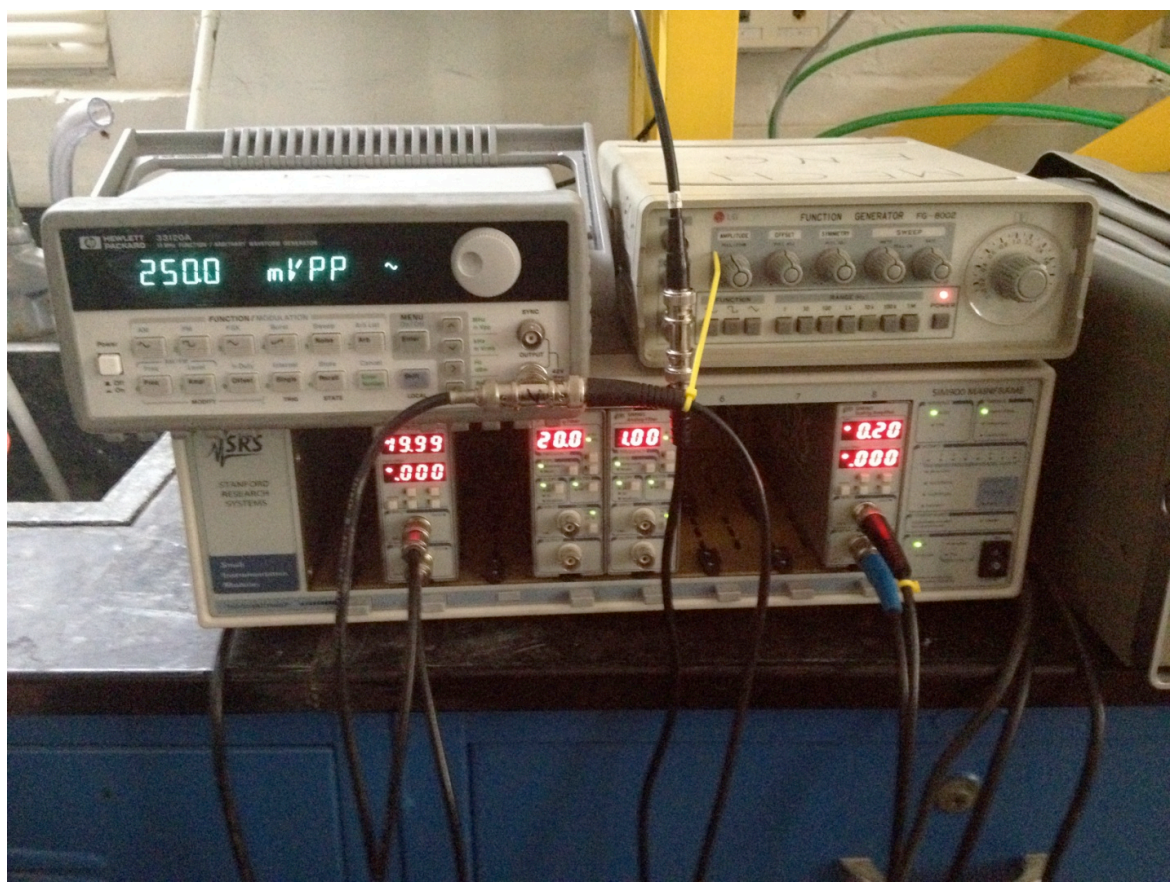

Figure 17: Two function generators on a housing with four linear amplifiers; top left is the chirp waveform generator; top right is the TTL trigger; bottom outside modules in the housing are the units used for the linear scaling of the waveform

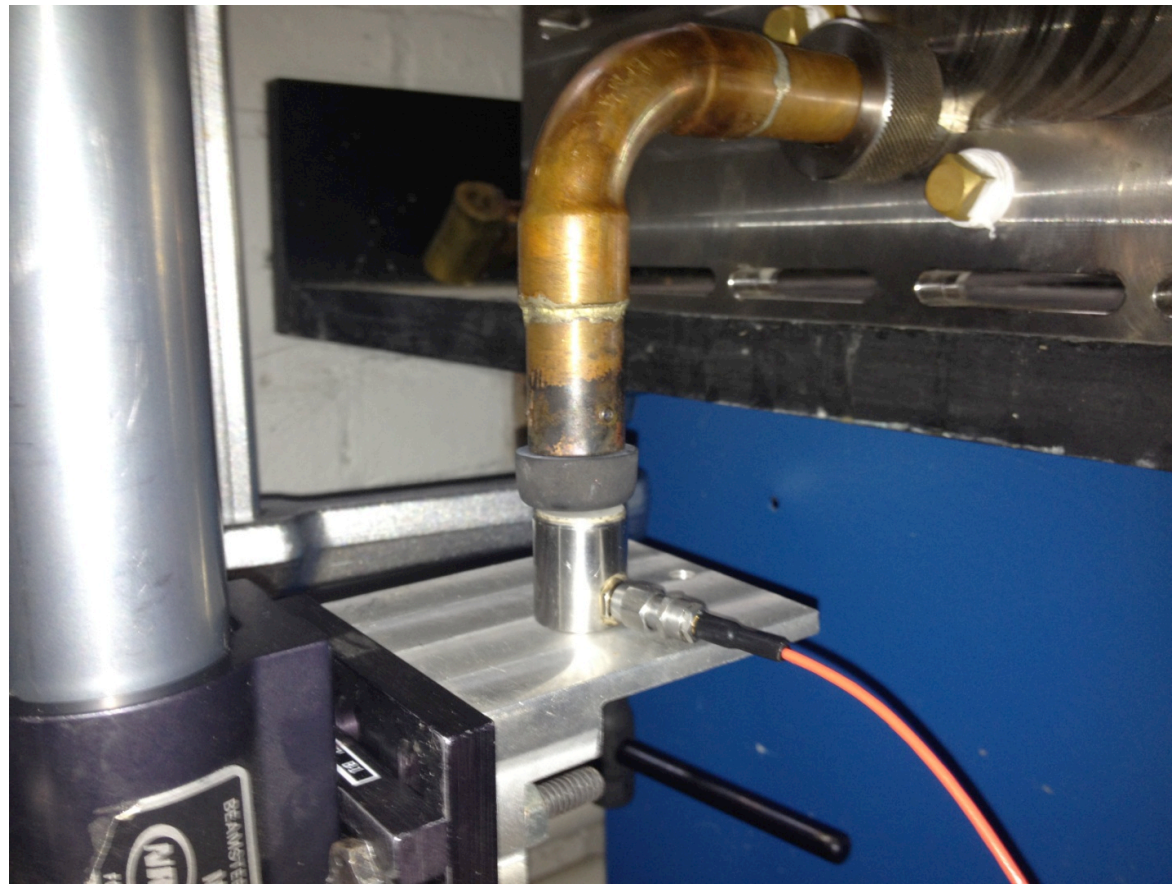

Figure 18: Actuated sensors placed firmly against the graphite sample 


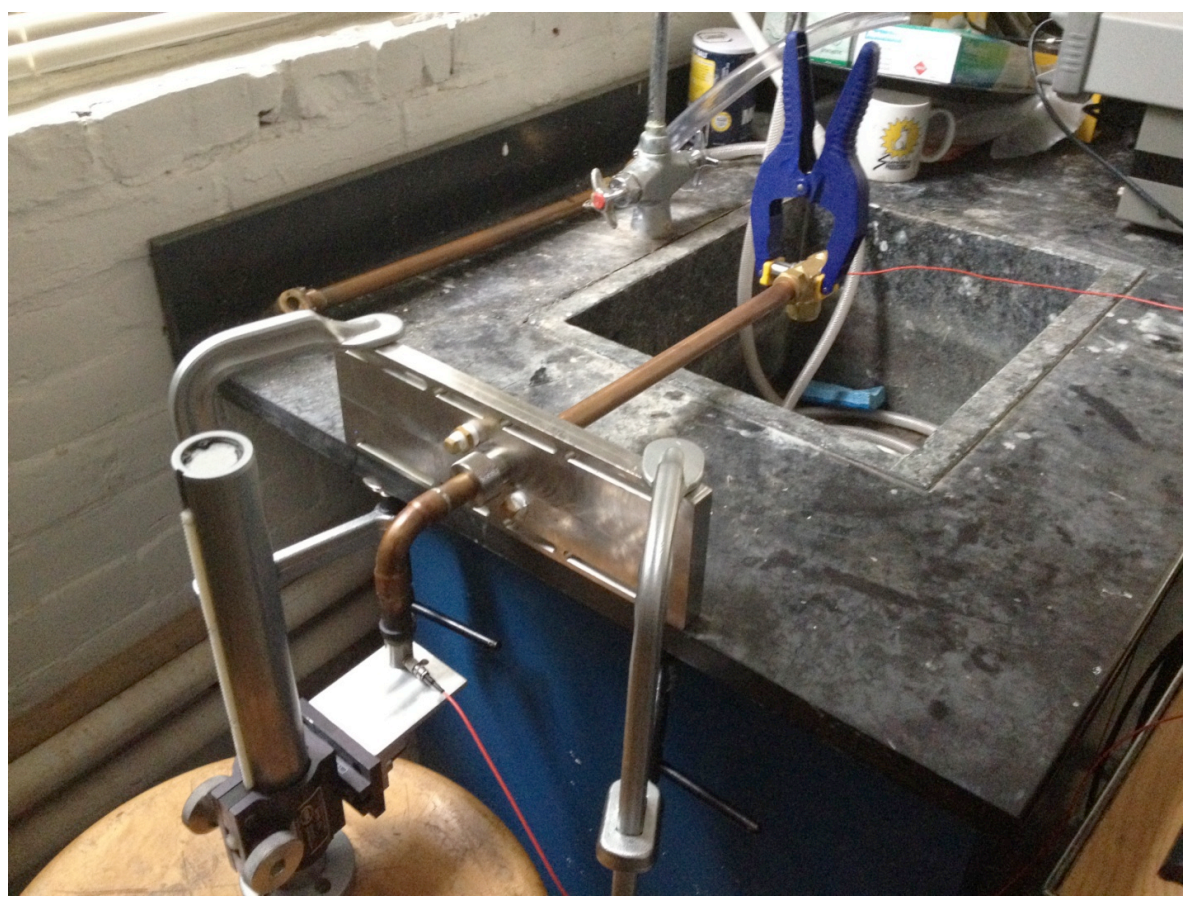

Figure 19: Both sensors shown

\subsubsection{Results}
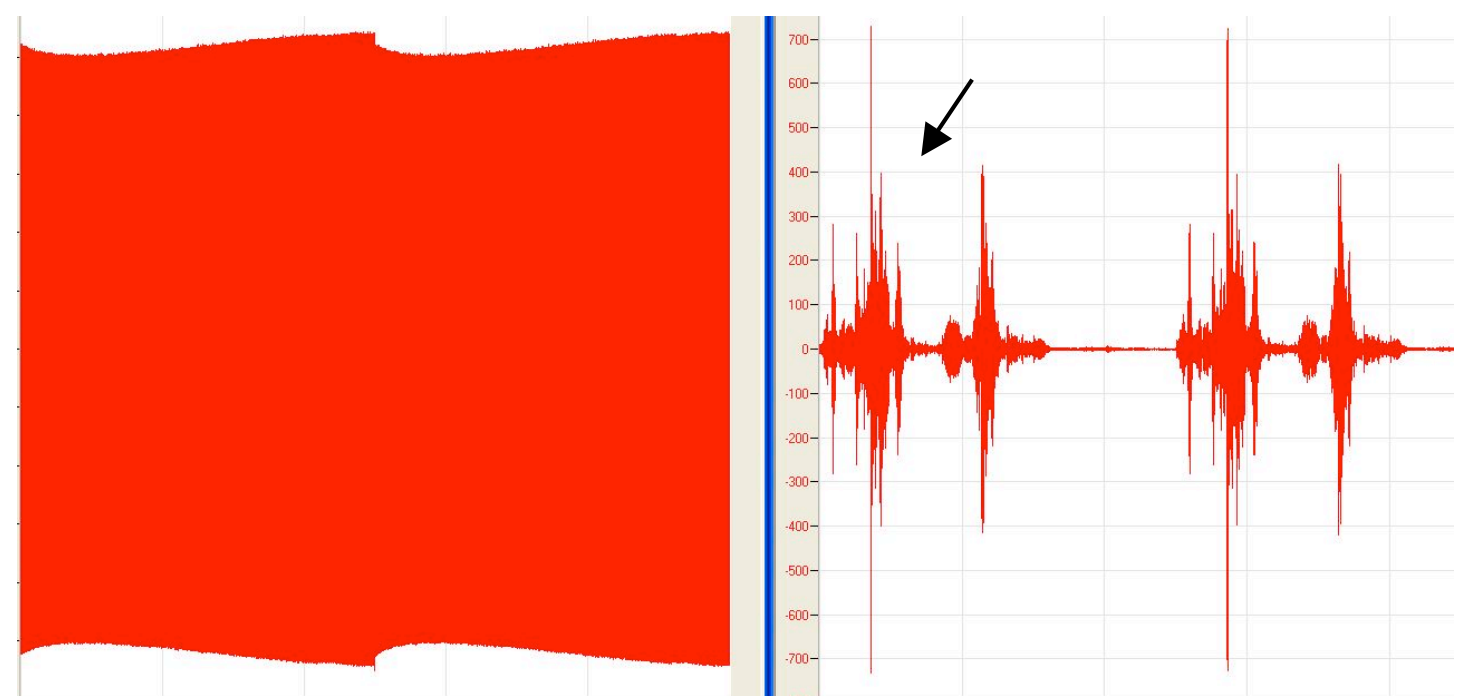

Figure 20: Left, the scaled chirp-waveform cycling twice in one second; right, the resulting AE signal from the second sensor 


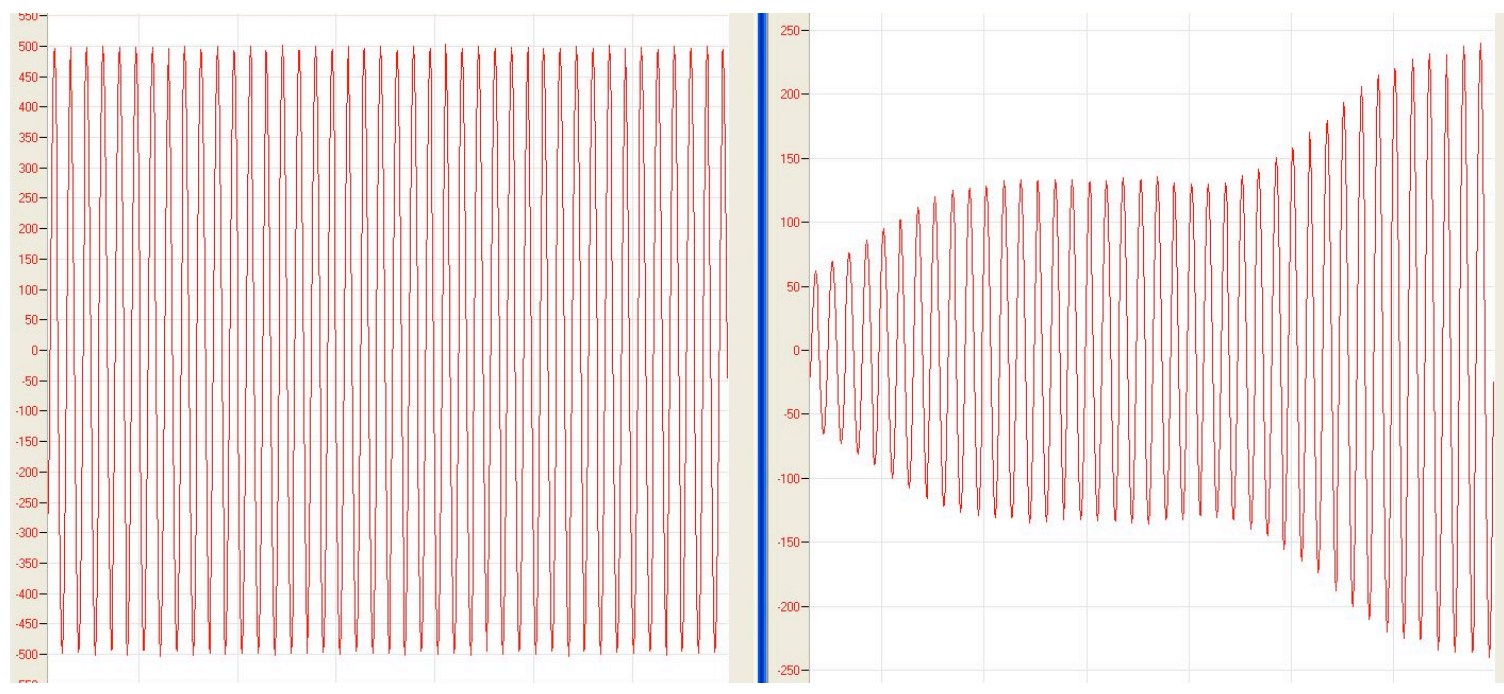

Figure 21: Zoomed in versions of the waveforms, in the excited area directed by the arrow in figure 20

The voltage from the chirp waveform from figure 20 generator does not remain entirely constant; it fluctuates between approximately $500 \mathrm{mV}$ to $550 \mathrm{mV}$, amplified at around twice the output of the chirp waveform generator. It is clear in figure 20 that the waveguide experiences multiple periods of excitation across the cycle, with the largest pulse ramped up from the beginning of the sweep indicated by the arrow (zoomed in on in figure 21). A similar excitation follows at about a fifth of the way through the cycle and of slightly lesser magnitude. The waveguide's response to the excitation at the sample head becomes weaker past half way through the cycle; at higher frequencies of actuation no sort of significant resonance is present. The second cycle yields quite similar response to the first. 


\subsection{Butane Torch Excitation Experiment - Uncooled Waveguide}

The cork from a Champagne bottle is whittled down to fit into the sample holder and fixed firmly to the uncooled waveguide. The waveguide is positioned under a hood to collect the exhaust from the experiment seen in figure 22, next page. A butane torch is held with a stand directly over the cork; two sensors are applied to the T-connection of the uncooled waveguide (figure 23, page 30). The test is initiated: the torch is fired in hands-free mode, roasting the cork across a period of over a minute (figure 24, page 30); the test is rerun. 


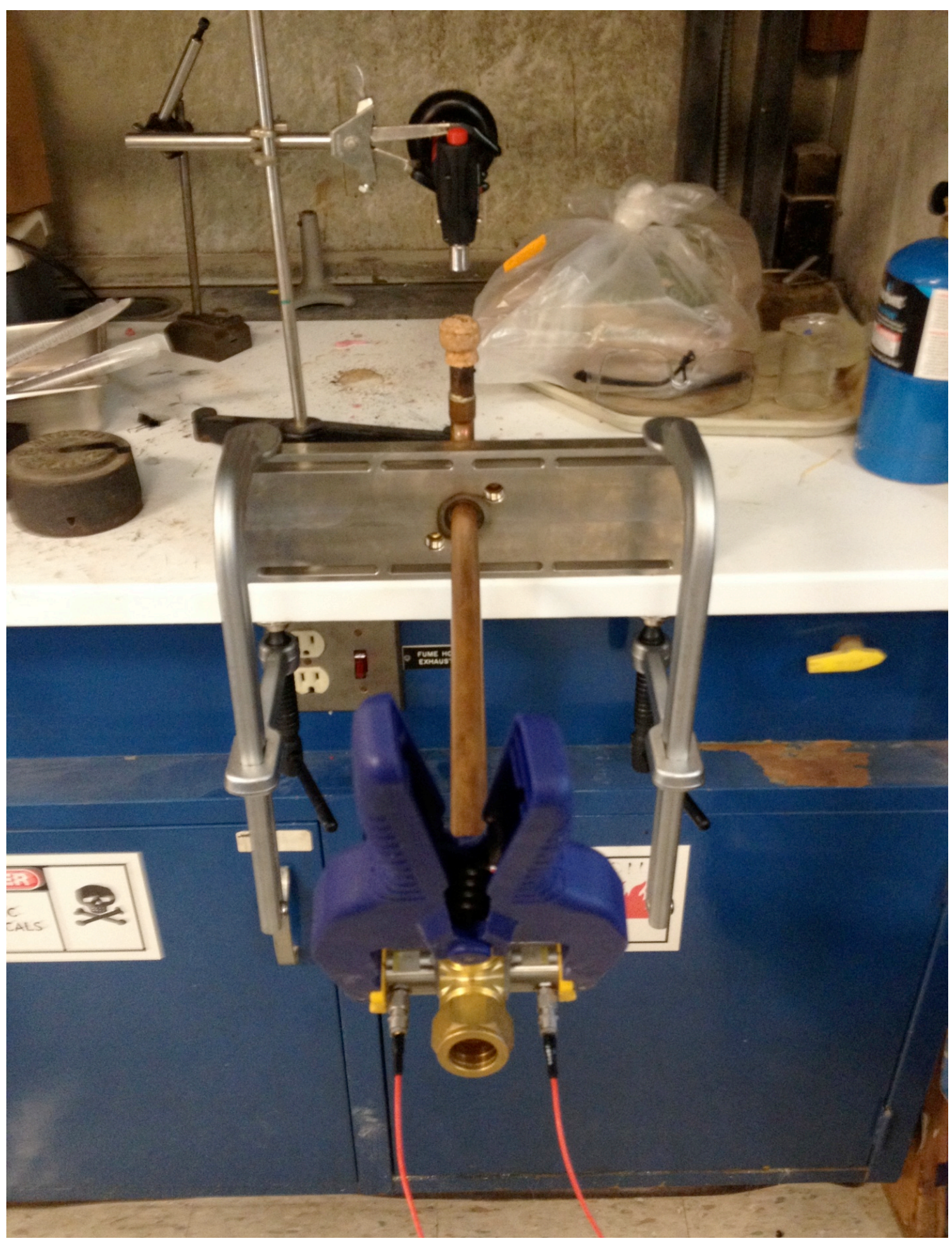

Figure 22: View down the uncooled waveguide, butane flame test 


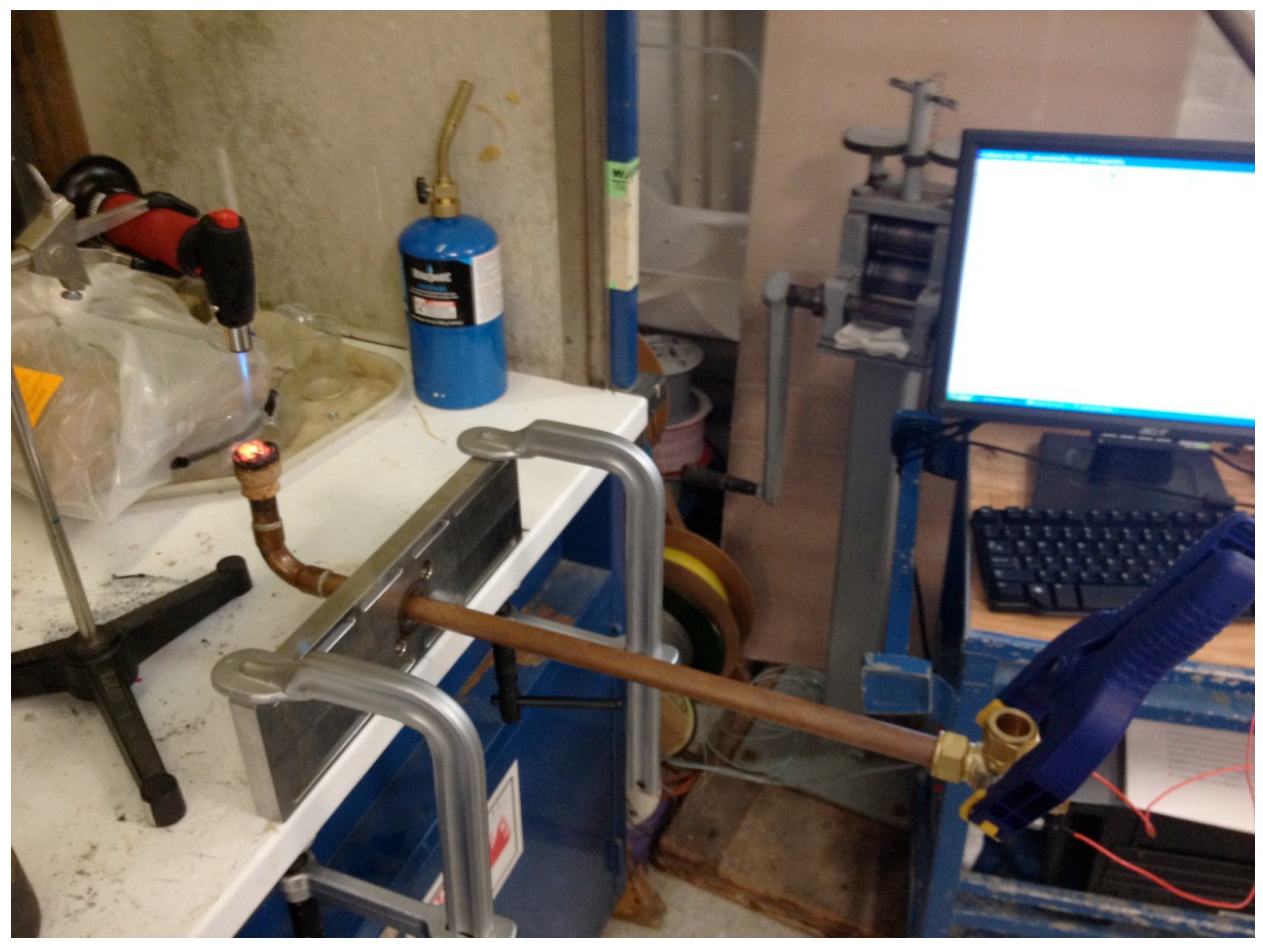

Figure 23: The butane torch is applied to cork sample while AEWin is collecting data

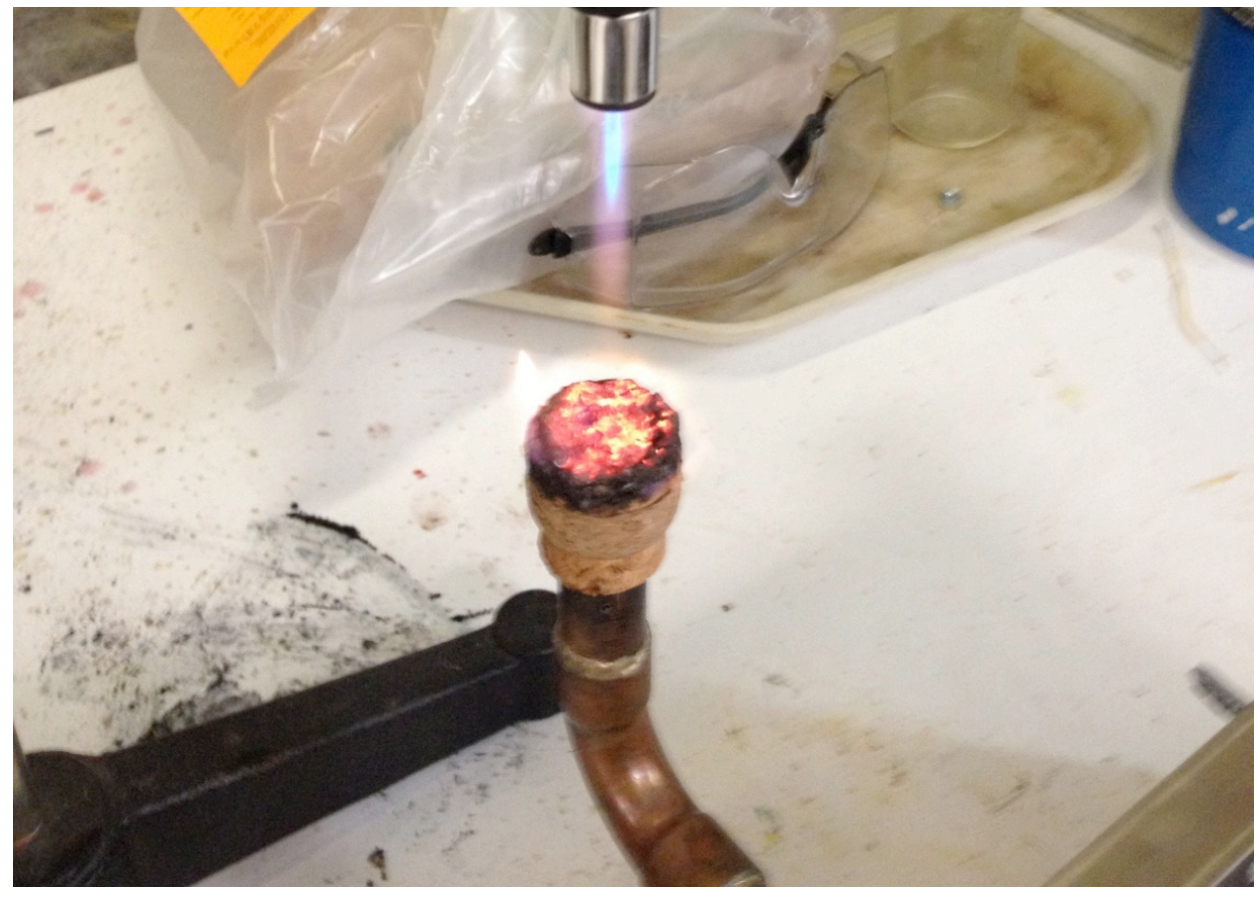

Figure 24: Close up of the burning cork sample 


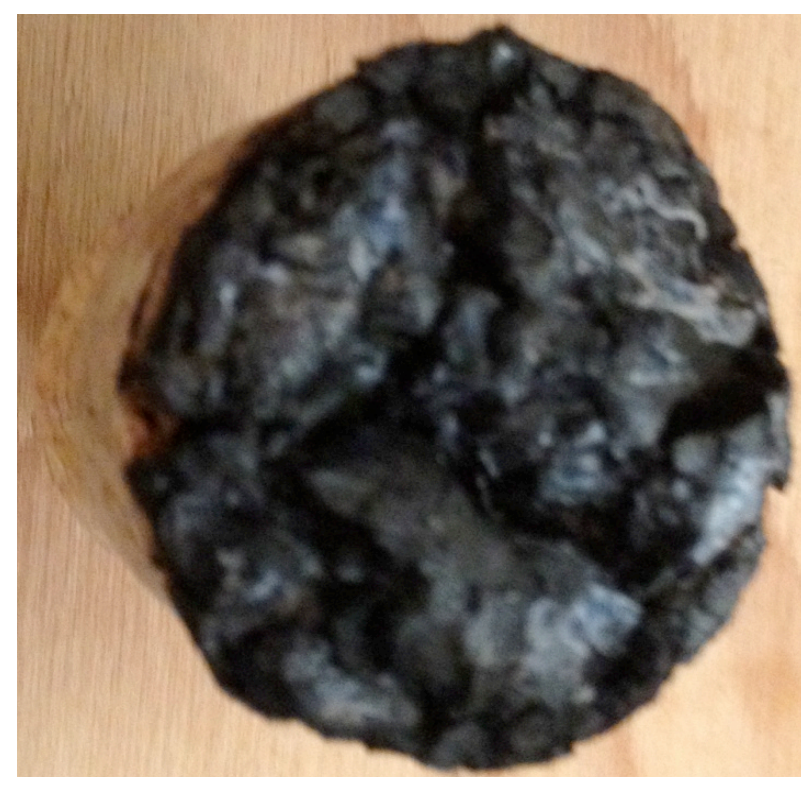

Figure 25: Charred cork

\subsubsection{Section Results}

A carbon based charred face remains, hardened by the flames of the torch (figure 25). Data are collected across seven trials. Unfortunately, it seems the torch is barely powerful enough to trigger the threshold voltage of the software; events are registered but only directly following torch ignition, possibly due to an initial impulse, or a fresher cork face. It was hard to get the torch to stay lit; the trial below represents hits from two ignitions of the torch, with the torch remaining lit for around only a second. 

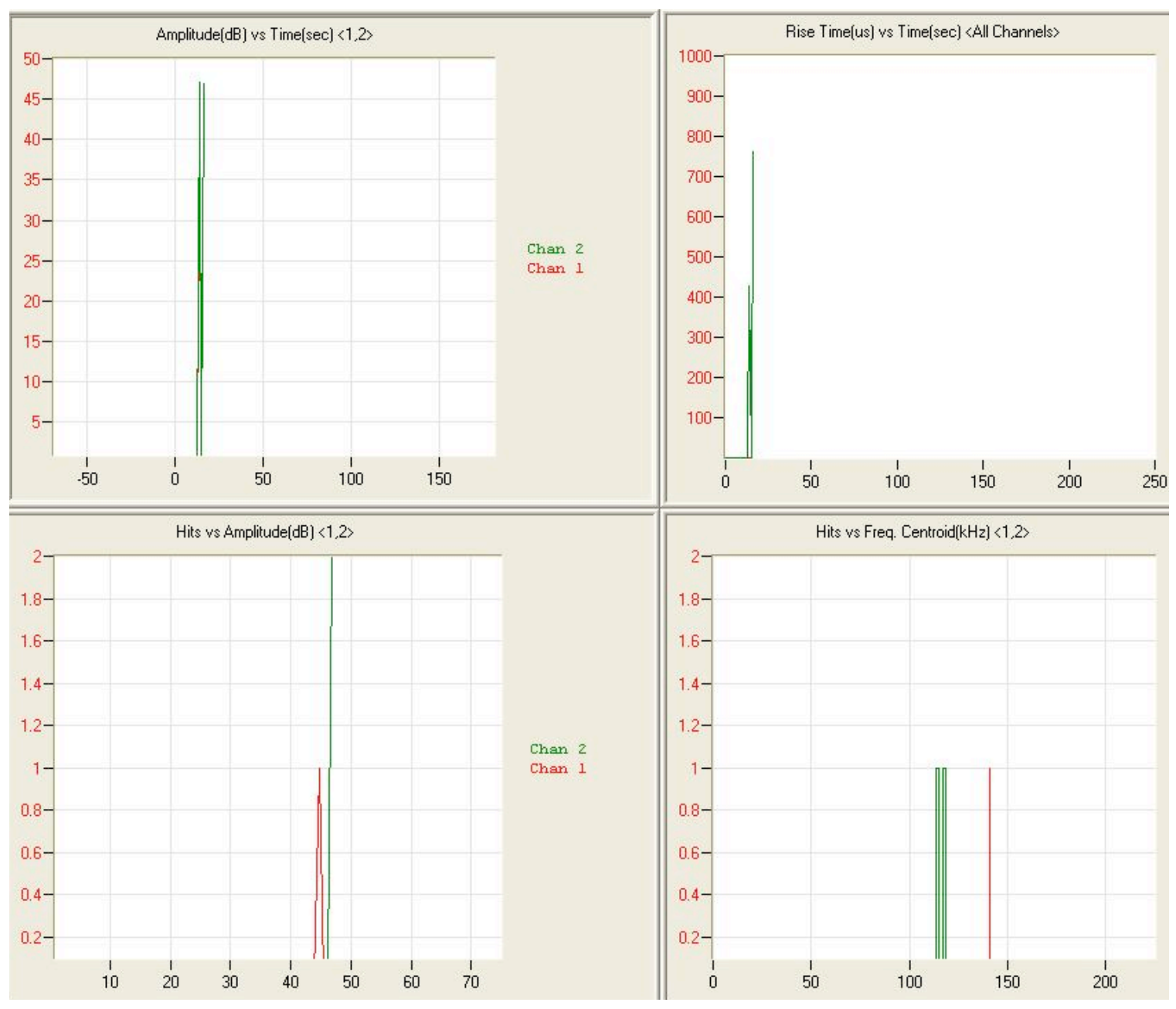

Figure 26: AE data from two ignitions of the butane torch, held directly overhead of the sample

The three hits collected from the two ignitions in figure 26 provide further evidence that the torch is too weak to meet the threshold voltage since only one of the sensors registers for one of the ignitions (the other sensor not surpassing the threshold): amplitude vs. time, rise time vs. time, hits vs. amplitude, and hits vs. frequency centroid. 


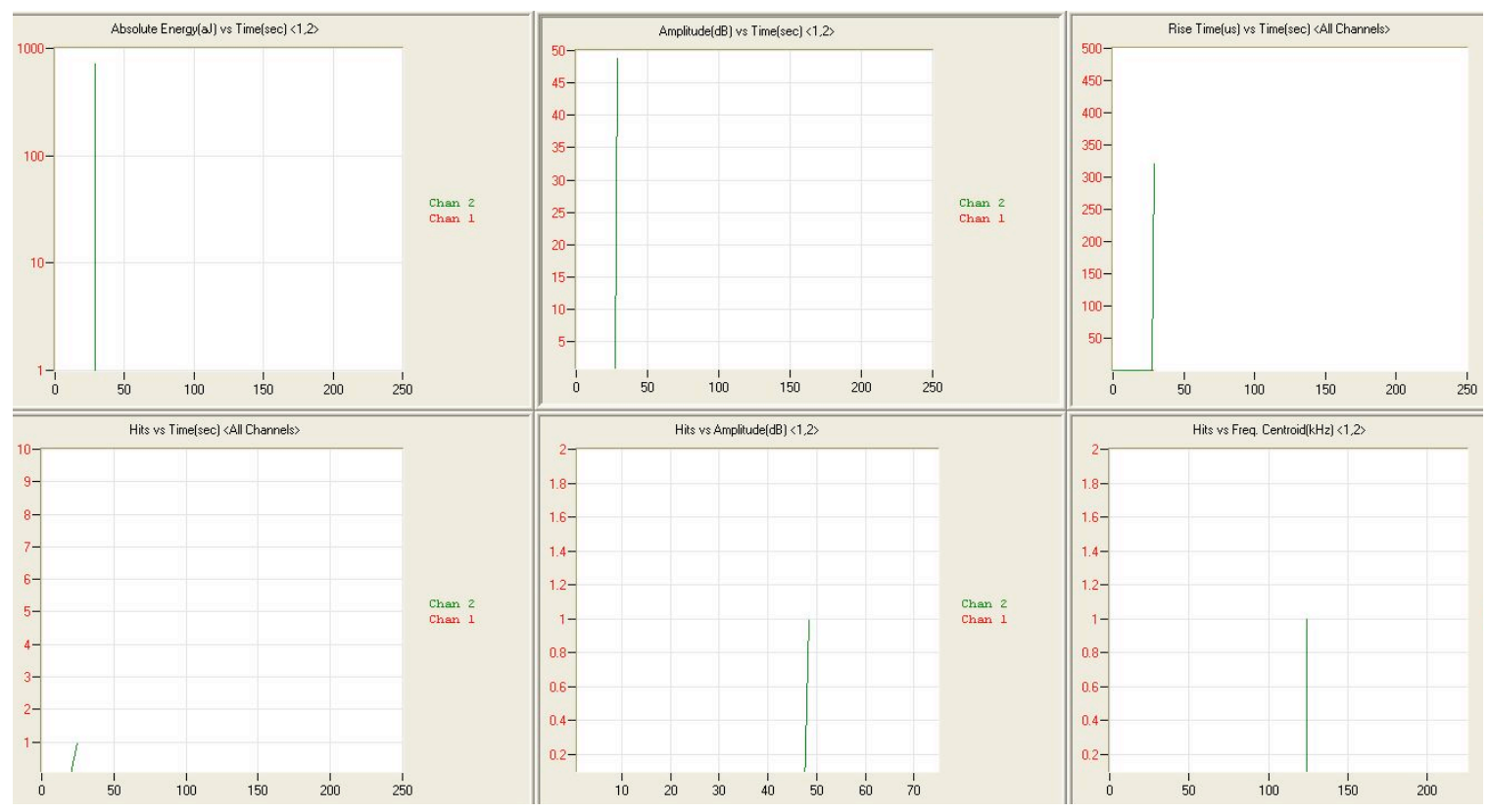

Figure 27: AE data from a trial where the torch is ignited and then moved over the head of the sample

Figure 27 shows one hit from a trial where the torch was ignited and then moved the torch overhead of the sample, after charring the sample several times across a period of two or three minutes; the hit registers following the placement of the torch over the sample.

\subsubsection{Conclusion of Butane Torch Excitation Experiment for the Uncooled}

\section{Waveguide}

The purpose of the butane torch test is to model something similar to the plasma torch waveguide interaction in the plasma lab facility. The test is partially successful and could be repeated with a stronger torch, and/or a lower threshold voltage. 
CHAPTER FOUR: INDUCTIVELY-COUPLED PLASMA TORCH LAB

\section{TESTING}

\subsection{Test Setup and Procedure}

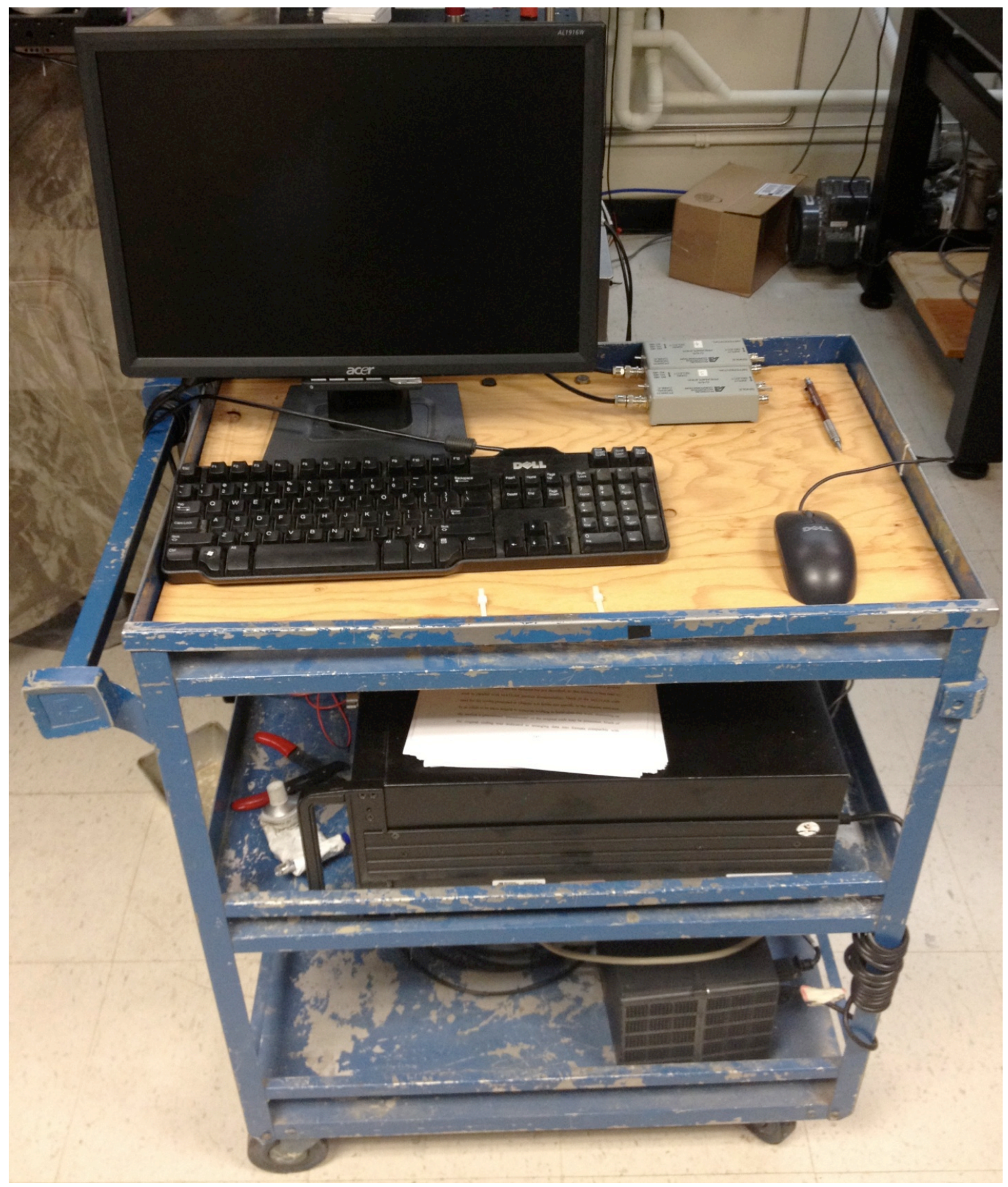

Figure 28: The data acquisiton cart 


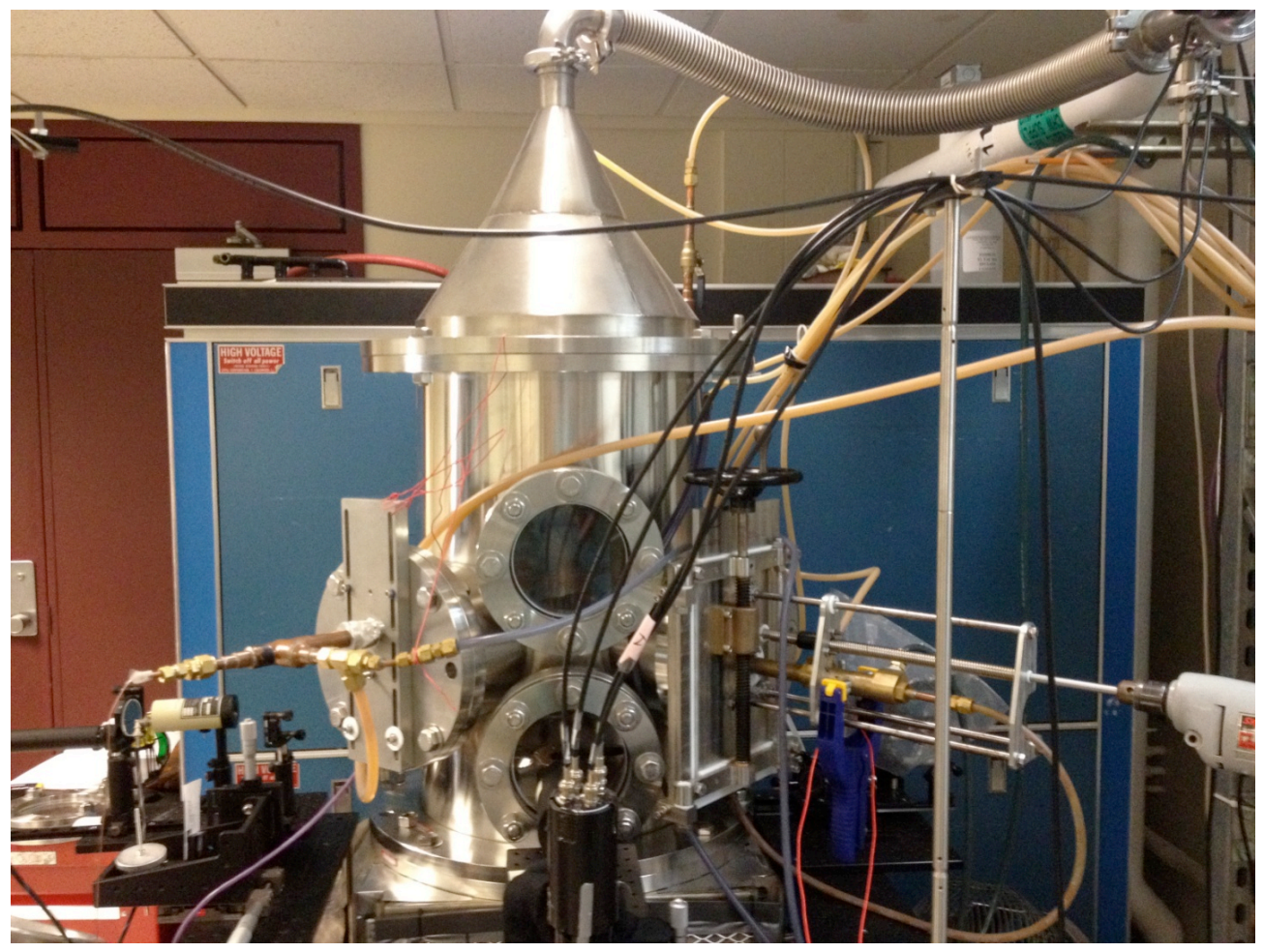

Figure 29: UVM's Inductively Coupled Plasma ICP Torch

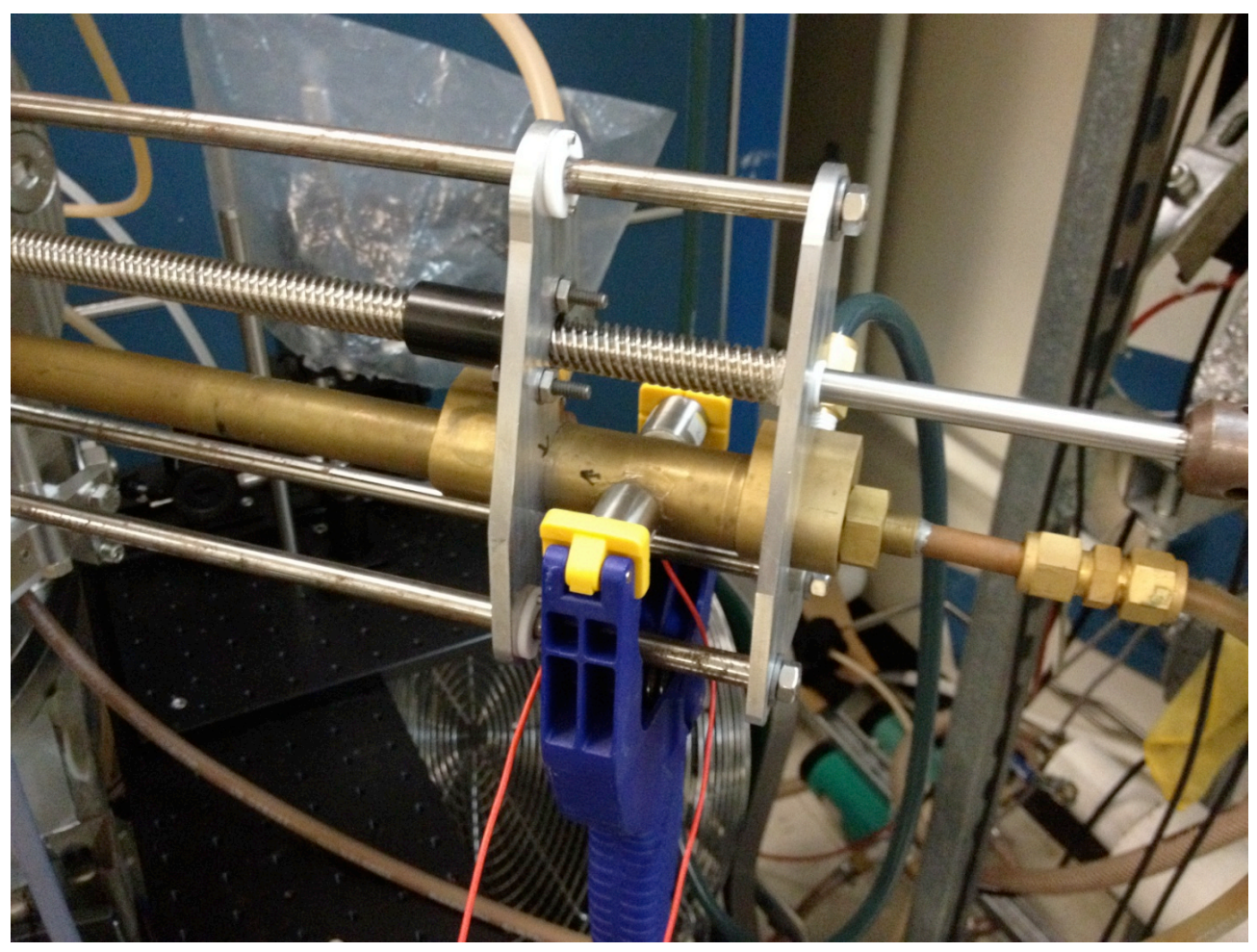

Figure 30: Close up of sensor Placement, brass water-cooled waveguide in the ICP torch facility 
A graphite sample is held by the sample holder inside the plasma torch at the head of the water-cooled waveguide. The waveguide can be seen bottom-right of figure 29 and in figure 30. Two sensors are placed on adjacent sides of the tail end of the waveguide (figure 30) and the facility is prepared for testing. The torch is brought up to pressure with a vacuum pump system followed by the argon, which is ignited at 120 Torr. The torch is then brought to the desired composition of gasses and the pressure is leveled at 160 Torr. The sample is inserted via waveguide with the drill seen far right in figure 29. Once inside the plasma stream the sample is left for a period of 5 minutes. Seven successful trials with a single graphite sample and multiple plasma compositions are conducted with AE data collected for the duration of each trial with the cart in figure 28 . Temperature data are also collected for the trials with initial and final sample mass collected per trial as well.

\subsection{Chapter Results}

\begin{tabular}{llllll}
\hline Trial & Plasma Composition (SLPM) & Initial Sample Mass & Final Mass & Mass Change & Loss Rate \\
\hline $\mathbf{1}$ & 10 Argon / 30 Nitrogen & 12.948 & 12.848 & 0.1 & 0.0003333 \\
$\mathbf{2}$ & 10 Argon / 30 Nitrogen & 12.848 & 12.763 & 0.085 & 0.0002833 \\
$\mathbf{3}$ & 10 Argon / 30 Nitrogen & 12.763 & 12.657 & 0.106 & 0.0003212 \\
$\mathbf{4}$ & 10 Argon / 30 Nitrogen & 12.657 & 12.563 & 0.094 & 0.0003481 \\
$\mathbf{5}$ & 40 Nitrogen & 12.943 & 12.838 & 0.105 & 0.0003500 \\
$\mathbf{6}$ & 30 Argon / 10 Nitrogen & 12.838 & 12.78 & 0.058 & 0.0001933 \\
$\mathbf{7}$ & 40 Argon & 12.78 & 12.766 & 0.014 & 0.0000467 \\
\hline
\end{tabular}

Table 4: Graphite sample test data, plasma torch 
From the data in table 4 is fairly clear that the graphite sample loses mass at a greater rate due to the nitrogen than the argon gas, with the greatest mass loss rate occurring during trial 5 with a hundred percent nitrogen composition. The AE data presented in figures $31-36$ on pages $37-40$ depicts 5 of the 15 features collected and calculated by the AEWin software. These features can be statistically analyzed across several acquisitions to help find trends and classify data [3].

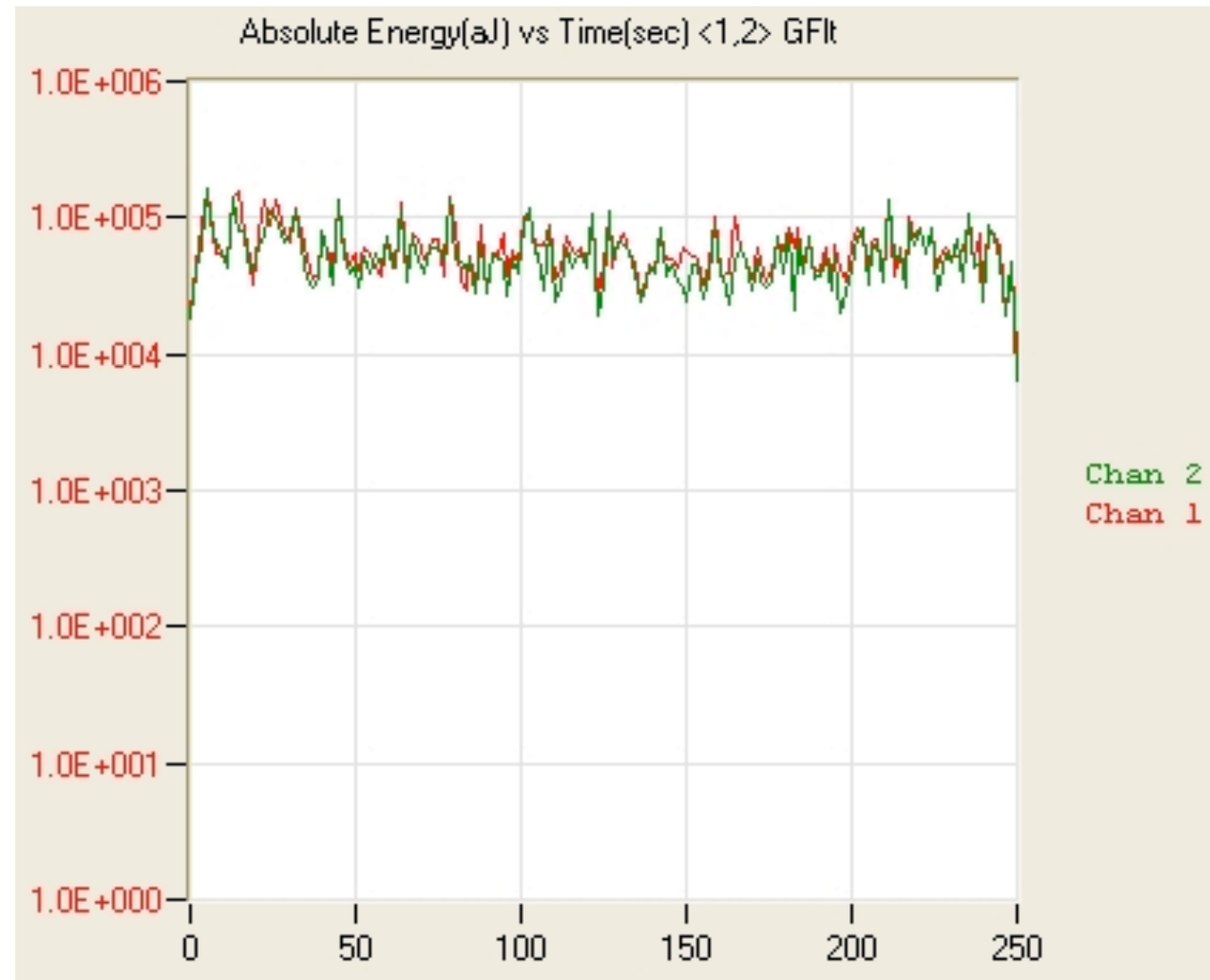

Figure 31: Absolute energy (aj) vs time (s) 


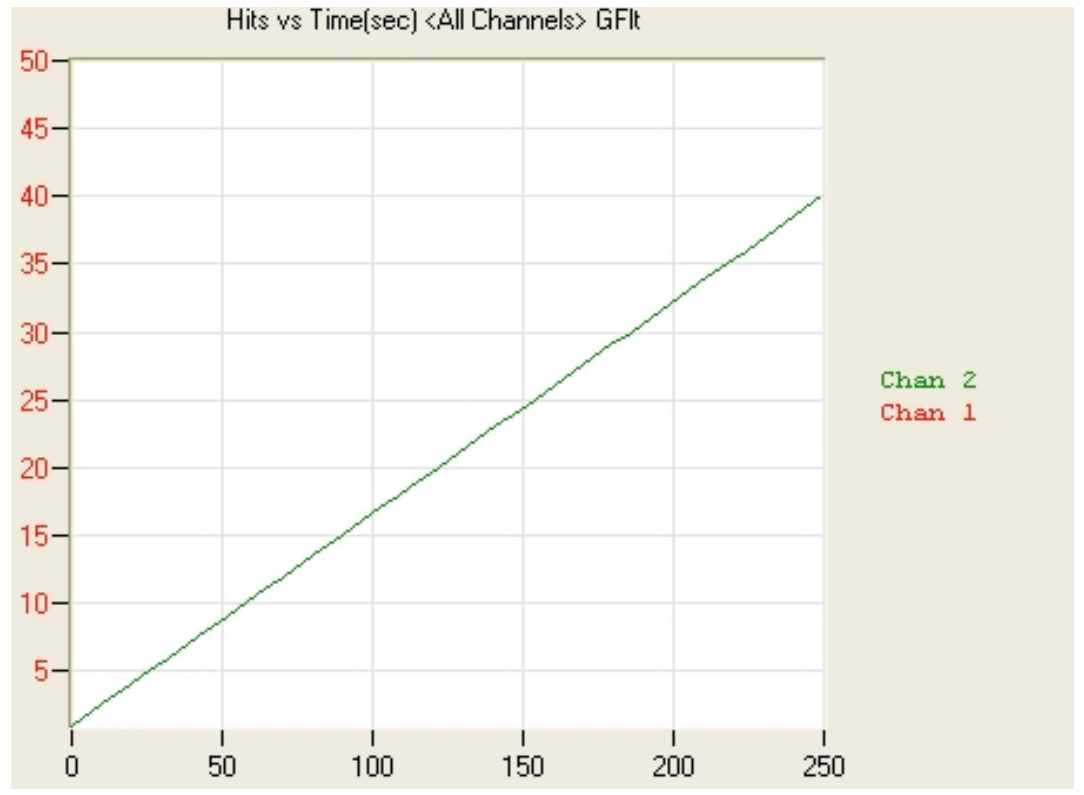

Figure 32: Hits vs time (s) in ICP test

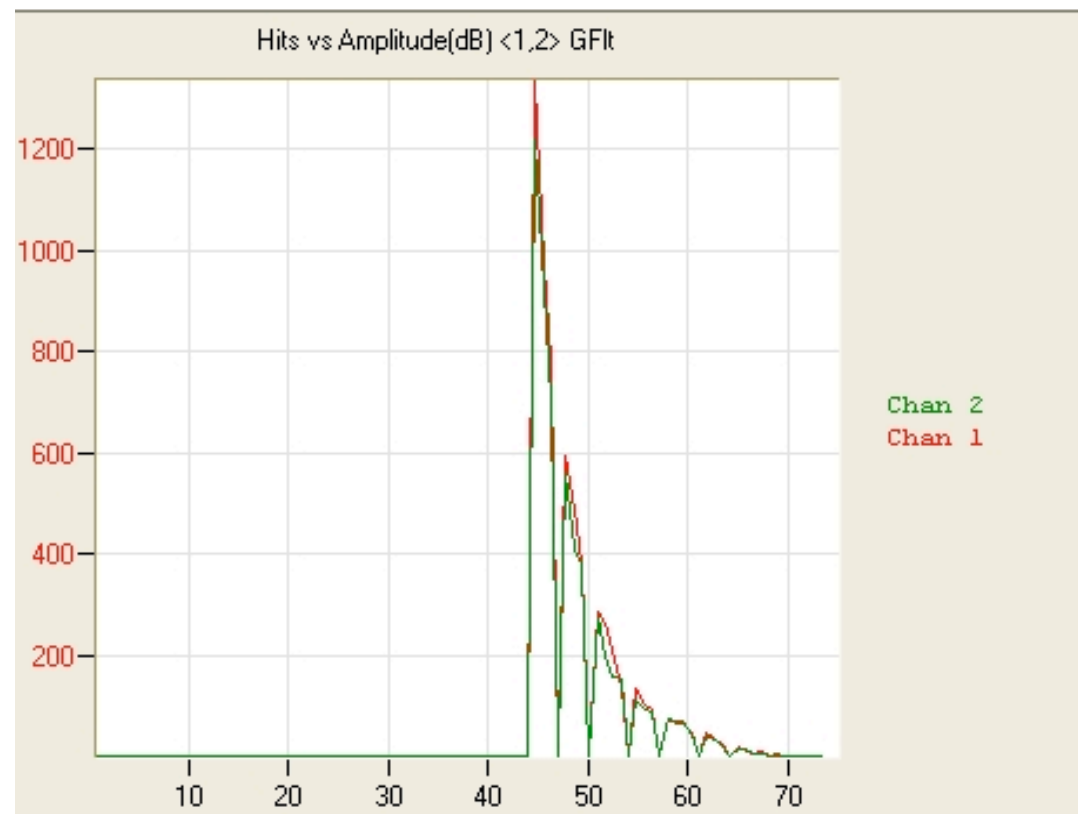

Figure 33: Hits vs amplitude (db) in ICP test 


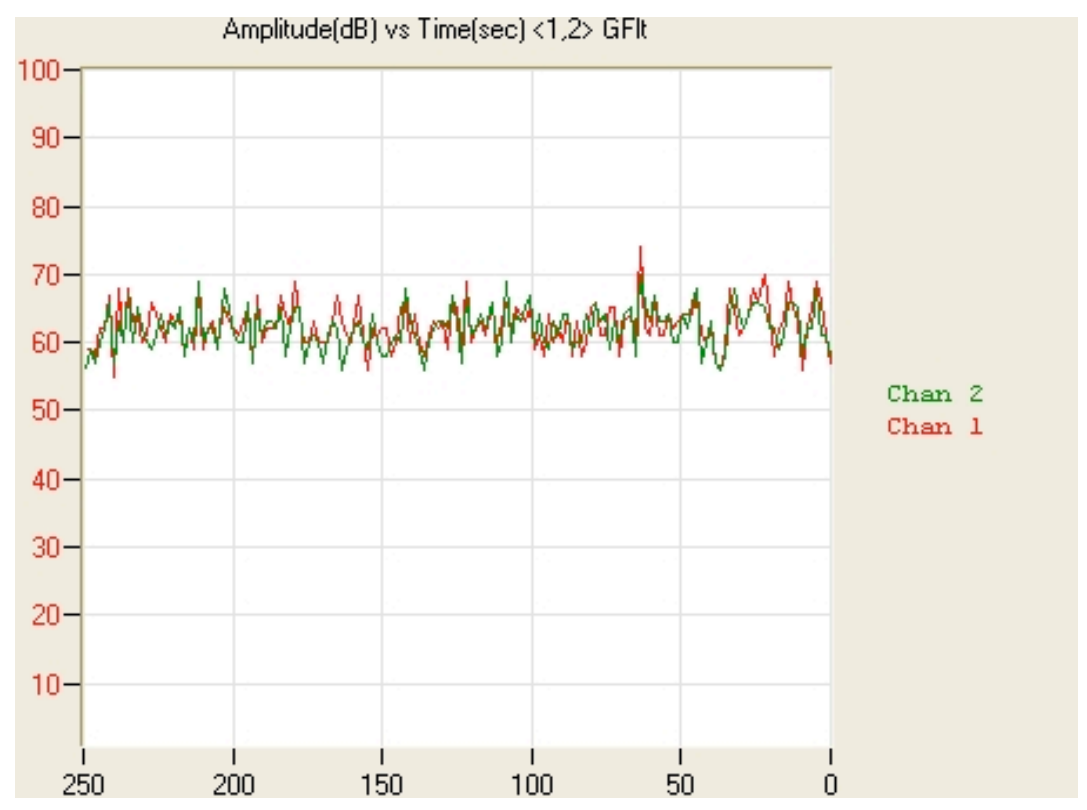

Figure 34: Amplitude vs time (s) in ICP test

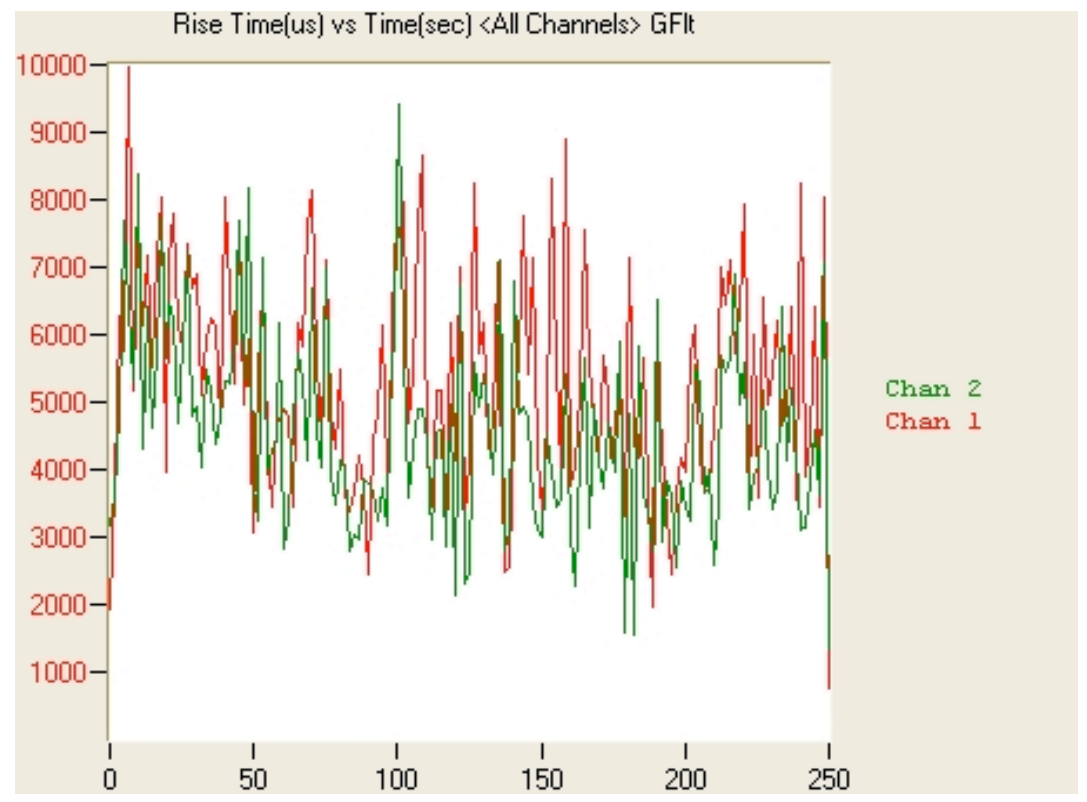

Figure 35: Rise time ( $\mu s)$ vs time (s) in ICP test 


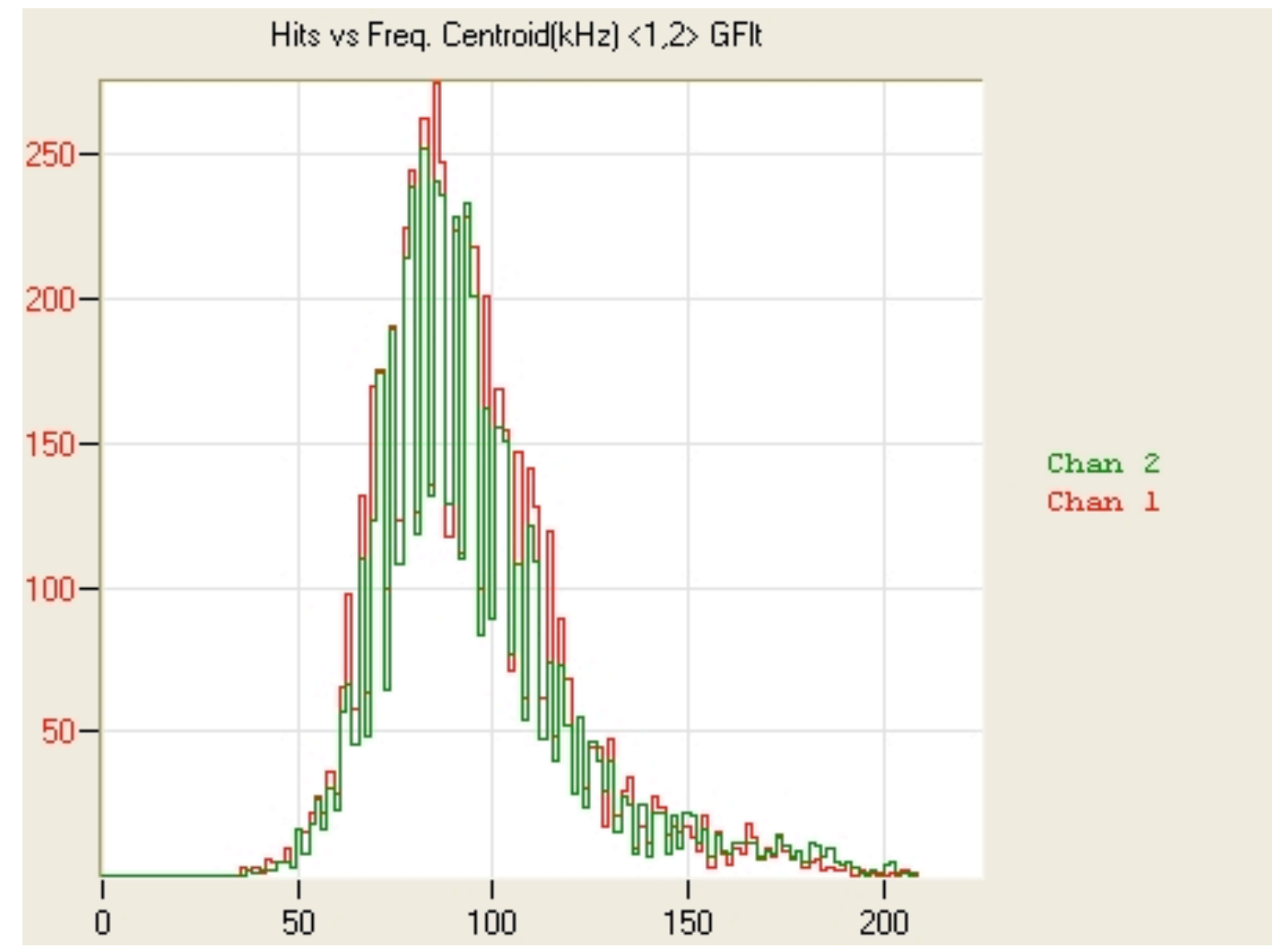

Figure 36: Hits vs frequency centroid $(\mathrm{kHz})$ in ICP test 


\subsubsection{Data Analysis}

The data from the seven trials (figure 37) are classified into two groups: argon based and nitrogen based plasma (figure 38).

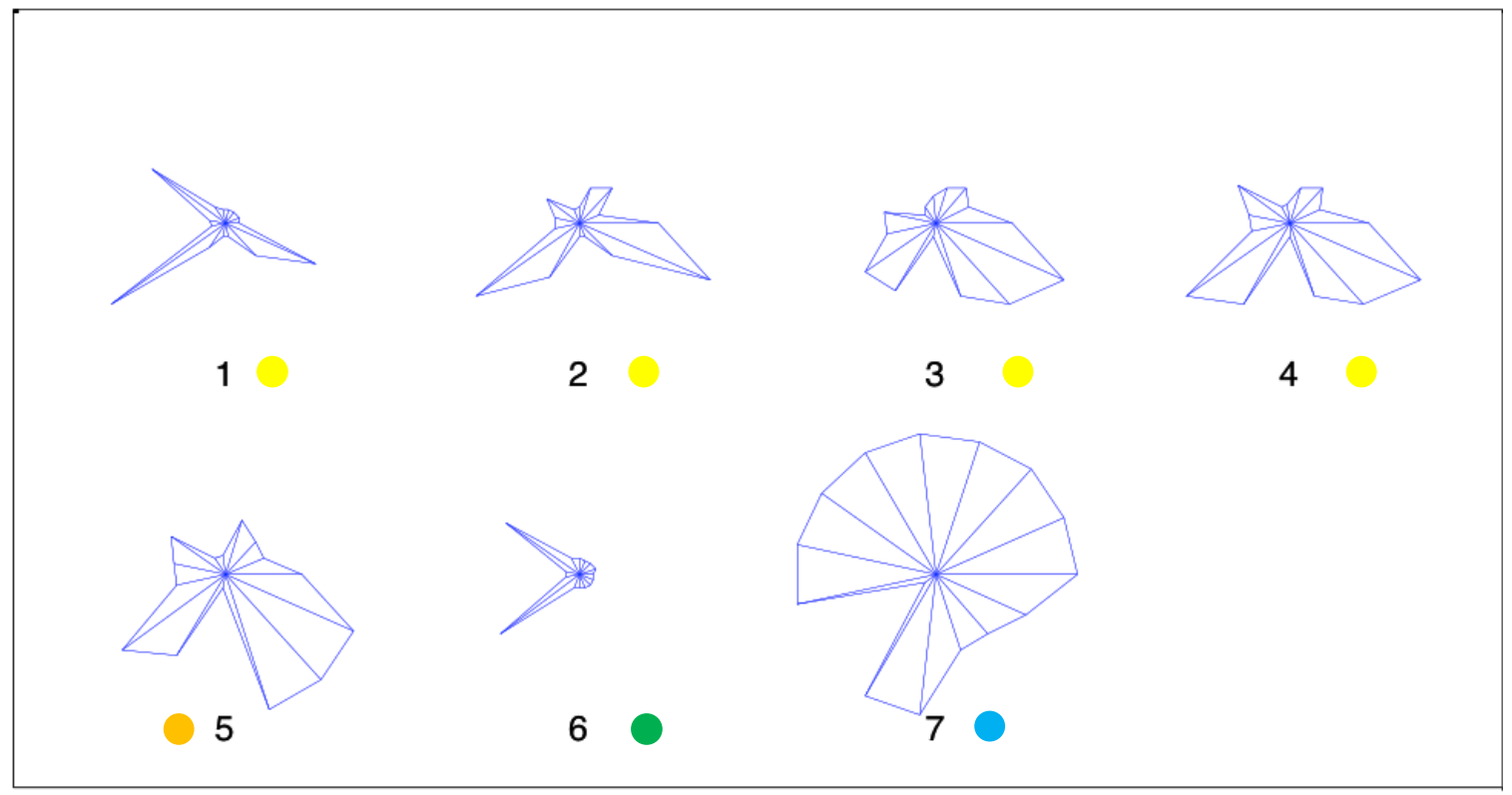

Figure 37: Labeled glyph plots of the seven trials in the plasma facility; yellow, 10 SLMP Ar / 30

SLMP N; orange, 40 SLMP N; green, 30 SLMP Ar / 10 SLMP N; cyan, 40 SLMP Ar

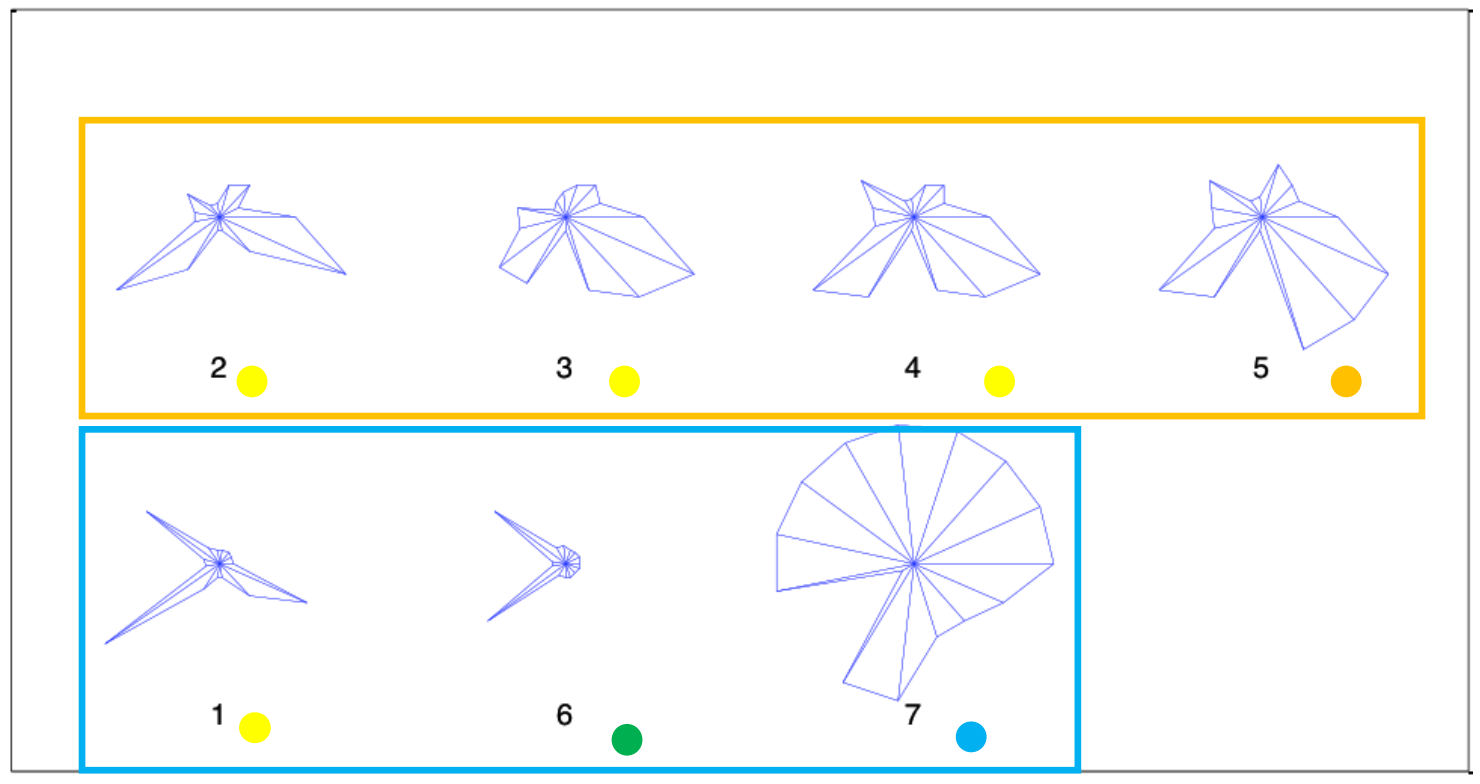

Figure 38: Classified trials with group labels; orange, $N$ based; blue, Ar based 


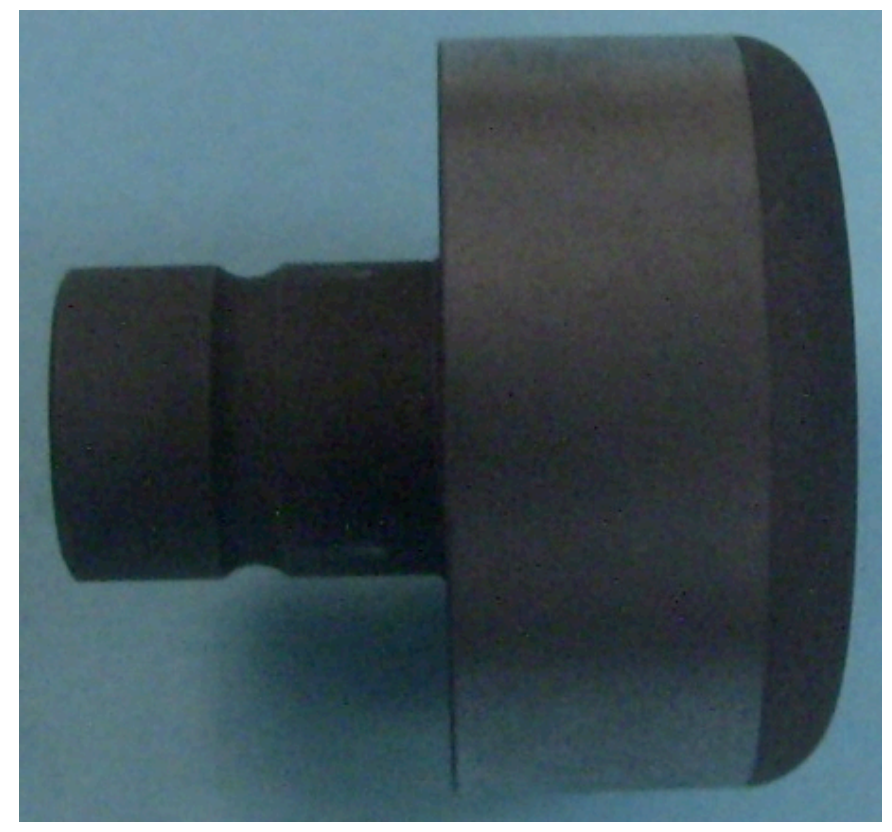

Figure 39: Graphite sample

The specific heat capacity or argon is $20.786 \mathrm{~J} \cdot \mathrm{mol}^{-1} \cdot \mathrm{K}^{-1}$; for nitrogen it is 29.124 $\mathrm{J} \cdot \mathrm{mol}^{-1} \cdot \mathrm{K}^{-1}$. The argon's liquid density is $1.40 \mathrm{~g} \cdot \mathrm{cm}^{-3}$, and the liquid nitrogen density of $0.808 \mathrm{~g} \cdot \mathrm{cm}^{-3}$

It appears that the pure argon-based trial is most energetic of the seven trials, with higher center frequencies and greater liquid density. Trial one (10 Ar SLMP / $30 \mathrm{~N}$ SLMP) is the only misclassified trial, with a predominately Nitrogen based plasma stream falling into the Argon based classification group. Trial one is also the least similar of the four 10 Ar SLMP / 30 N SLMP based trials with a similar initial frequency, and RMS as the other trials (features not part of classification), but lesser energy and higher center frequency (both classification features). The center frequency of trial one is higher 
than the four other nitrogen-based trials, and even higher than that of trial six with the higher argon composition. Trial one is also similar to trial six, with a higher average frequency and a similar energy. The argon based trials are dominated by a higher center frequency, with the purely argon trial dominating the energy category. The graphite sample loses mass each trial; trail one could be considered an outlier of this experiment possibly because the sample was still fresh. The graphite sample is similar to figure 39. Figure 40 is a photo of what is going on during the trials inside the plasma torch.

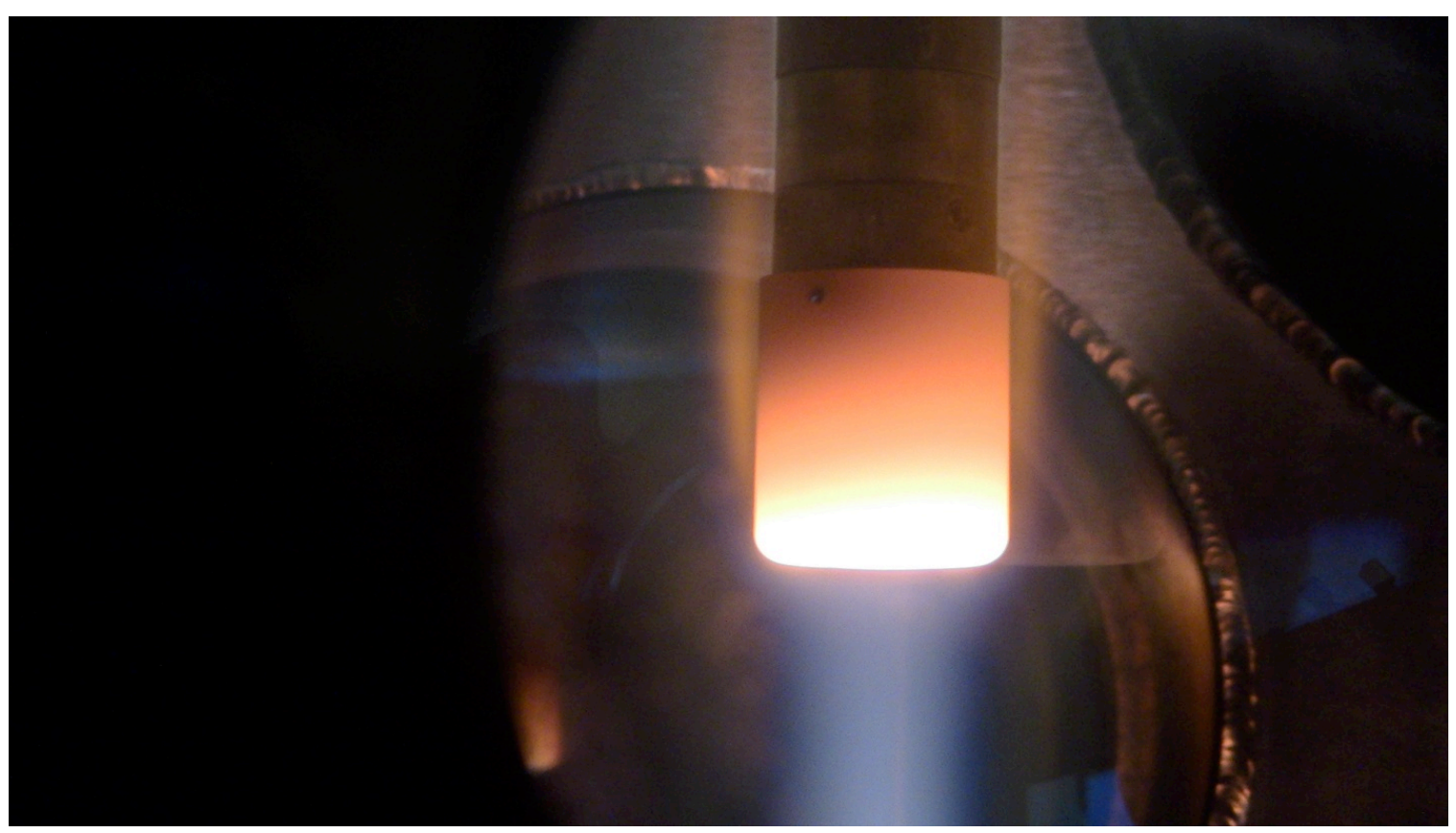

Figure 40: Inside the plasma torch, excited graphite sample

A MANOVA statistical analysis of the two classified groups is conducted in MATLAB [2]. The null hypothesis that the means of the two groups are the same multivariate vector of $n$ feature dimension is served by a p-value of 0.0043 . Only the five features used for classification (page 18) are analyzed with MANOVA. It is common to 
declare a result significant if its p-value is less than 0.01 . Thus, the null hypothesis can be rejected with a $95 \%$ confidence, leaving the possibility that the means could still be related linearly; the means thus lie in a 2-dimensional space [4].

The MANOVA analysis strengthens the validity of the classification; the low pvalue indicates independence between the means of the two groups, rejecting the null hypothesis of all the trials in one group, and supporting this reasonable classification of two groups. The two groups can be considered a useful representation of the data. A MANOVA analysis can also be used to test the validity of three groups of data (or more) [4]. 


\section{CHAPTER FIVE: WAVEGUIDE THEORY OF OPERATION}

\subsection{Linear Elastic Ball Model}

The motion of the steel ball waveguide can be modeled using a lumped-mass onedimensional chain system of linear springs and balls. For illustrative purposes figure 41 shows three balls are joined by springs and connected to a fixture as shown below.

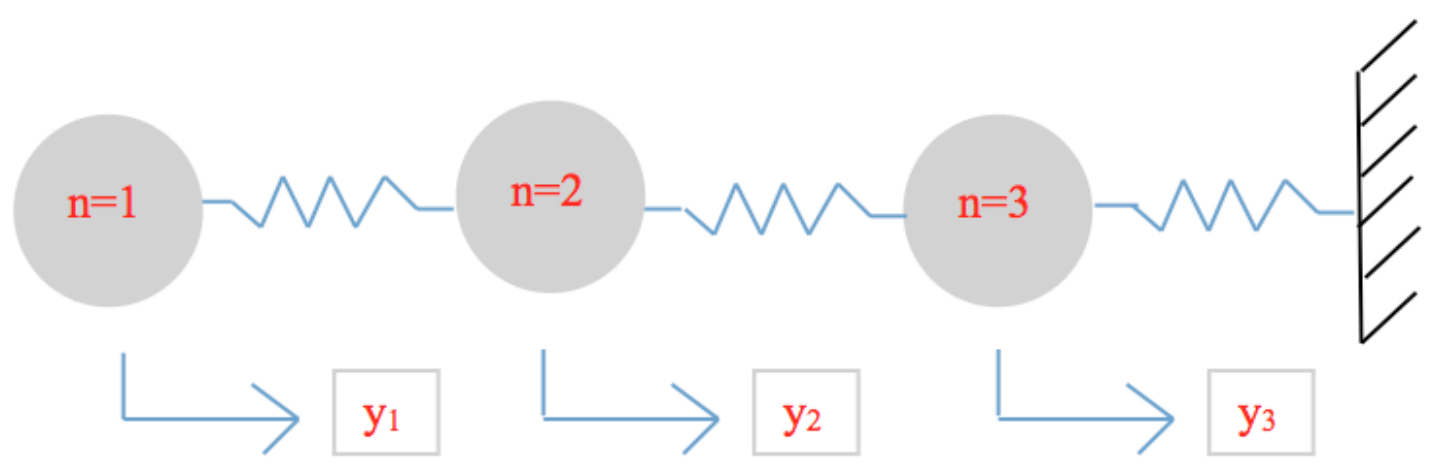

Figure 41: A one-dimensional three ball and spring linear system

Each ball has a mass $m$. Each spring has a spring constant $k$. For illustration purposes, in this particular model (figure 42, next page), the coefficient $\mathrm{k} / \mathrm{m}$ is equal to one. The velocity of the striker in the model is $0.7 \mathrm{in} / \mathrm{s}$, a velocity chosen so the motion of the balls doesn't max out the springs. 


\section{Position (in)}

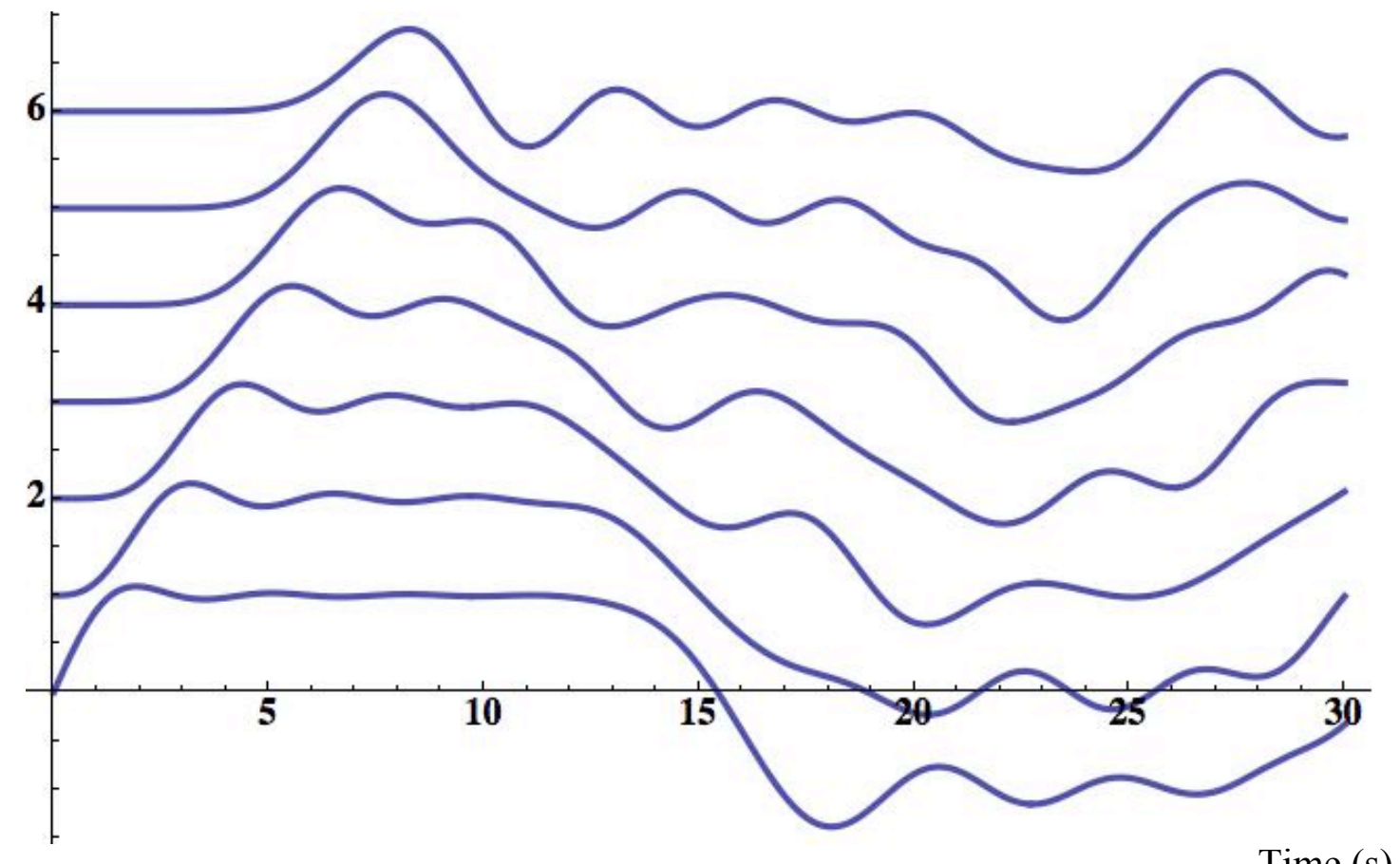

Time (s)

Figure 42: The positions of seven balls in an excited linear spring-ball chain model vs. time

The wave speed in this model is calculated to be approximately 0.78 inches per second, closely matching the velocity of the striker. The wave speed is calculated as the slope of the line connecting the maxima of balls one and six. The purpose of these simulations is to closely model the motion of a steel ball dimer chain using a linear system of springs and balls. The above model, while simple due to its linear nature can be considered a good prediction of the motion experienced by a steel ball dimer chain. Different choices of the $\mathrm{k} / \mathrm{m}$ ratio will produce different wave speeds. 


\subsection{Hertz Law for Nonlinear Interaction}

The model and results presented in section 5.1 assume linear elastic interactions between the balls. However, it is well known that elastic bodies, such as spheres, in contact exhibit nonlinear interactions. The Hertz theory of contact elasticity is an accepted model for these nonlinear interactions. The theory for nonlinear solitary waves traveling through a dimer chain is based upon Hertz's law, equations 7 and 8. This law defines the interaction between two particles as [5]:

$$
\begin{gathered}
F=A \delta^{3 / 2} \\
A=\frac{E \sqrt{a}}{3\left(1-v^{2}\right)}
\end{gathered}
$$

\section{Equations 7 and 8: Hertz Law}

$F$ is the compression force experienced between the particles, $\delta$ is the least difference between particle centers, $A$ is a coefficient, $a$ is the diameter of the beads in the chain, $v$ is the Poisson's ratio of the beads, and $E$ is the Young's Modulus of their material. In this model the Young's modulus of the steel particles is approximated as 200 GPA and the Poisson's ratio is approximated as 0.3 . 
The nonlinear contact interaction experienced between the beads is the catalyst for the formation of propagating solitary waves. It has been found in a certain experiment that when the wavelength of the propagating wave is much larger than the particle diameters the speed of the waves depends on the dynamic strain, defined by the dynamic force between the particles [5].

\subsubsection{Modified Model for Hertzian Interaction}

The theoretical acceleration experienced at a $\mathrm{j}^{\text {th }}$ particle center can be calculated with the modified model for Hertzian interaction, equations $9-13$ on this page and the next.

$$
\begin{gathered}
\ddot{y}(t)_{j}=\frac{A_{j-1, j}}{m_{j}} \delta_{j}^{3 / 2}-\frac{A_{j, j+1}}{m_{j}} \delta_{j+1}^{3 / 2}+g \\
A_{j, j+1}=\frac{4 E_{j} E_{j+1}\left(\frac{R_{j} R_{j+1}}{R_{j}+R_{j+1}}\right)^{1 / 2}}{3\left[E_{j+1}\left(1-v_{j}^{2}\right) E_{j}\left(1-v_{j+1}^{2}\right)\right]}
\end{gathered}
$$

\section{Equations 9 and 10: Modified model for Hertzian interaction [6]}

$R_{j}$ is the radius of the $\mathrm{j}^{\text {th }}$ particle; $v_{j}$ is the Poisson's ratio of the $\mathrm{j}^{\text {th }}$ particle; $E_{j}$ is the Young's Modulus of the $\mathrm{j}^{\text {th }}$ particle. If the configuration of the chain is verticle, the acceleration of gravity $g$ is $9.81 \mathrm{~m} / \mathrm{s}^{2}$. The mass $m_{j}$ of each of the steel balls is $0.0163 \mathrm{~kg}$. 
The particle material in the waveguide consists of all steel balls with Young's Moduli of 200 GPA, Poisson's ratio of 0.30 , and radii of $16 \mathrm{~mm}$ :

$$
\begin{gathered}
\delta_{1}=0 \\
\delta_{j}=\max \left\{y_{j-1}-y_{j}, 0\right\} \\
\delta_{n+1}=\max \left\{y_{j}, 0\right\}
\end{gathered}
$$

Equations 11, 12, and 13: Modified model for Hertzian interaction [6]

The equilibrium positions of the three particles in a vertically oriented dimer chain are calculated with equation 14 . Boundary conditions arise at the ends of the chain. The displacement of the wall $\left(u_{k+1}{ }^{\text {st }}\right.$ particle $)$ is zero. The initial positions of the three particles are found to be $6.33 \mathrm{~m}, 4.96 \mathrm{~m}$, and $2.82 \mathrm{~m}$ respectively:

$$
u_{k}=\left(\frac{\sum_{j=1}^{k} m_{j} g}{A_{k, k+1}}\right)^{2 / 3}+u_{k+1}
$$

Equation 14: Static equilibrium displacements of some $\mathrm{k}^{\text {th }}$ particle in a dimer chain model [6] 
The solution to this system of equations is calculated with Mathematica software (figure 43). The three particles are modeled with initial velocities $-1 \mathrm{~m} / \mathrm{s}$, zero and zero respectively.

\section{Position (m)}

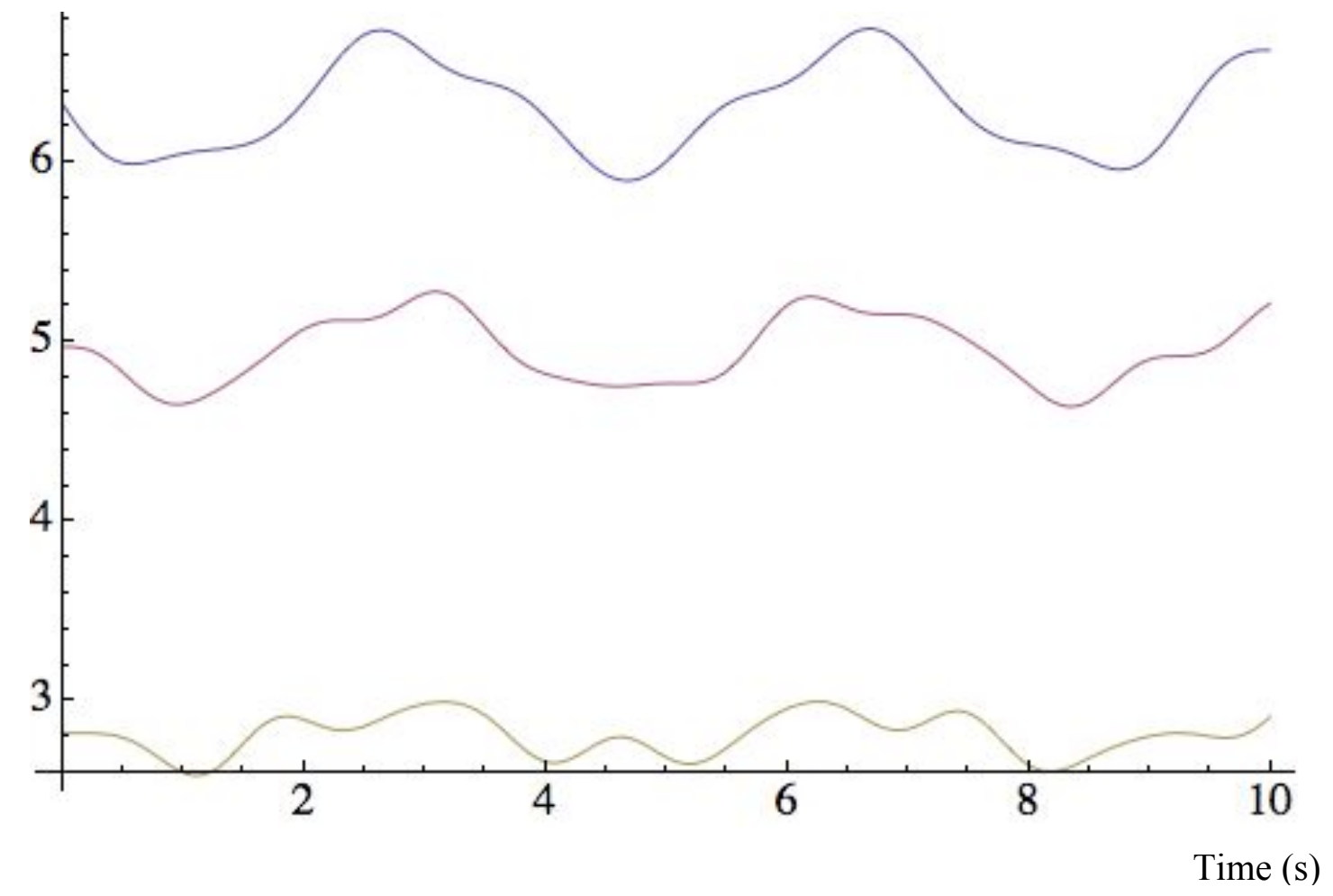

Figure 43: The positions of balls one (blue), two (red), and three (green) in the nonlinear model vs. time

The wave speed in the above simulation is approximately $48 \mathrm{~mm} / \mathrm{s}$. 


\subsection{Wave Velocity Approximation}

The velocity of the wave traveling through the dimer chain can be approximated with equation $15[5]$ :

$$
V=0.6802 *\left(\frac{2 E}{a \rho^{\frac{3}{2}}\left(1-v^{2}\right)}\right)^{1 / 3} F_{m}^{1 / 6}
$$

\section{Equations 15: Wave Velocity in a Dimer Chain}

$E$ is the Young's modulus of the particles, $a$ is the diameter of the particles, $\rho$ is the density of the material, $v$ is poison's ratio of the material, and $F_{m}$ is the maximum applied contact force between the particles. For a steel ball waveguide experiencing a peak force of $0.452 \mathrm{mN}$ the wave speed is $63.5 \mathrm{~m} / \mathrm{s}$. This force is an estimation of the peak force applied with a 1 volt striker amplitude at $174 \mathrm{kHz}$.

The shapes of these waves can be modeled by equations 16 and 17 [3]: 


$$
\xi=\left(\frac{5 V^{2}}{4 c^{2}}\right) \cos ^{4}\left(\frac{\sqrt{10}}{5 a} x\right)|x| \leq 0.0394
$$

$$
c=\sqrt{\frac{2 E}{\pi \rho\left(1-v^{2}\right)}}
$$

\section{Equations 16 and 17: Wave Shape in a Dimer Chain}

$x$ is the axis of wave propagation; $\xi$ represents the shape of the wave within five particle diameters from the origin. The shape depicted in figure 44 spans almost exactly 5 balls of $5 / 8$ " diameter. It takes five or more balls to completely experience this motion.

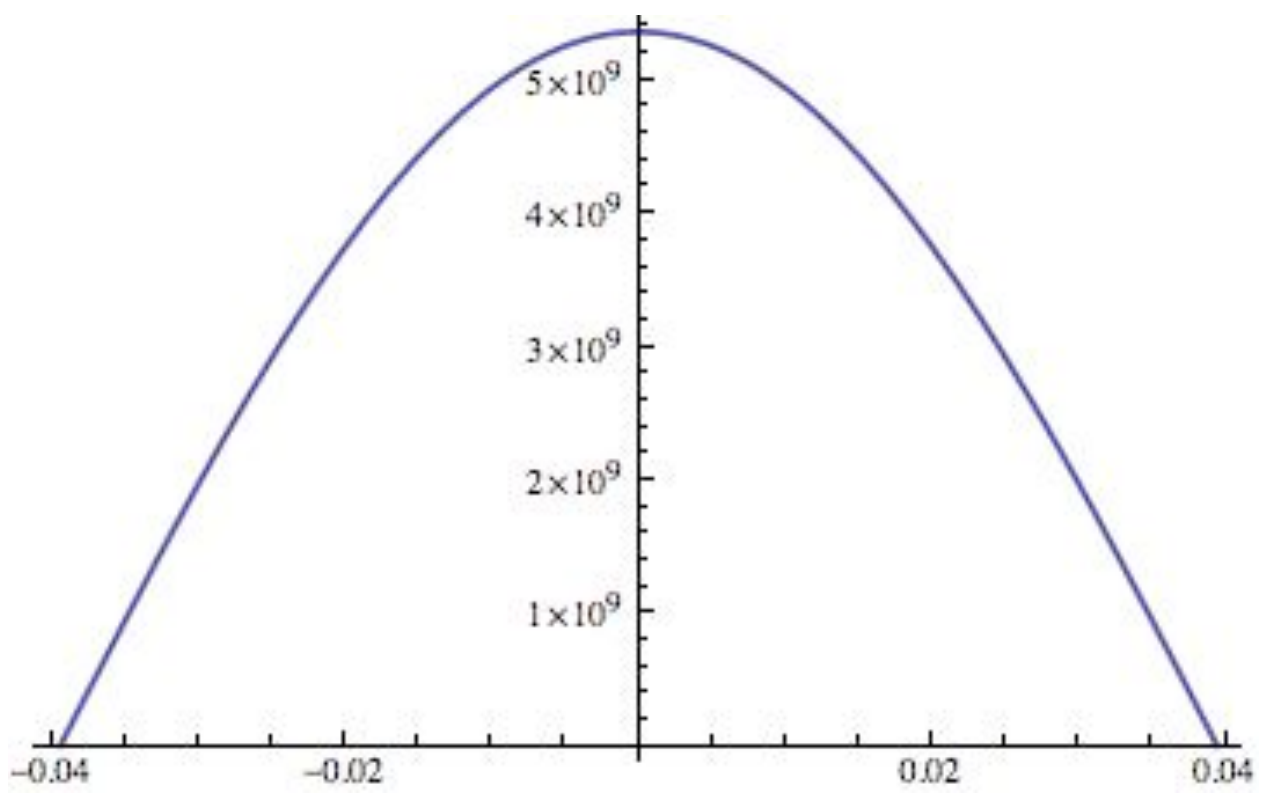

Figure 44: Shape of the wave inside a chain vs. axis of wave propagation 


\subsection{Reciprocal Approximation}

The frequency of the highly nonlinear solitary wave in the waveguide can be approximated as the reciprocal of the time duration for the wave to travel from transducer to transducer (equation 18). This representation is for linear waves. A transducer (PAC $\mathrm{R} 15 \alpha$ ) vibrating at $10 \mathrm{kHz}$ with amplitude of $1 \mathrm{~V}$ is coupled to a seven-ball waveguide positioned vertically. The duration of travel from one transducer to the other is calculated as $398 \mathrm{~ms}$.

$$
f=\frac{v}{2 d}=\frac{1}{2 t}
$$

Equation 18: Reciprocal Approximation

The resulting frequency based upon the above equation is $1.26 \mathrm{~Hz}$. This difference between the striker frequency and the above frequency is probably due to the non-linear properties of the waveguide. 


\section{CHAPTER SIX: NONLINEAR WAVEGUIDE DISCRIMINATION}

\subsection{Test Setup and Procedure}

This chapter describes the results of a series of tests that were designed to measure and discriminate the behavior of ball-chain waveguides of various constructions. Copper waveguides filled with steel balls are used to study the wave propagation of a linear sine wave chirp waveform. A straight copper pipe is filled with 33 steel balls and secured horizontally with respect to the ground. Two transducers at either end of the waveguide hold the balls in place with a negligible static force along the one-dimensional axis of the chain. A striker transducer is used to excite the balls with the linear chirp waveform while a second transducer is used to collect acoustic emissions data at the opposite end of the chain. The waves propagating through the chain of balls are known as highly nonlinear solitary waves (HNSW) [3].

Balls of five different materials are incorporated into this experiment: steel, nitrile rubber, neoprene, cork, and marble; the balls are all of 5/8" in diameter matching the diameter of the copper tube waveguide. The effects of the two types of rubber, cork, and marble balls with respect to the propagating waveform through the particle chain are investigated by inserting one of each type in between steel balls, one at a time, at the center of the waveguide. Material properties for these balls can be found in table 5 on the following page. 


\begin{tabular}{|l|l|l|}
\hline Ball Type & Young's Modulus (Gpa) & Damping Ratio \\
\hline Steel & $200-216$ & $0.001-0.002$ \\
\hline Nitrile & $0.0015-0.0025$ & $\sim 0.05$ \\
\hline Cork & $0.013-0.05$ & $0.05-0.06$ \\
\hline Neoprene & $0.0007-0.002$ & $\sim 0.05$ \\
\hline Marble & $64-110$ & \\
\hline
\end{tabular}

Table 5: Young's modulus and damping ratio for the five materials [7] [8]

The linear chirp waveform sweeps from 50 to $550 \mathrm{~Hz}$ every half a second with an amplitude of $250 \mathrm{mV}$. This signal is linearly amplified by a gain of $+19.99 \mathrm{~dB}$ through a scaling amplifier (Stanford Research Systems (SRS) SIM983 scaling amplifier) and fed to the transducer (PAC R15 $\alpha$ ) acting as an actuator. The signal is also split, amplified by a gain of $+0.20 \mathrm{~dB}$ through a second scaling amplifier and fed to the PAC PCI-2 board for data acquisition. The chirp waveform is collected by a preamplifier (20/40/60 PAC preamplifier) and triggers a second transducer to report acoustic emissions data to the acquisition unit. The frictional forces of the transducer setup hold the chain of balls together (figure 45). 


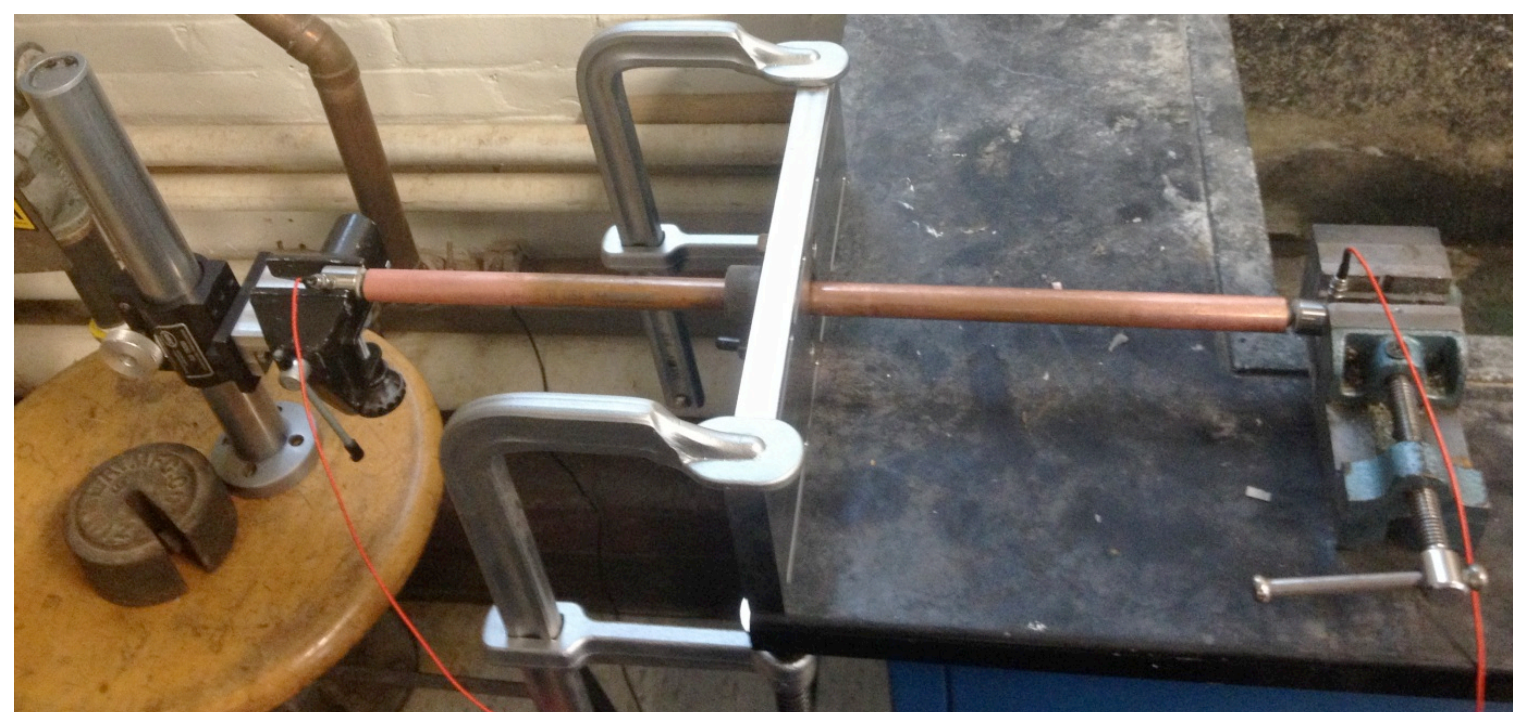

Figure 45: The experimental setup with actuating transducer, left; and sensing transducer, right

25 trials are acquired with the acoustic emissions acquisition unit. Of the 25 trials, ten are incorporated in a training matrix used to classify the other 15 trials to investigate the difference in the acoustic properties of the balls. The PAC data acquisition unit with the AEWin software collects fifteen acoustic classification features detailed in table one. 


\subsection{Chapter Results}

The results for the all steel ball trial display a portion of the sine chirp waveform and the sensing transducer's response with corresponding power vs. frequency plots (figure 46 ). The voltage of the sine wave chirp is $\sim 5$ volts. The voltage amplitude of the sensing transducer is approximately $0.4 \%$ of the voltage amplitude of the sine wave chirp at $\sim 20 \mathrm{mV}$. This is possibly due to energy dissipation and frictional losses or weak contact between the balls. The dominant frequency of the response of the sensing is greater than that of the chirp waveform; the power at its peak power frequency is approximately 10000 times less at a difference of $\sim 55 \mathrm{~dB}$.
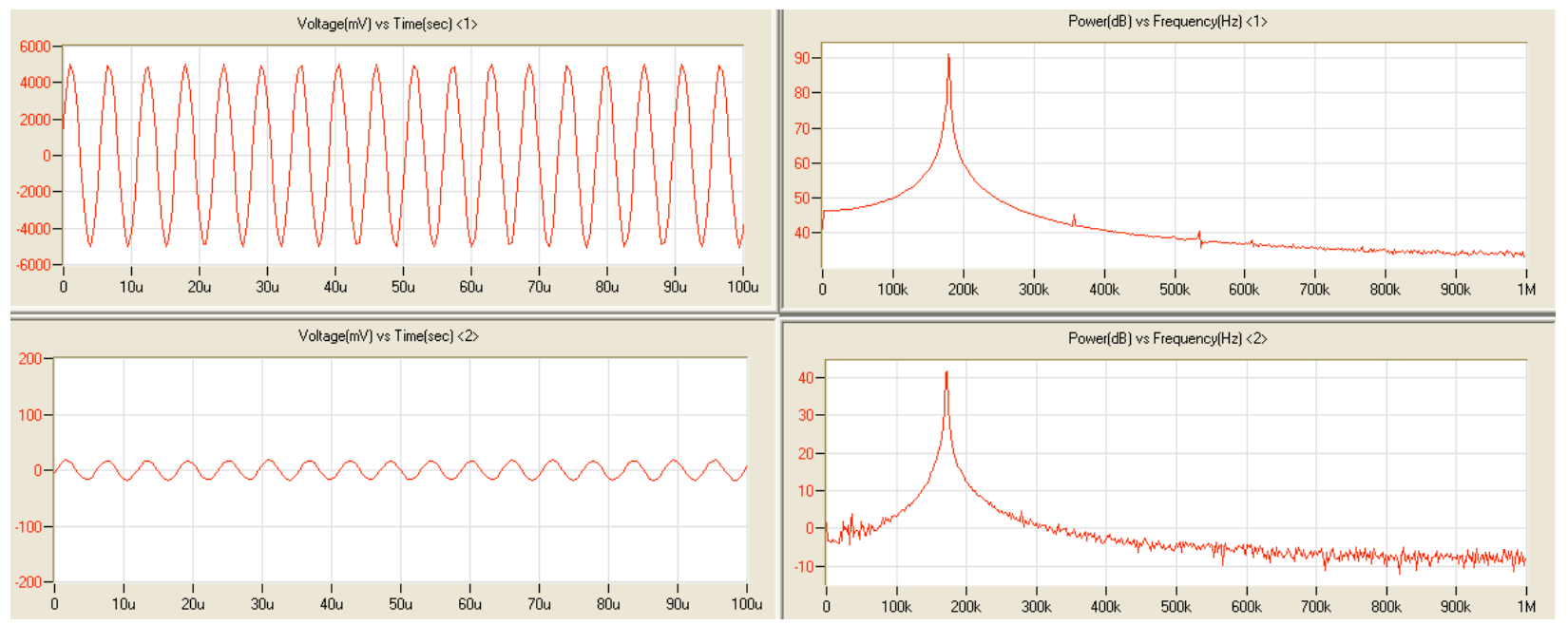

Figure 46: Voltage vs. time graphs for a portion of the chirp waveform $<1>$ and the sensing transducer $<2>$ with corresponding power vs. frequency graphs for the all steel ball trial 
Waveform streaming of the chirp waveform (figures 47-51, pages 58-60) alongside the output of this sensing transducers output reveals similar results across the trials with different peak amplitudes and different damping characteristics per ball type.

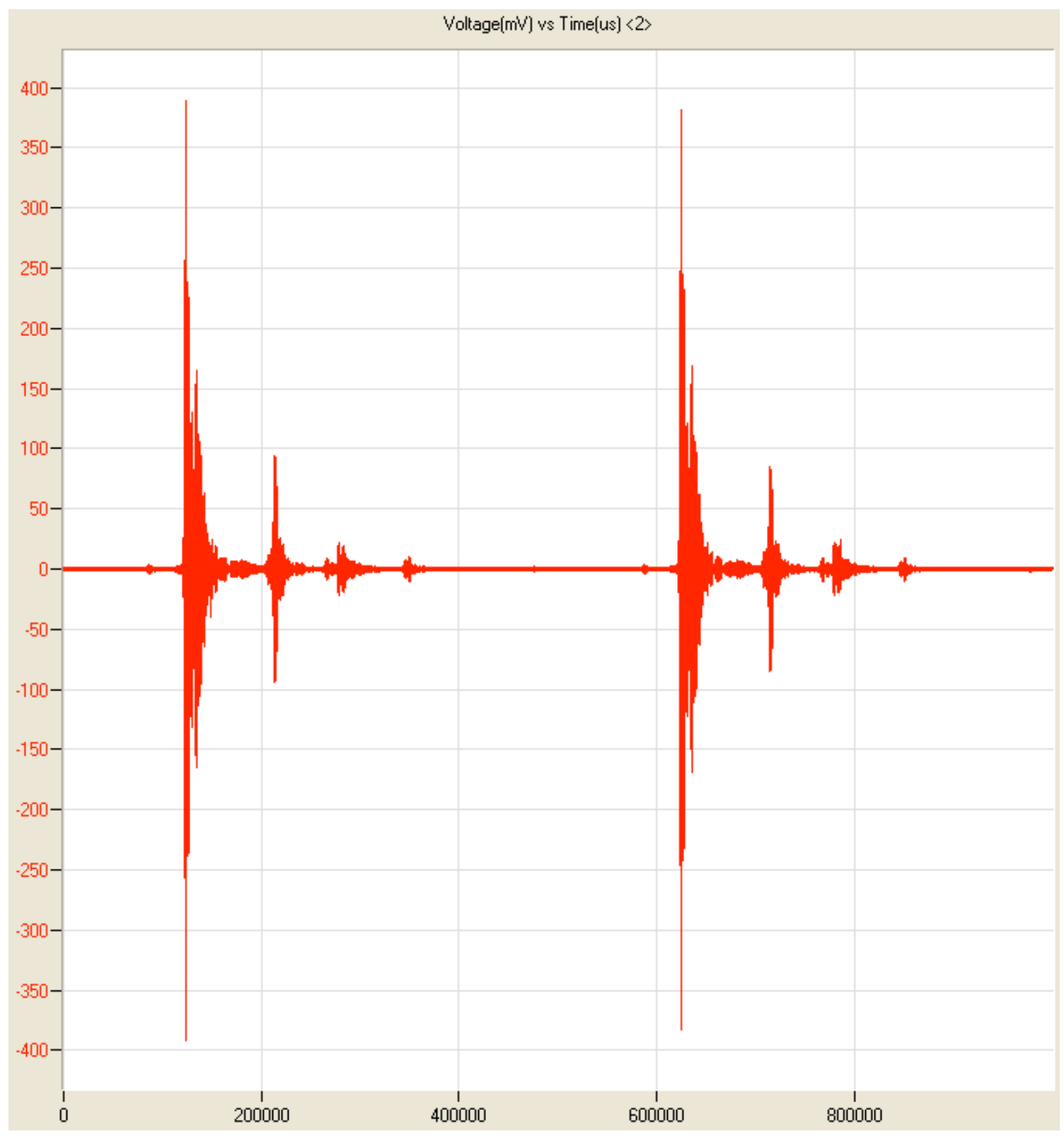

Figure 47: Resulting output voltage of the receiving transducer across a one second period for the all steel ball trial 


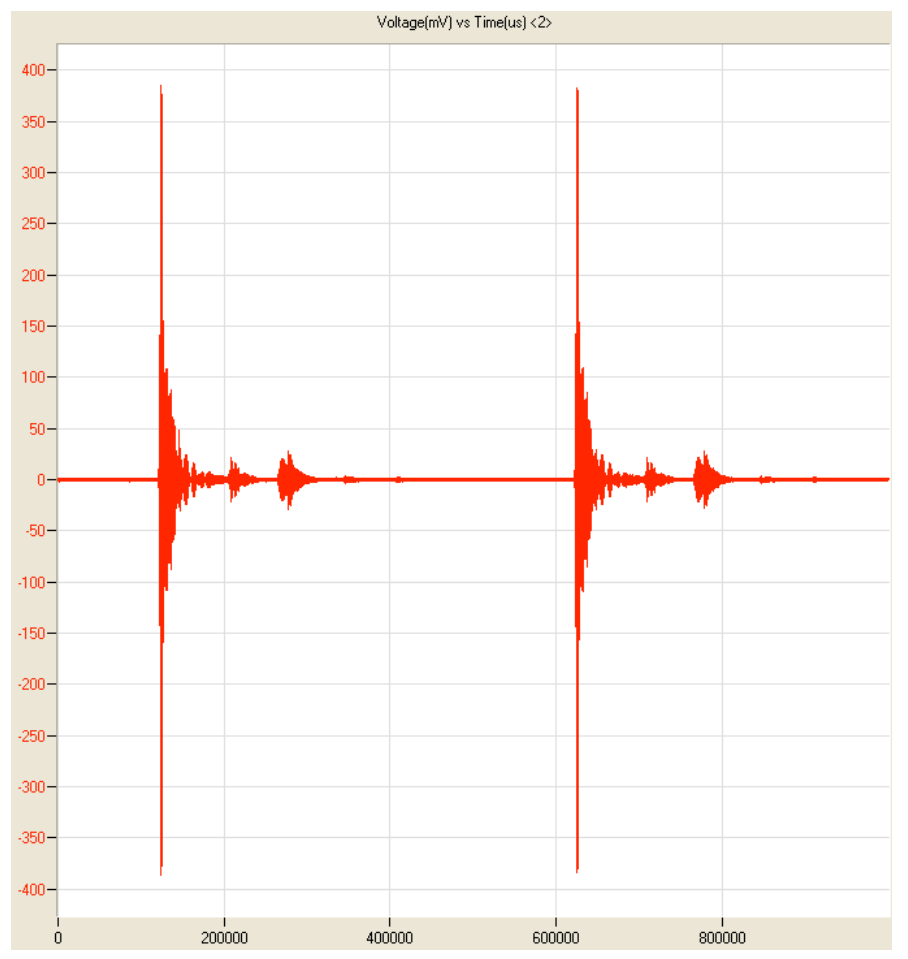

Figure 48: Resulting output voltage across a one second period for the rubber ball trial

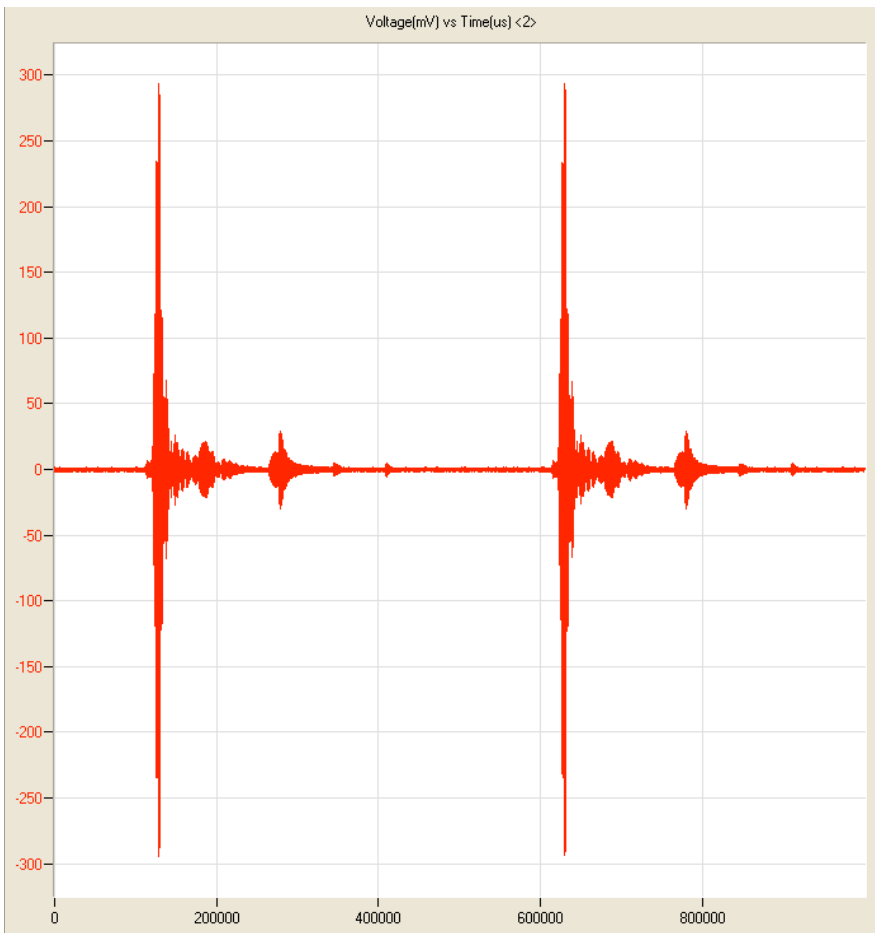

Figure 49: Resulting output voltage across a one second period for the cork ball trial 


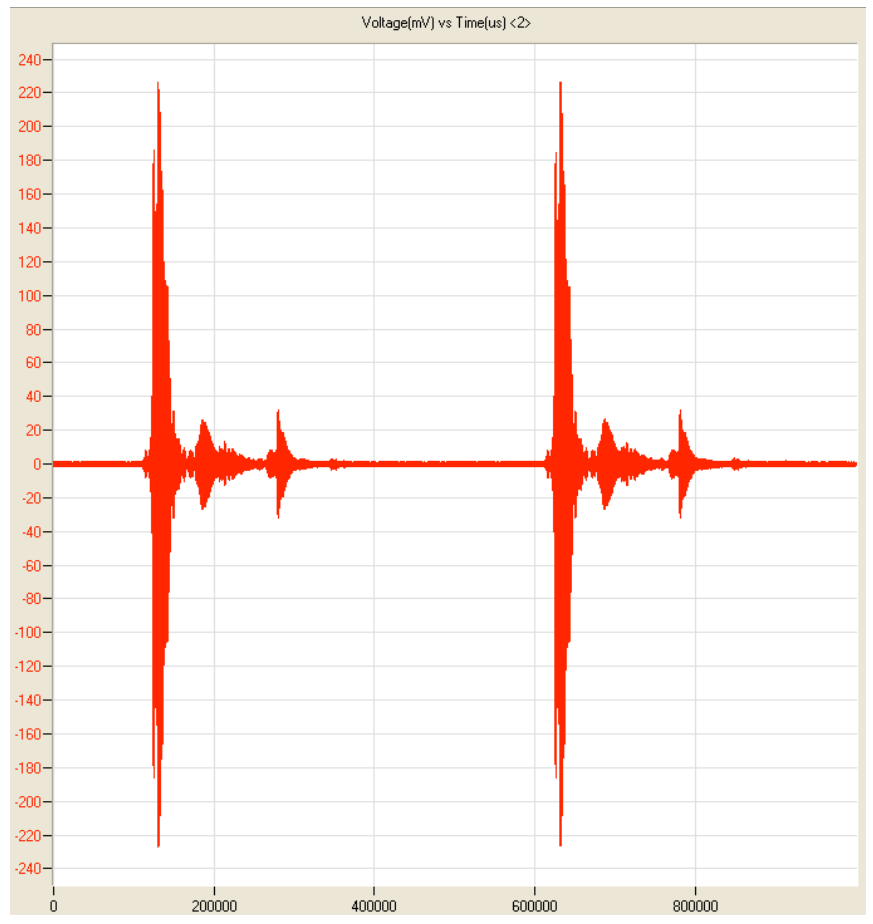

Figure 50: Resulting output voltage across a one second period for the neoprene ball trial

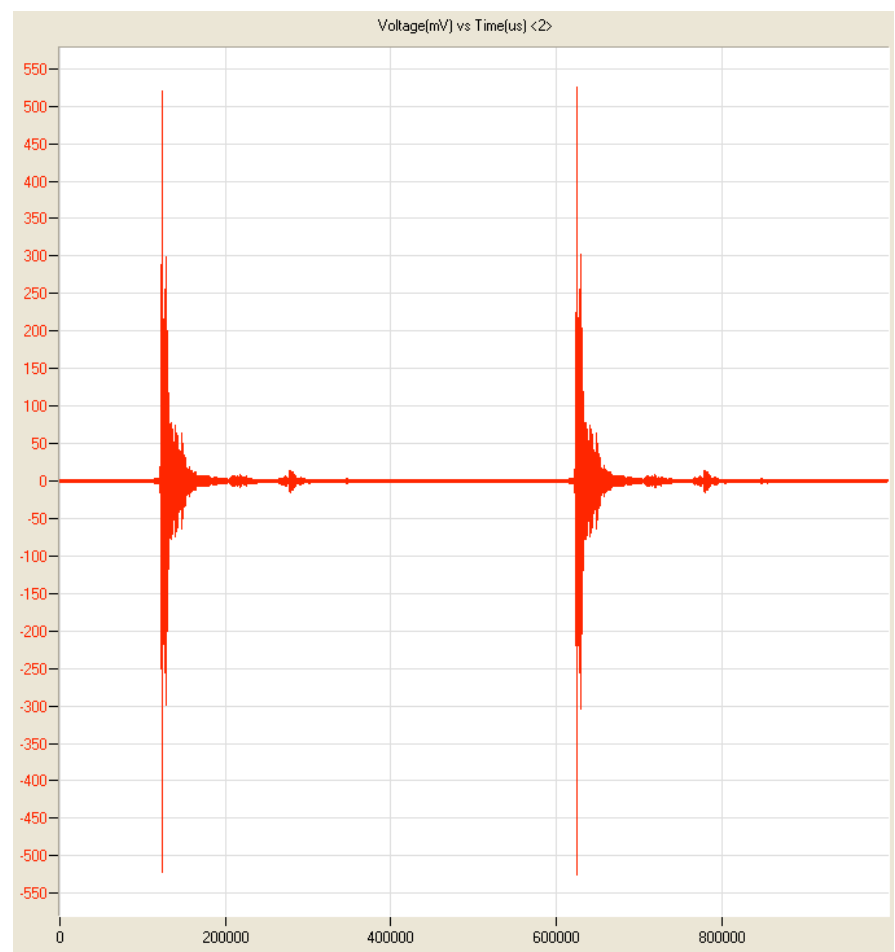

Figure 51: Resulting output voltage across a one second period for the marble ball trial 
These results can be compared to a similar trial of an L-shaped waveguide with a graphite sample head and no particle chain (figure 52). The results appear to be less grouped but of similar shapes and magnitudes with a stronger second group as opposed to the small second group seen in the previous figures. The second group is probably due to reflected waves between the transducers.

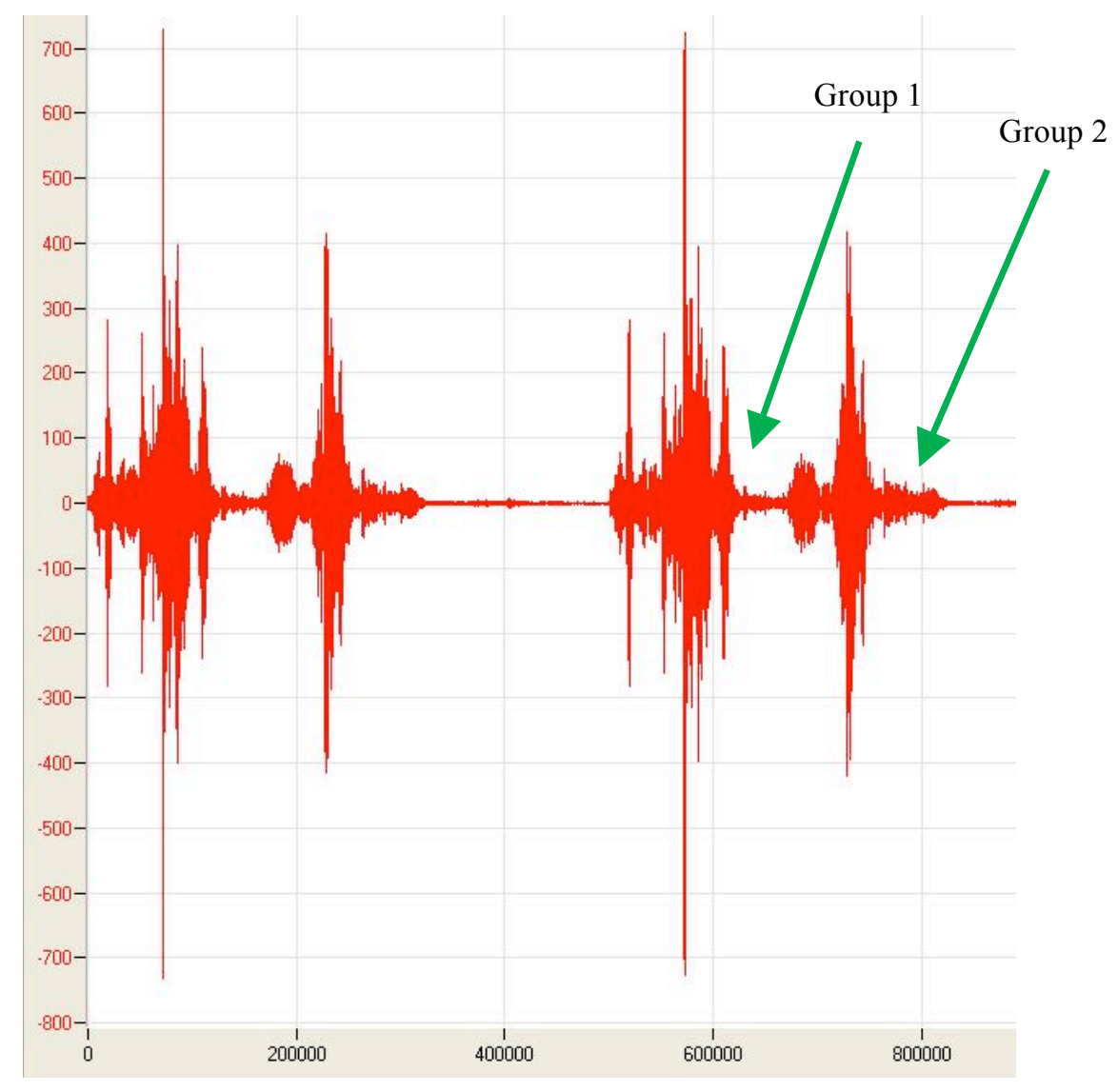

Figure 52: Resulting output voltage across a one second period for an L-shaped waveguide with a graphite sample head without a ball chain 


\subsection{Trial Discrimination and Analysis}

The trials are discriminated by the same five key elements: energy, rise time, absolute energy, center frequency, and signal strength. The results are arranged in glyph plots featuring the 15 acoustic characteristics in table one and grouped by class [2]. The five key elements are spokes three, five, eight, seven, and nine on the glyph plot in figure 12, page 19 .

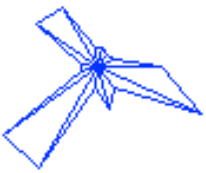

1

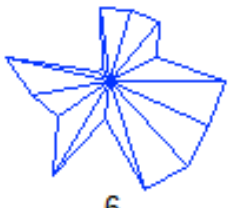

6

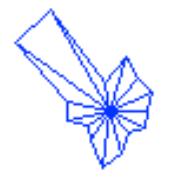

11

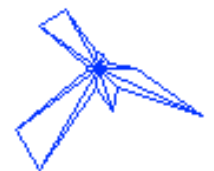

2
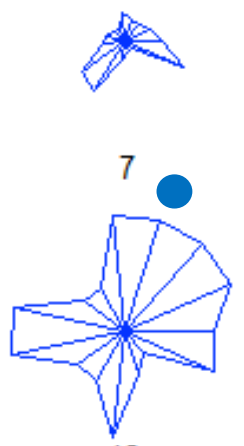

12

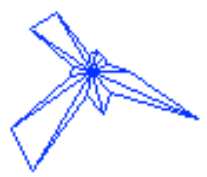

3

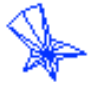

8

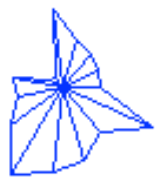

13
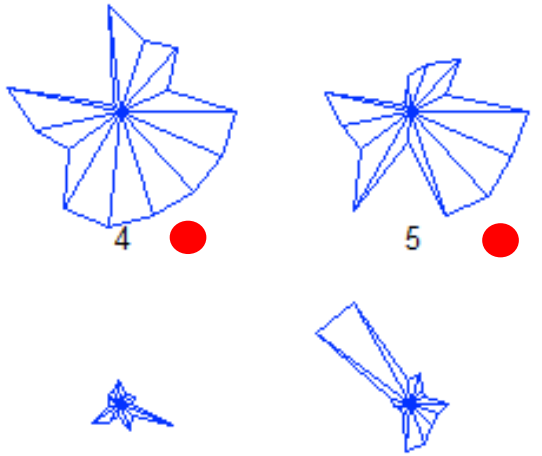

9

10

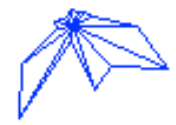

14

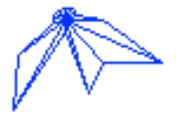

15

\begin{tabular}{|cc|}
\hline steel & neoprene \\
nitrile & marble \\
cork & Key \\
\hline
\end{tabular}

Figure 53: Glyph plots of the 15 trials for discrimination with their actual trial type 
The above glyph plots in figure 53 are from the 15 trials for discrimination outlined with their actual types. The glyphs in figure 54 (next page) are the same as above but classified into groups; the discriminated groups are highlighted and the group type is distinguished by the same color code as used to distinguish the actual trial types.
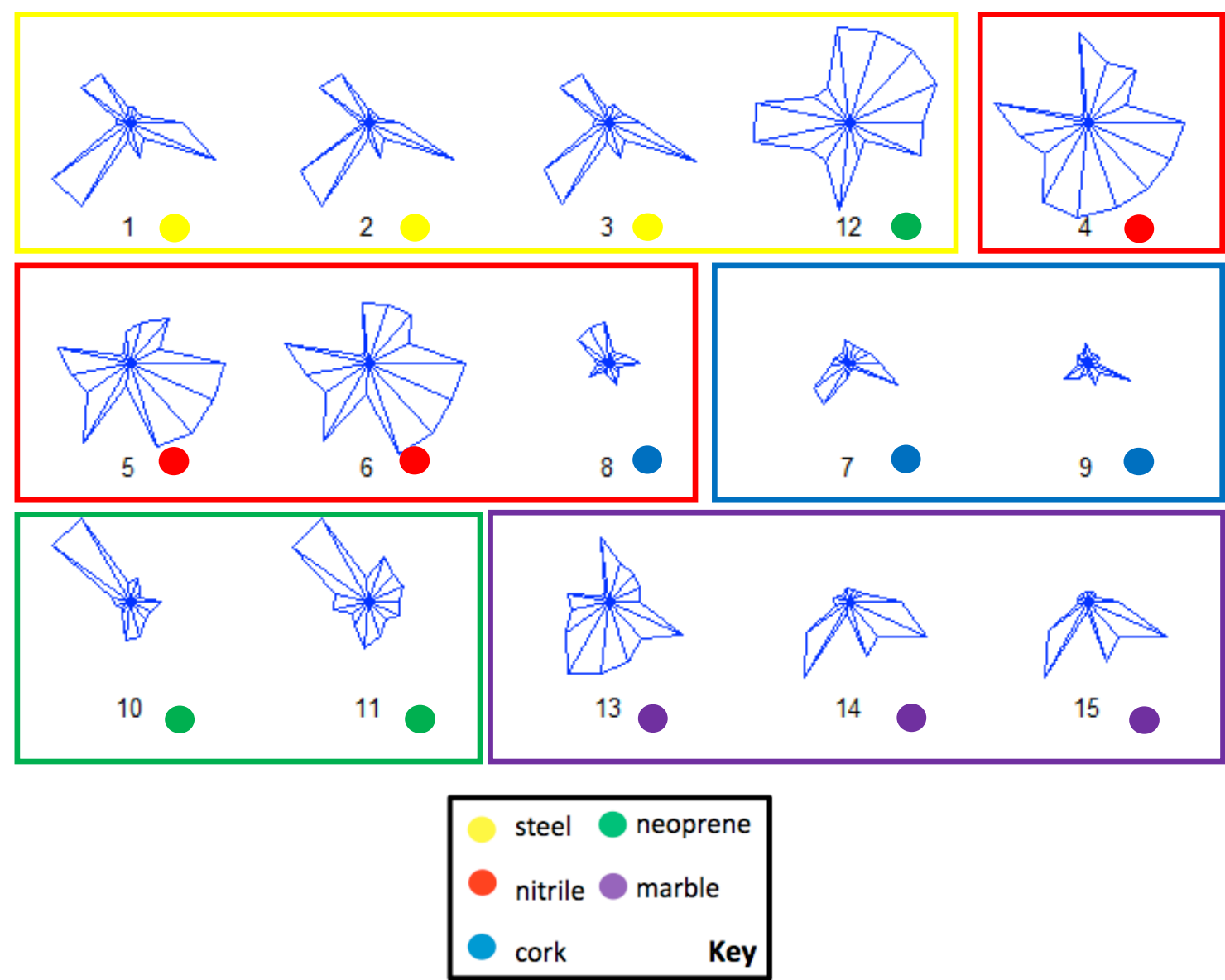

Figure 54: The 15 trials discriminated into groups based upon five acoustic characteristics acquired by the sensing transducer with grouping key 
The trials appear to be successfully discriminated with $87 \%$ accuracy; the only misclassified trials are eight and 12. Trial eight is classified in the nitrile group due to its similar rise time; trial 12 is classified in the steel group due to its similar rise time and frequency centroid. The glyph plots of the trials used for the training matrix are in figure 55.
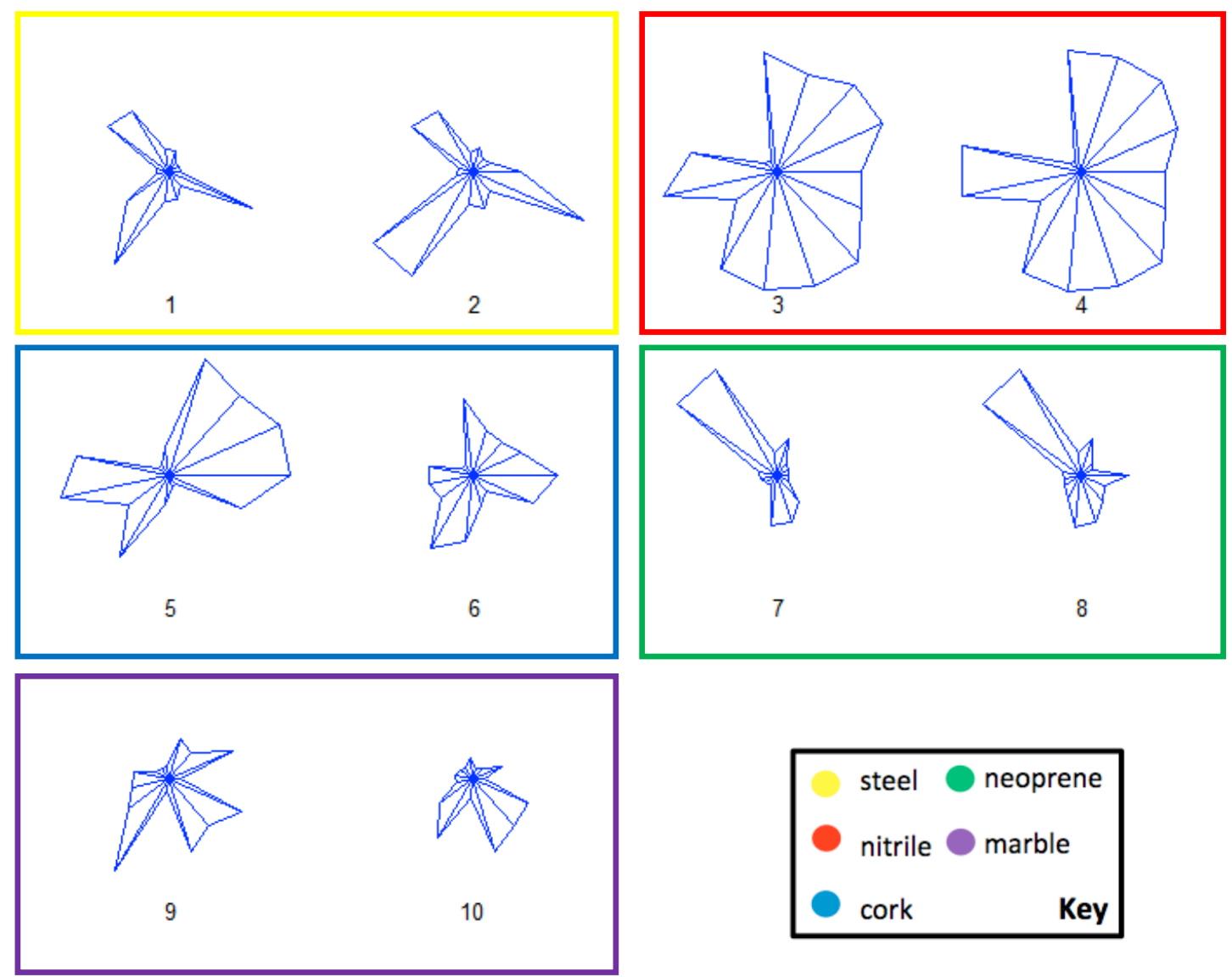

Figure 55: Glyph plots of the training matrix from which 5 characteristics are used for discrimination with grouping key

\subsection{Conclusion of the Nonlinear Waveguide Discrimination}


The different types of balls all exhibit different types of effects upon the HNSWs. The glyphs in the training group seem to match the glyphs in the corresponding discriminated groupings with about $87 \%$ accuracy. It is noted that for the cork trials in the training set, the glyphs appear to be of greater magnitude than the cork trials in the discrimination set. The accuracy of the classifier points to the fact that there may have been outliers; in particular, group 12 is dissimilar from the other neoprene glyphs.

\subsection{Waveguide Comparison}

A similar test is run on a shorter steel ball waveguide with 17 balls and on a second steel rod waveguide of the same size. Two transducers are again placed at either end of the vertically situated guides (figure 56, next page). A striker transducer excites the waveguides via a sinusoidal wave voltage waveform while a second transducer is used to collect acoustic emissions data at the opposite end of the guides. Nine different scenarios are trialed on each waveguide featuring frequencies of 50 to $150 \mathrm{kHz}$ and voltages from 2.5 to 7.5 volts. The different scenarios are compared via Fourier transform. A scenario excited at $150 \mathrm{kHz}$ and 2.5 volts is represented on the following pages for both waveguides. 


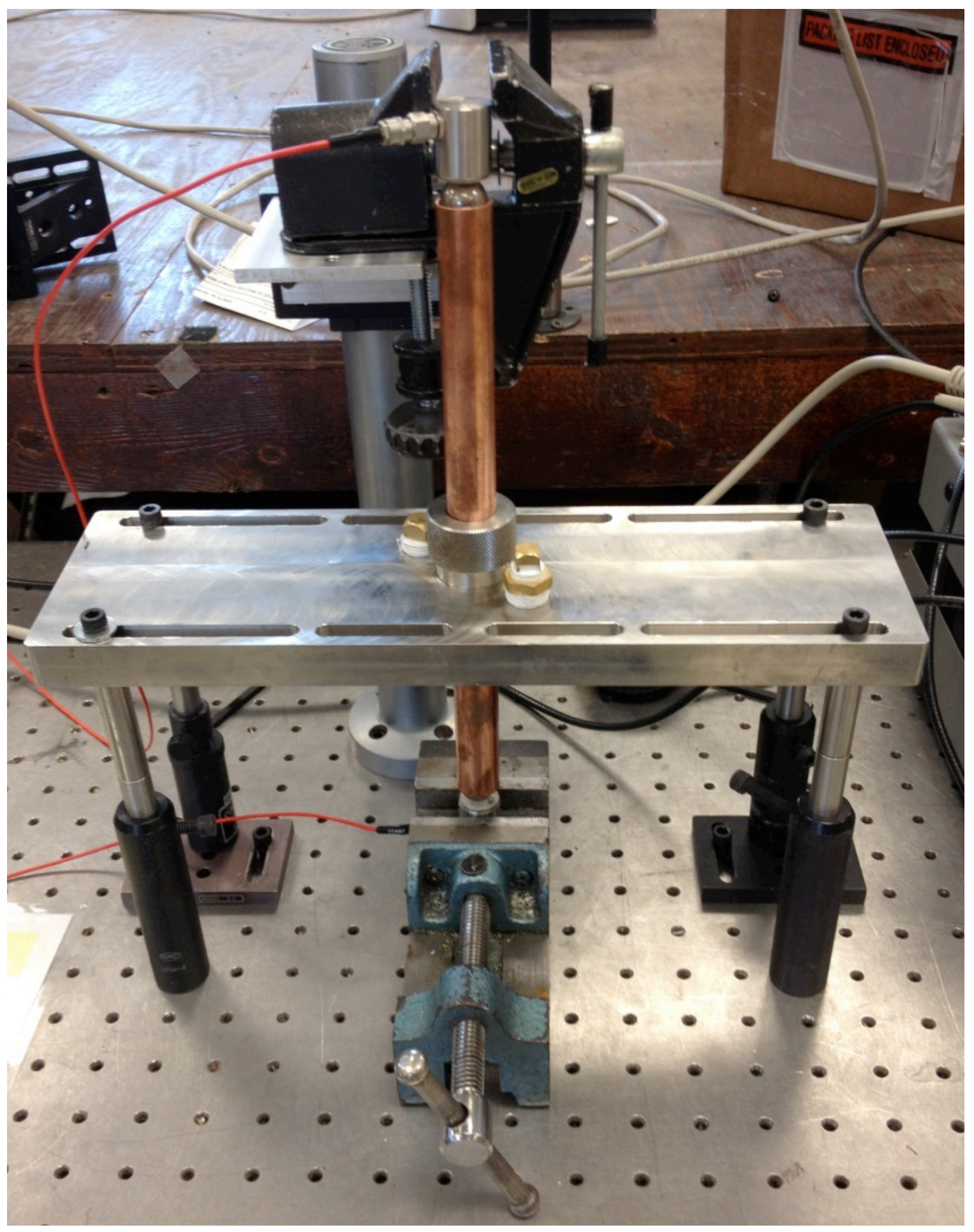

Figure 56: Steel Ball Waveguide Experiment 
FFT

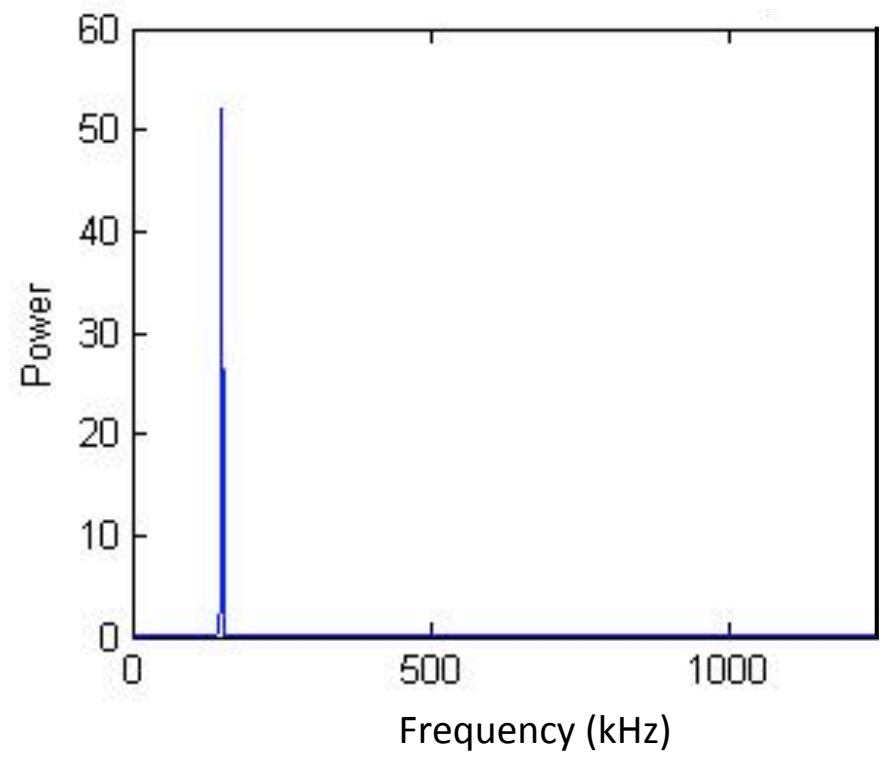

Figure 57: Fourier transform of the excitation waveform for the striker

FFT

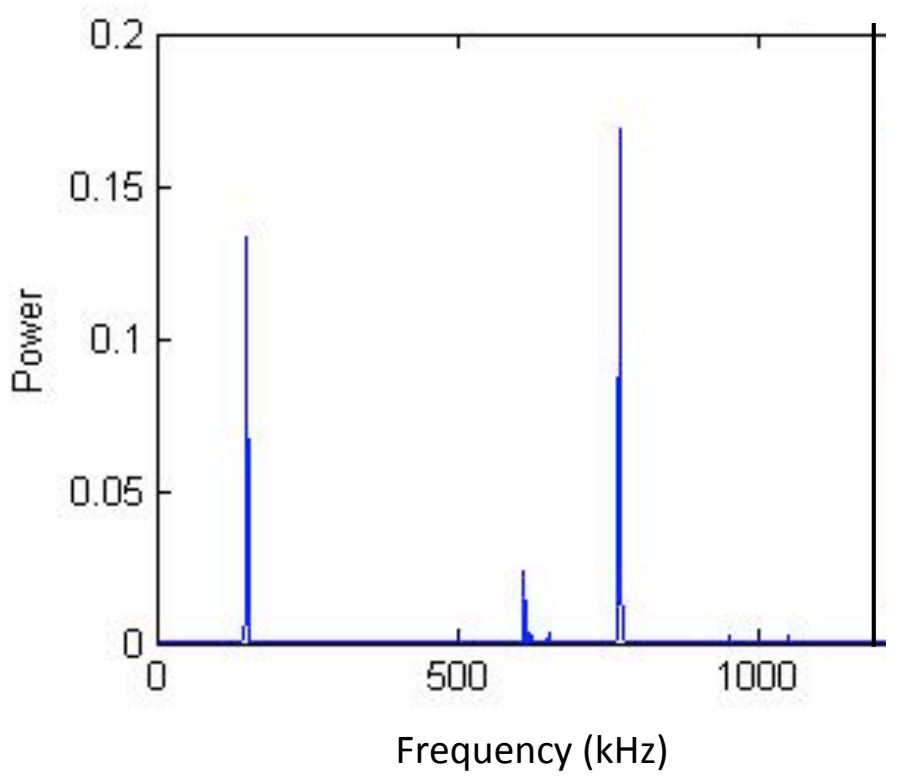

Figure 58: Fourier transform of the response from the steel ball waveguide 
Figure 57 is a Fourier transform of the striker transducer. Multiple frequency peaks found at approximately 150,600 , and $750 \mathrm{kHz}$ can be seen in figures 58 . The blip next to the center peak in figure 58 is possibly a harmonic distortion. Harmonic distortions are overtones found at integer multiples of the natural frequency that represent nonlinearities found in acoustic emissions.

\section{FFT}

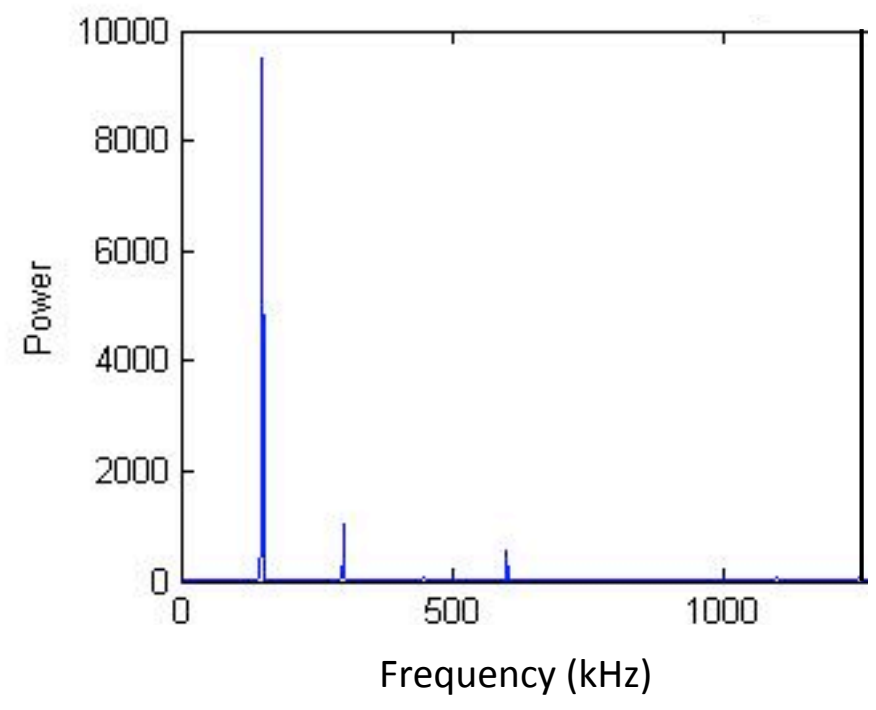

Figure 59: Fourier transform of the response of the steel rod waveguide

In figure 59, three frequency peaks are apparent at 150,300, and $600 \mathrm{kHz}$. 
Steel ball waveguides are proposed as a part of a non-destructive testing method where a transducer is used to both excite and monitor a material. The study suggests the HSNW are proficient information carriers of multiple variables and the waves are largely dependent on the elastic modulus and geometry of the material monitored. This type of waveguide could possibly be in the future of TPS sample monitoring. The Fourier transform below represents a steel ball waveguide monitoring a graphite TPS sample excited by an adjacent striker, under the same conditions as above. The frequency of response is a product of the material properties of the sample and the characteristics of the waveguide.

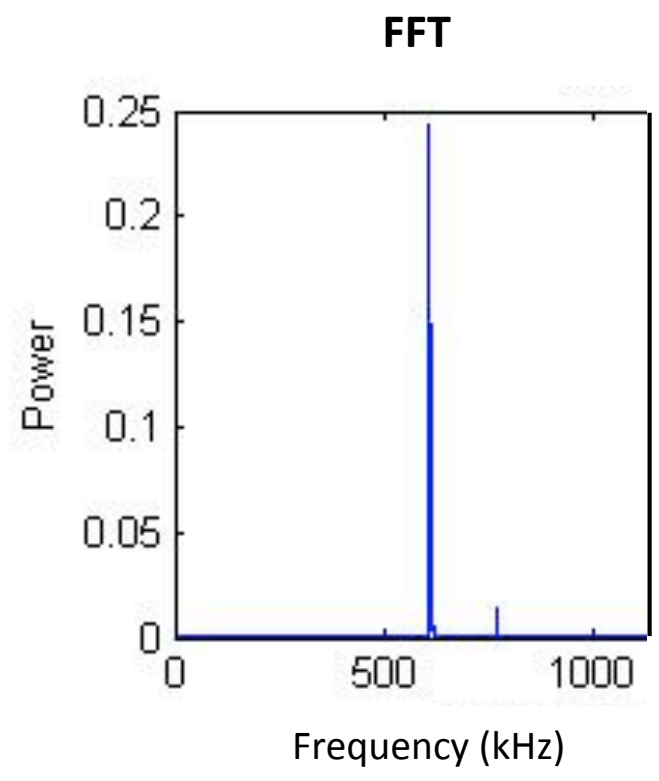

Figure 60: Fourier transform of the response of the steel ball waveguide with sample 
In figure 60 , the frequency peaks at $600 \mathrm{kHz}$ and at $750 \mathrm{kHz}$ represent the acoustic emissions of the steel ball waveguide monitoring the graphite sample. The frequency peaks occur at integer multiples of the excitation frequency. TPS sample properties and conditions can then be determined based upon this information.

The absolute mean energy response of the steel ball waveguide is $9.9 \mathrm{e}-19 \mathrm{~J}$. The absolute mean energy of the response of the steel rod waveguide is $5.11 \mathrm{e}-16 \mathrm{~J}$. The steel rod waveguide propagates waves at 50,000 strength of the steel ball waveguide. The steel ball guide propagates waves with a gain of $-25.4 \mathrm{~dB}$ compared to the striker and the steel rod waveguide propagates waves with a gain of $22.6 \mathrm{~dB}$, in the $150 \mathrm{kHz}$ frequency. 


\section{CHAPTER SEVEN: FREQUNECY SWEEPS AND PULSES}

\subsection{Function Generator Sweep}

Frequency sweeps are conducted on the three waveguides using the Agilent 33250A function generator, the Stanford Research Systems SIM983 scaling amplifier, the PAC PCI-2 board, and AEwin software. A function generator sweeps from $1 \mu \mathrm{Hz}$ to 500 $\mathrm{kHz}$ at $100 \mathrm{mV}$; the signal is fed through a scaling amplifier to create a one-volt striker amplitude. A PAC R15 $\alpha$ sensor is used as the striker while a second sensor collects data. Two steel ball waveguides of different lengths (nine balls and 17 balls), and a nine particle marble waveguide are examined. The resulting Fourier Transforms demonstrate that the steel particle waveguides of lengths nine and 17 balls produce essentially identical harmonics with the only significant variations being in the peak amplitudes.

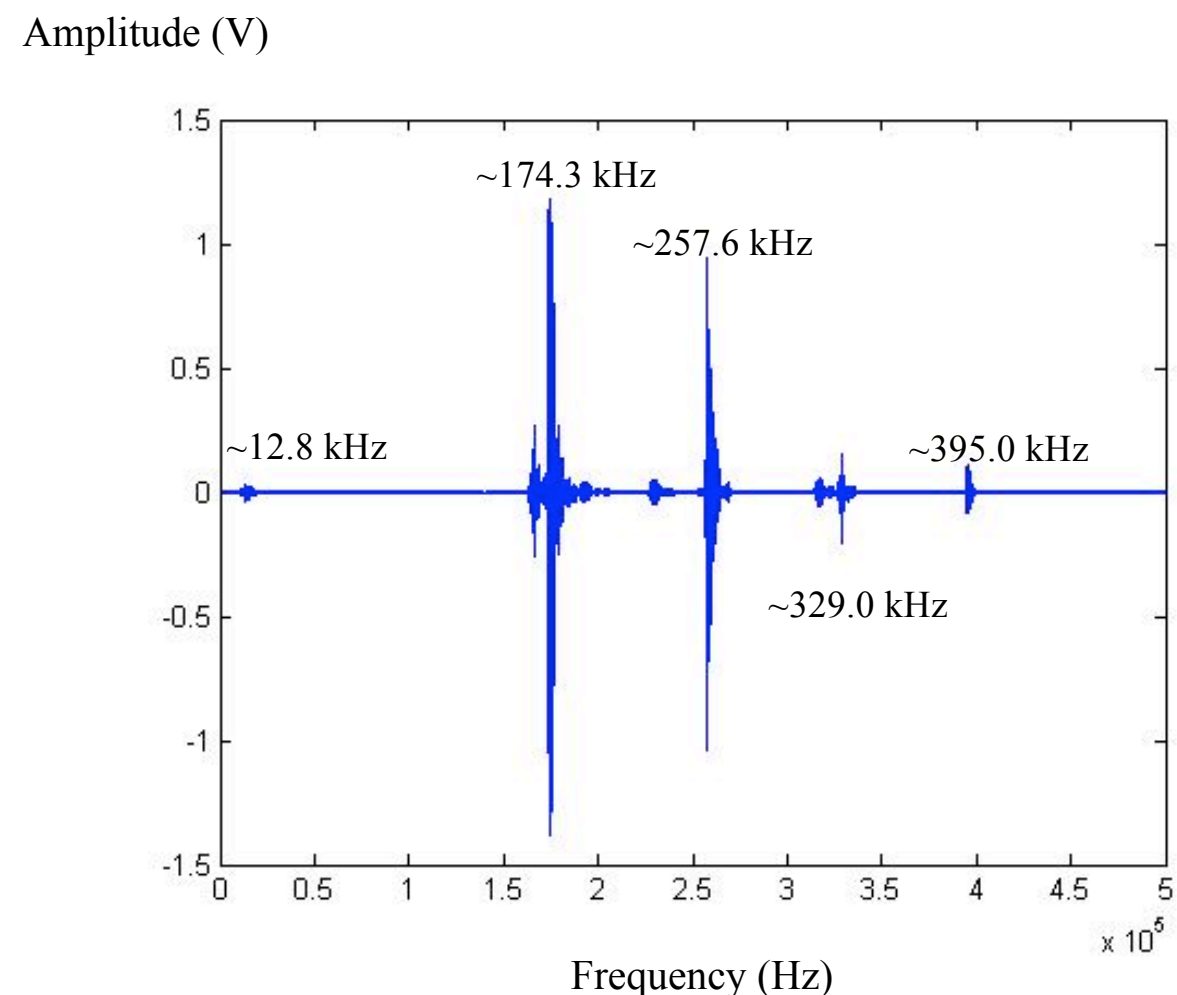

Figure 61: $1 \mu \mathrm{Hz}$ to $500 \mathrm{kHz}$ Sweep for the nine steel ball waveguide 
Amplitude (V)

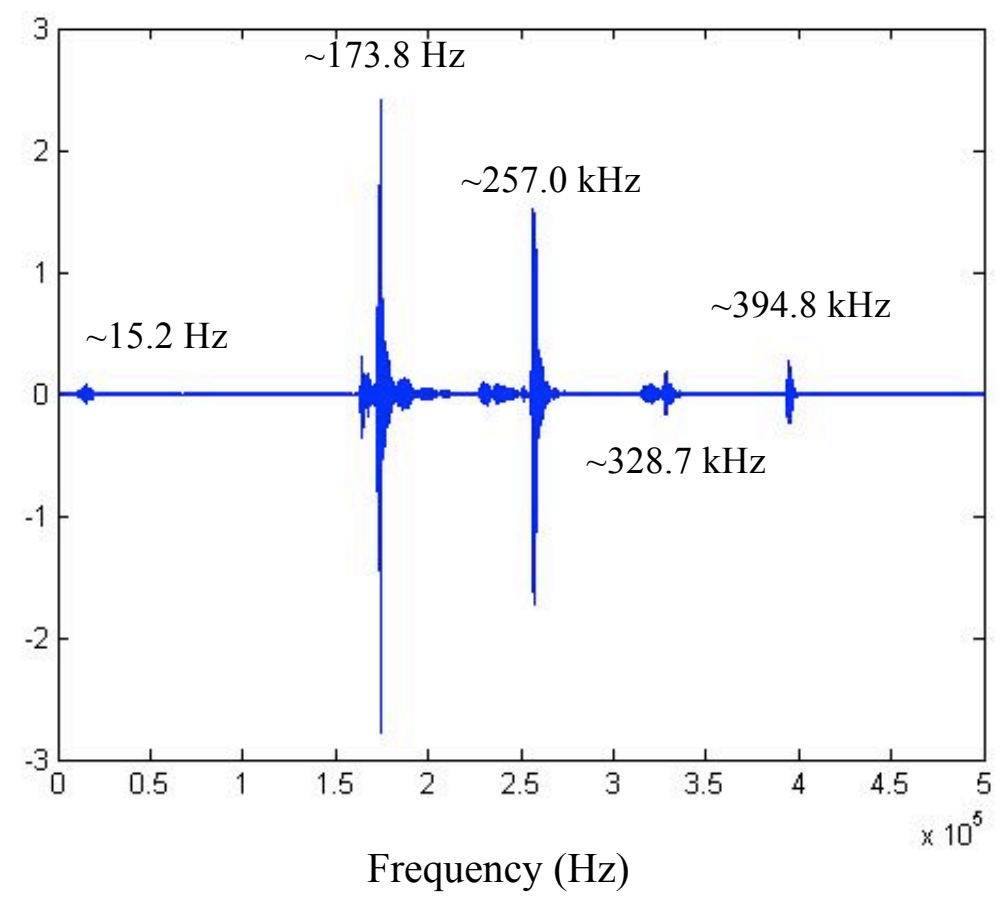

Figure 62: $1 \mu \mathrm{Hz}$ to $500 \mathrm{kHz}$ Sweep for the 17 steel ball waveguide

Amplitude (V)

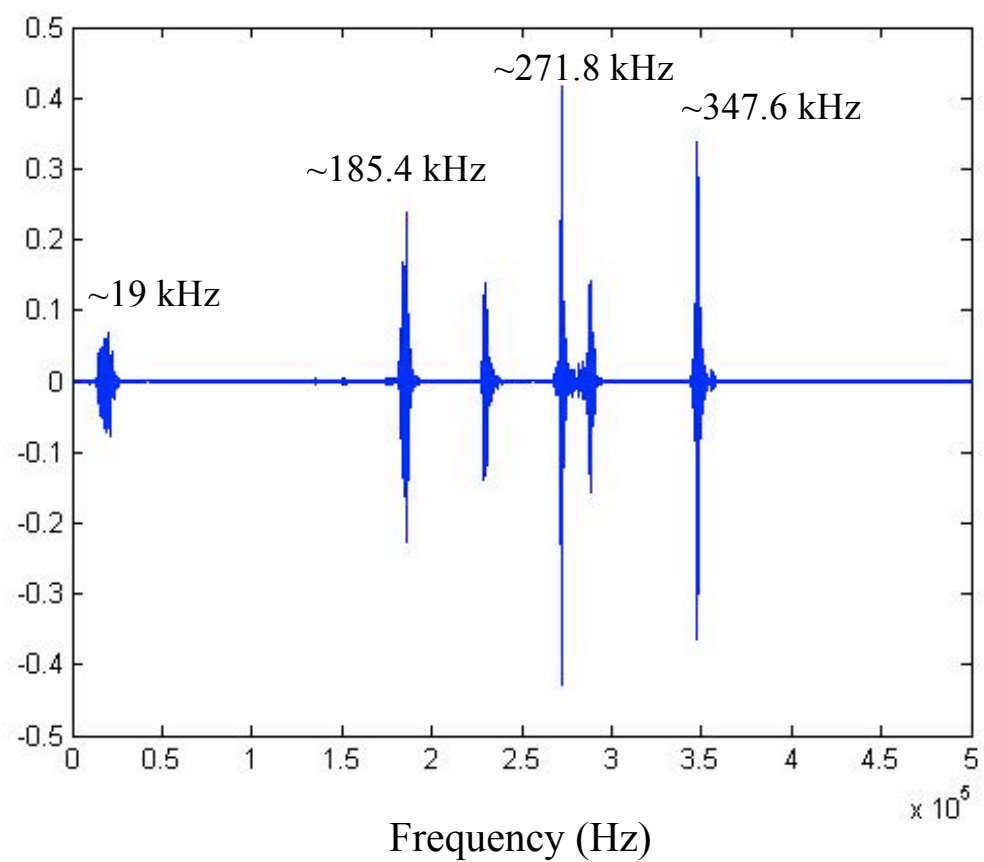

Figure 63: $1 \mu \mathrm{Hz}$ to $500 \mathrm{kHz}$ Sweep for the nine marble ball waveguide 


\subsubsection{Section Results}

The new length waveguide reveals the same harmonics at lesser amplitudes. The peak amplitude exhibited by the 9 steel ball waveguide at the $173.4 \mathrm{kHz}$ harmonic (figure 61) is approximately 2.5 times greater than the peak amplitude demonstrated by the 17 steel ball guide at this harmonic (figure 62). The peak amplitude at the $257.5 \mathrm{kHz}$ harmonic exhibited by the nine steel ball waveguide is approximately an order of magnitude greater than the absolute value of the same diminished peak amplitude exhibited by the larger guide. Changing the particle material from steel to marble (figure 63) produces a different frequency spectrum of lesser amplitudes.

A low frequency response blip is apparent at $15.2 \mathrm{kHz}$ for the nine steel ball guide and $12.8 \mathrm{kHz}$ for the 17 steel ball guide; these low frequency response blips are lesser than the neighboring higher frequency peaks at merely $37 \mathrm{mV}$; the lower frequency visible responses of the waveguides are possibly evidence of the slow moving wave characteristic to these guides. The remaining frequency spectrum seems to again behave independently of waveguide length, at least for these two practical sized waveguides. The only difference in the spectra is the diminished amplitudes at the peaks product of increased waveguide length due to greater frictional losses of the longer waveguide. Shorter waveguides seem to produce greater amplitude frequency spectra. 


\subsection{Frequency Sweeps Under Precompression}

The same experiment from 7.1 is conducted with a six-ball waveguide in the vertical position and under precompression to investigate the first frequency blip, results in figures 64 (below) and 65 (next page).

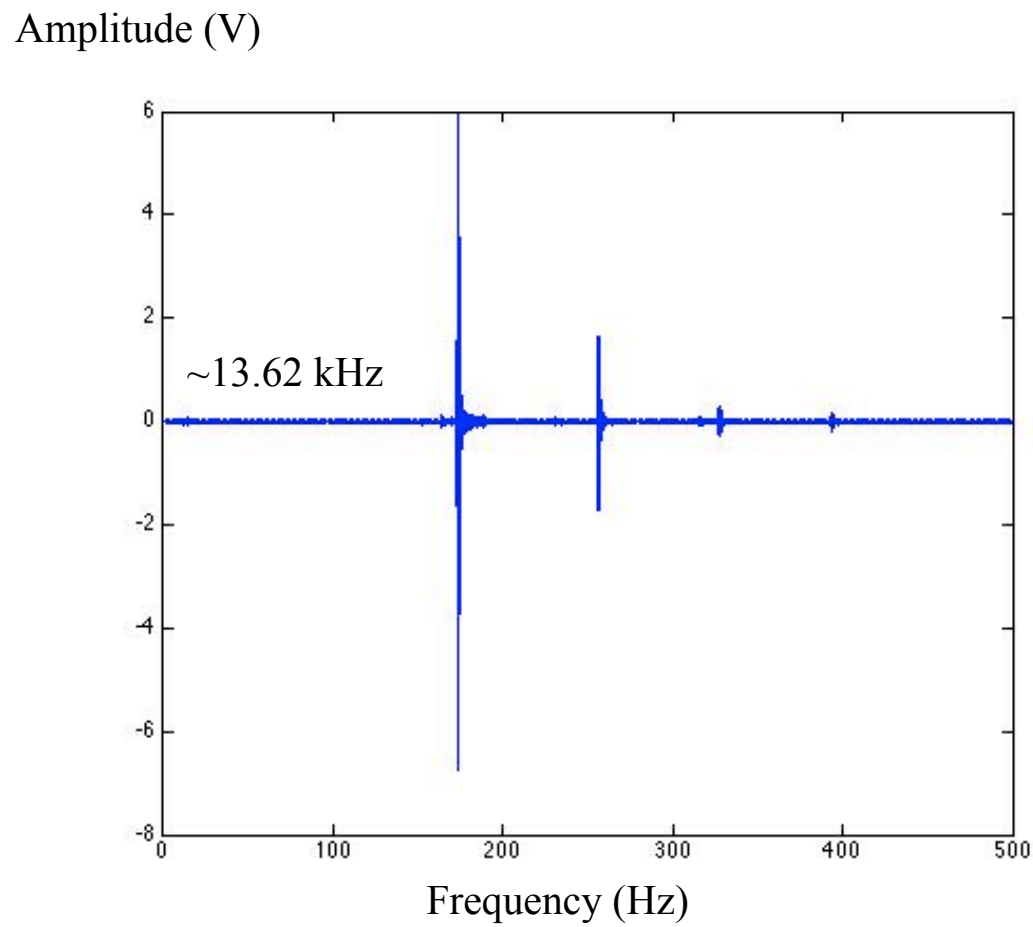

Figure 64: $1 \mu \mathrm{Hz}$ to $500 \mathrm{kHz}$ Sweep for the six steel ball waveguide with $1.28 \mathrm{~kg}$ precompression 
Amplitude (V)

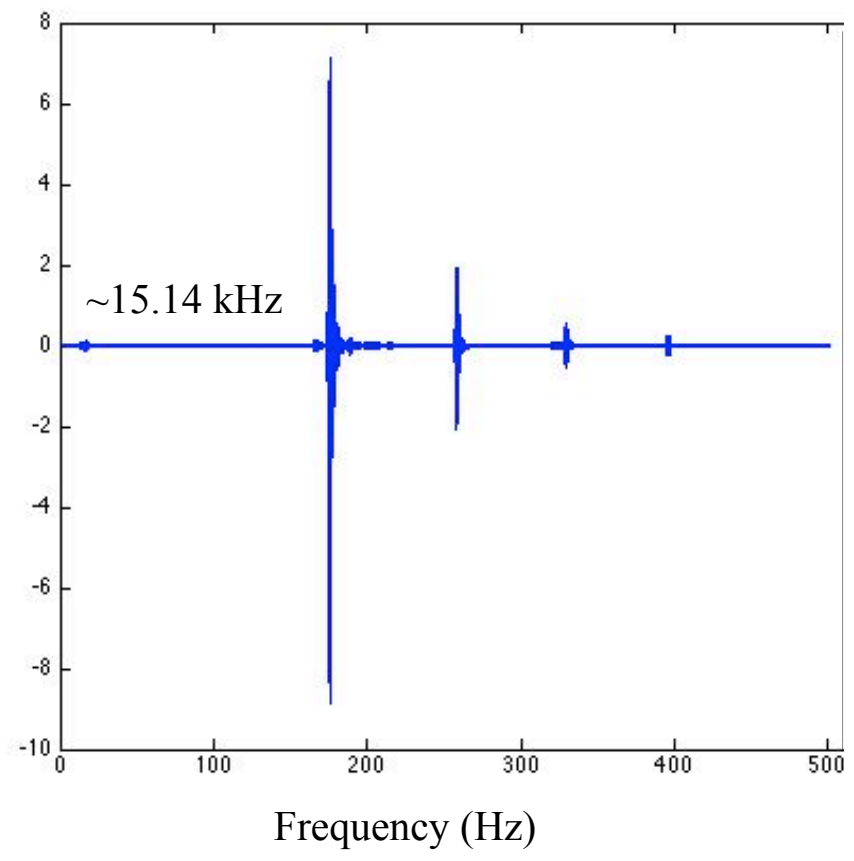

Figure 65: $1 \mu \mathrm{Hz}$ to $500 \mathrm{kHz}$ Sweep for the six steel ball waveguide with $3.28 \mathrm{~kg}$ precompression

\subsubsection{Section Results}

When changing the length of a waveguide, particle material, or by adding precompression to a waveguide, the first frequency blip visible in the sweeps from this chapter changes in frequency and amplitude. Adding precompression to a waveguide increases the frequency and amplitude of the slow moving solitary wave characteristic to these waveguides.

\subsection{Variation in Pulse Width}

A nine steel ball copper tube waveguide is positioned vertically with one transducer at either end. The function generator and scaling amplifier are used to power a "striker" that creates an acoustic pulse with piezoelectrics. The width of this pulse is 
varied while the second sensing transducer acquires a signal. The frequency of the pulse is aligned with the first harmonic of the waveguide at approximately $173.24 \mathrm{kHz}$. The pulse width is varied from four microseconds to one microsecond. A Fourier transform of each of the four variations are found in figures 66-69 below and on the following page. The four frequency spectra all appear to bear similar frequency peaks with greater amplitudes corresponding to larger pulse widths. There are slight differences noticeable between the spectra in the ranges from 200 to $250 \mathrm{kHz}$ and from 450 to $750 \mathrm{kHz}$.

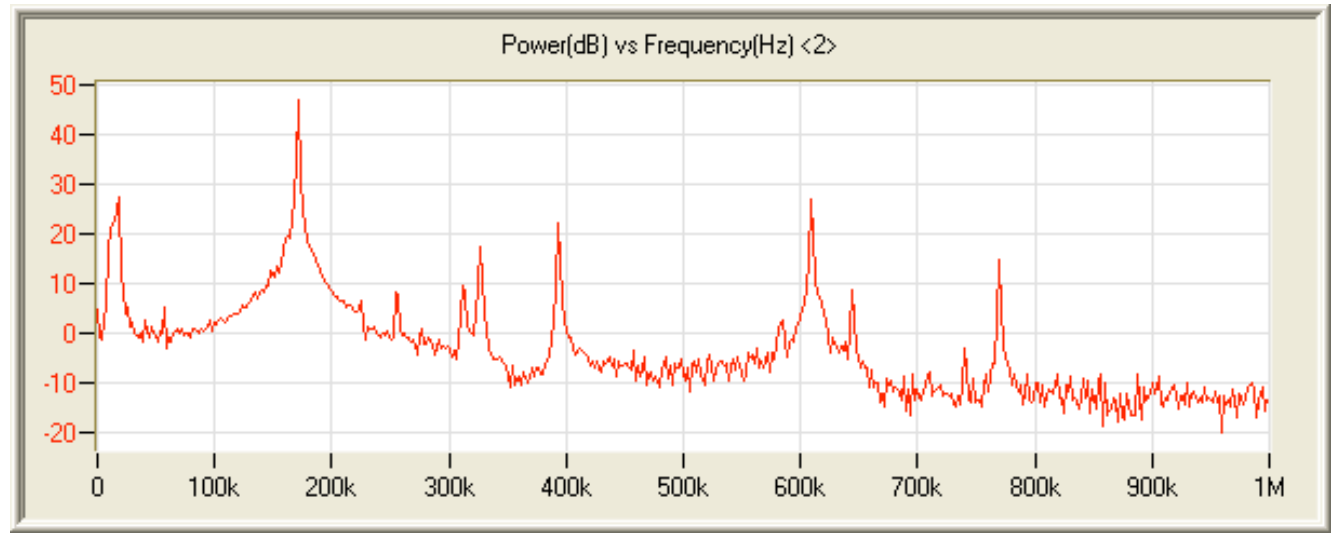

66: pulse with four-microsecond width

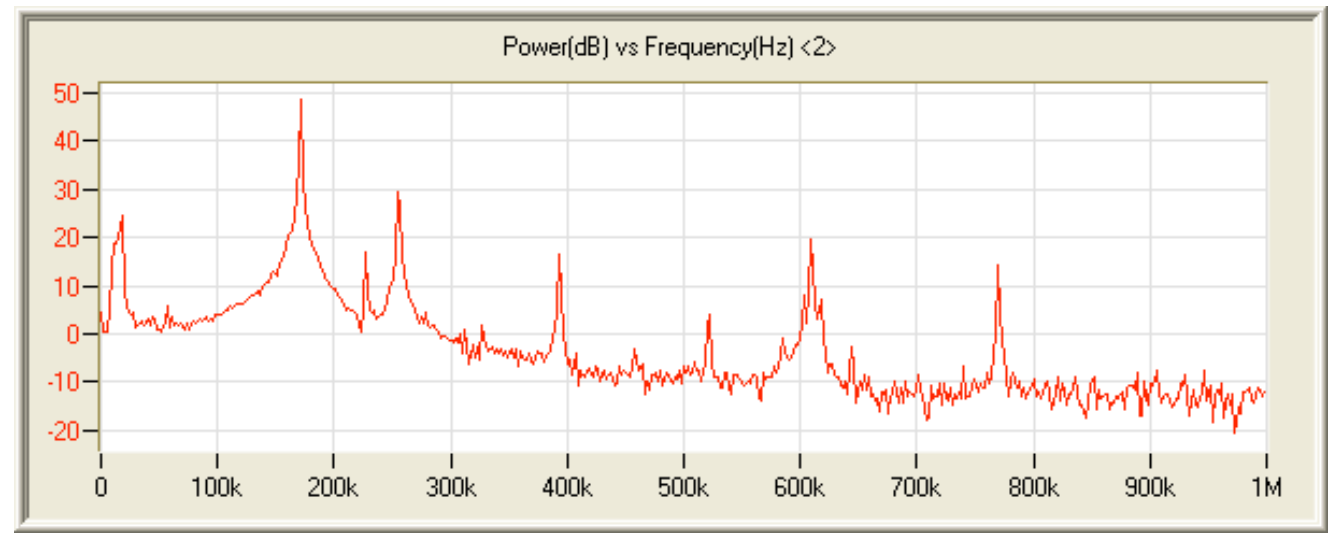

Figure 67: pulse with three-microsecond width 


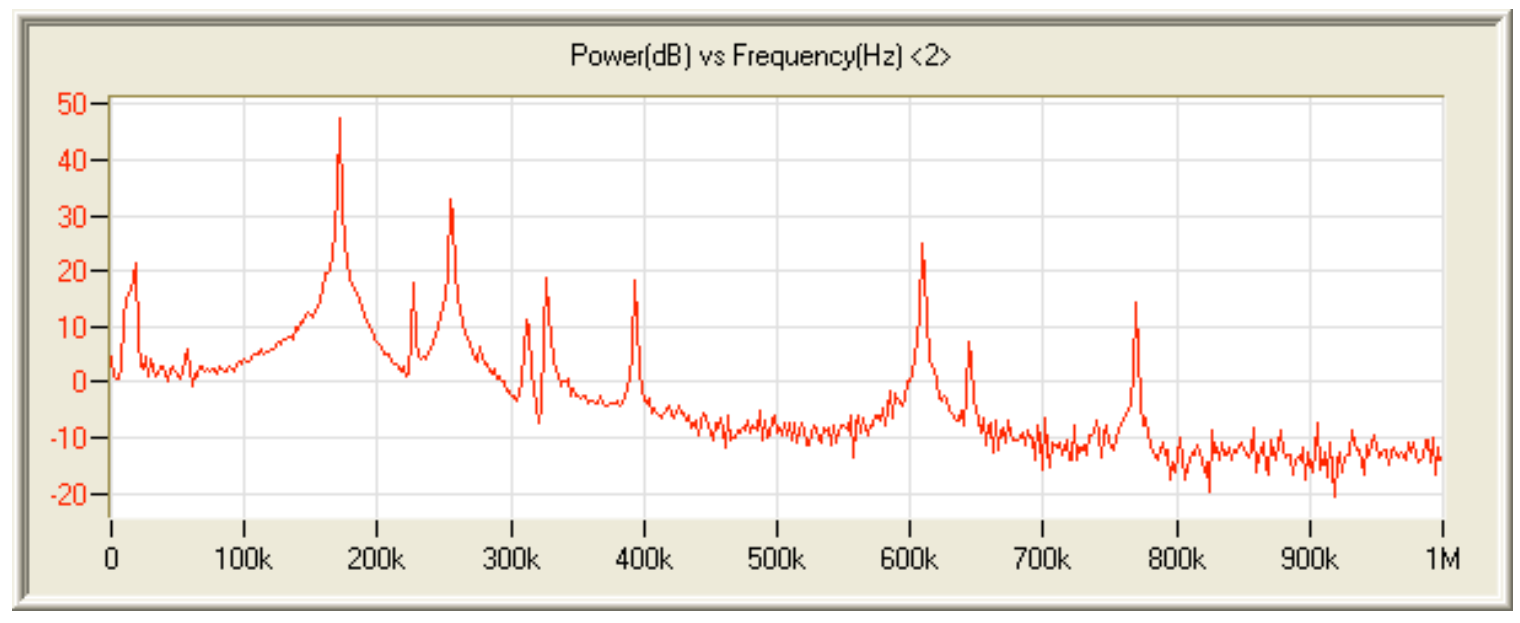

Figure 68: pulse with two-microsecond width

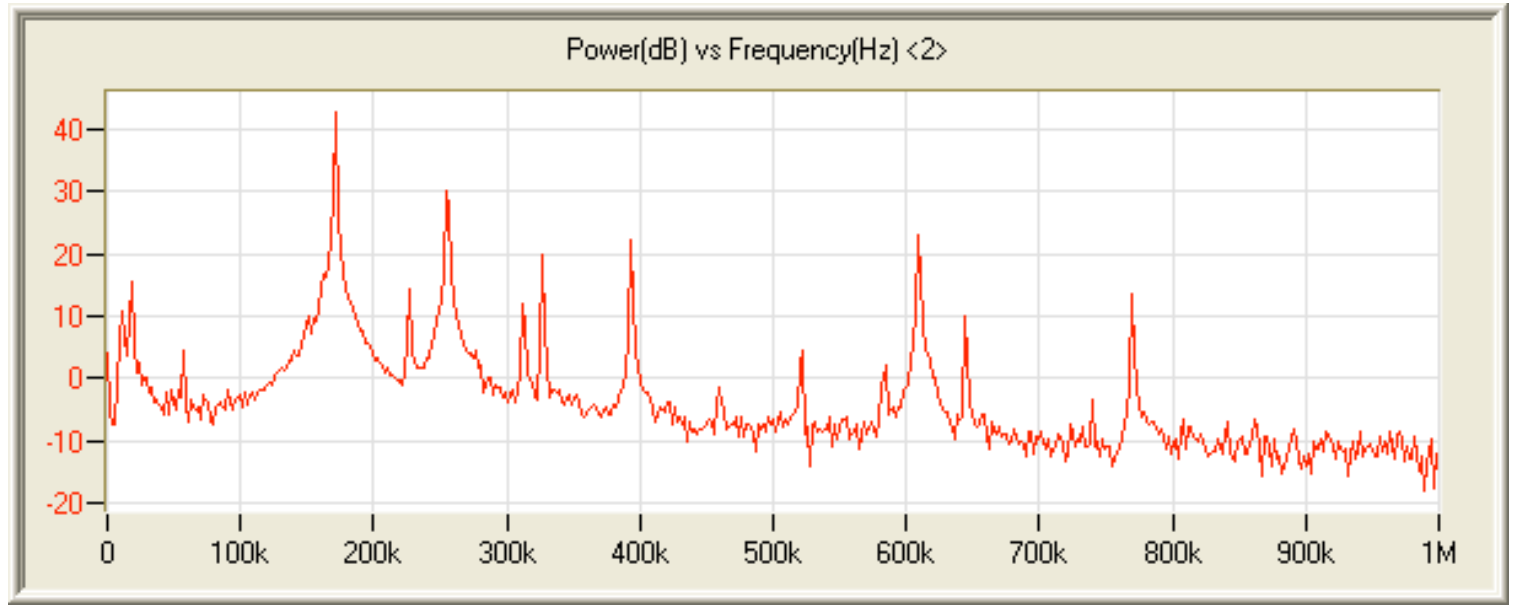

Figure 69: pulse with one-microsecond width

\subsubsection{Section Results}

A shorter pulse can better excite higher frequency in a steel ball waveguide.

Shorter pulses also create more peak frequencies visible in the power spectrum above (figure 69) from the response a steel ball waveguide. With a shorter pulse the peak frequencies in the power spectrum become more apparent. 


\section{CHAPTER EIGHT: DEFORMATION}

\subsection{Deformation of a single steel ball}

This chapter portrays the deformation experienced by a steel ball lodged in between two granite surfaces such as the steel balls in a steel ball waveguide. This is a depiction of what exactly is happening to the steel balls inside of the steel ball waveguide as a wave is propagating. This also serves as a depiction of what the steel balls experience as the waveguide is put into a precompression.

The deformation of a single steel ball lodged in between two granite faces is calculated using COMSOL. One of the faces is held fixed while a small force is applied to the second face pinching the ball in between the two. In this simulation the applied force is $0.452 \mathrm{mN}$, an estimate of the peak force applied to the ball by the striking transducer. The following figure approximates the peak displacement experienced by one of the steel balls in a waveguide upon enduring sinusoidal excitations via transducer of one volt at $174 \mathrm{kHz}$. The maximum displacement location occurs furthest from the contact points with the granite with a peak maximum displacement of $2.313 \mu \mathrm{m}$ (figure 70, next page). 
A $2.3132 \times 10^{-3}$

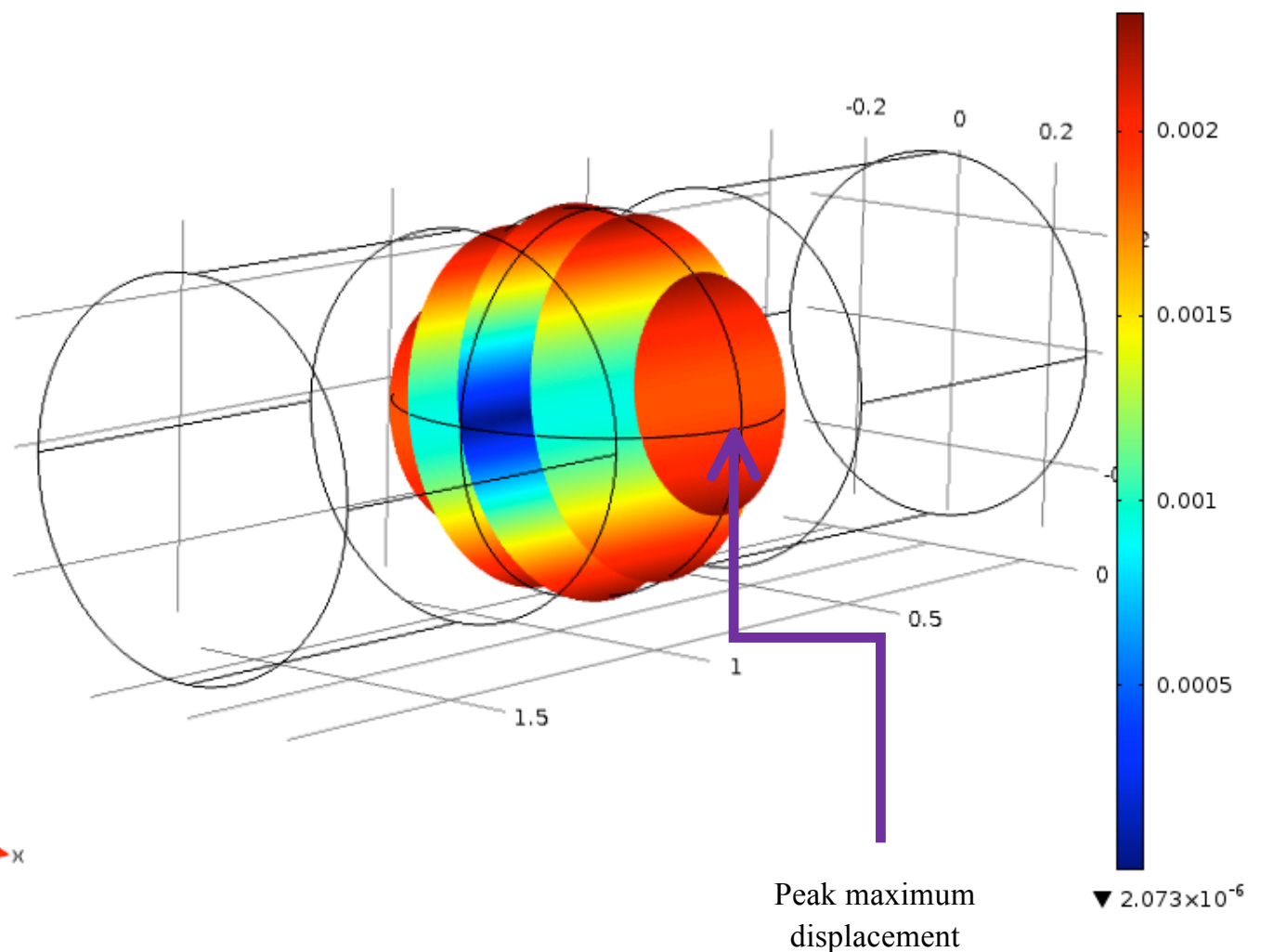

Figure 70: Peak deformation gradient of a steel ball during sinusoidal excitation 


\section{CHAPTER NINE: FORCE SENSOR EXPERIMENTS}

\subsection{Steel Ball Waveguide}

\subsubsection{Test Setup}

An experimental test bed was developed to assess the performance of a ball chain waveguide with the ultimate goal being to ascertain and eventually fine tune the capabilities for acoustic emission testing with thermal protection systems. Elastodynamic waves are transmitted through a steel ball waveguide placed between transducers in a test set up; the wave is monitored at two different points using custom fabricated $5 / 8$ " diameter balls containing embedded piezoelectric elements [9]. Upon initial testing it was determined that the piezoelectric sensors are not sensitive enough to pick up the motion of the piezoelectric transducer and thus a striker ball is used to excite the chain in order to report motion at the sensor locations. The six-ball dimer chain is positioned vertically in a stand and accompanied by a loading ram that provides axial precompression. A piezoelectric disc sensor is placed in the center of the six balls inside the copper tube waveguide with an orifice in the wall allowing the signal of the sensor to be measured by a data acquisition unit. A second sensor is fixed within a steel ball and placed at the end of the waveguide (figure 71). Liquid tape is used to cover the sensors so they do not break during testing and to seal the second sensor within the steel ball. 


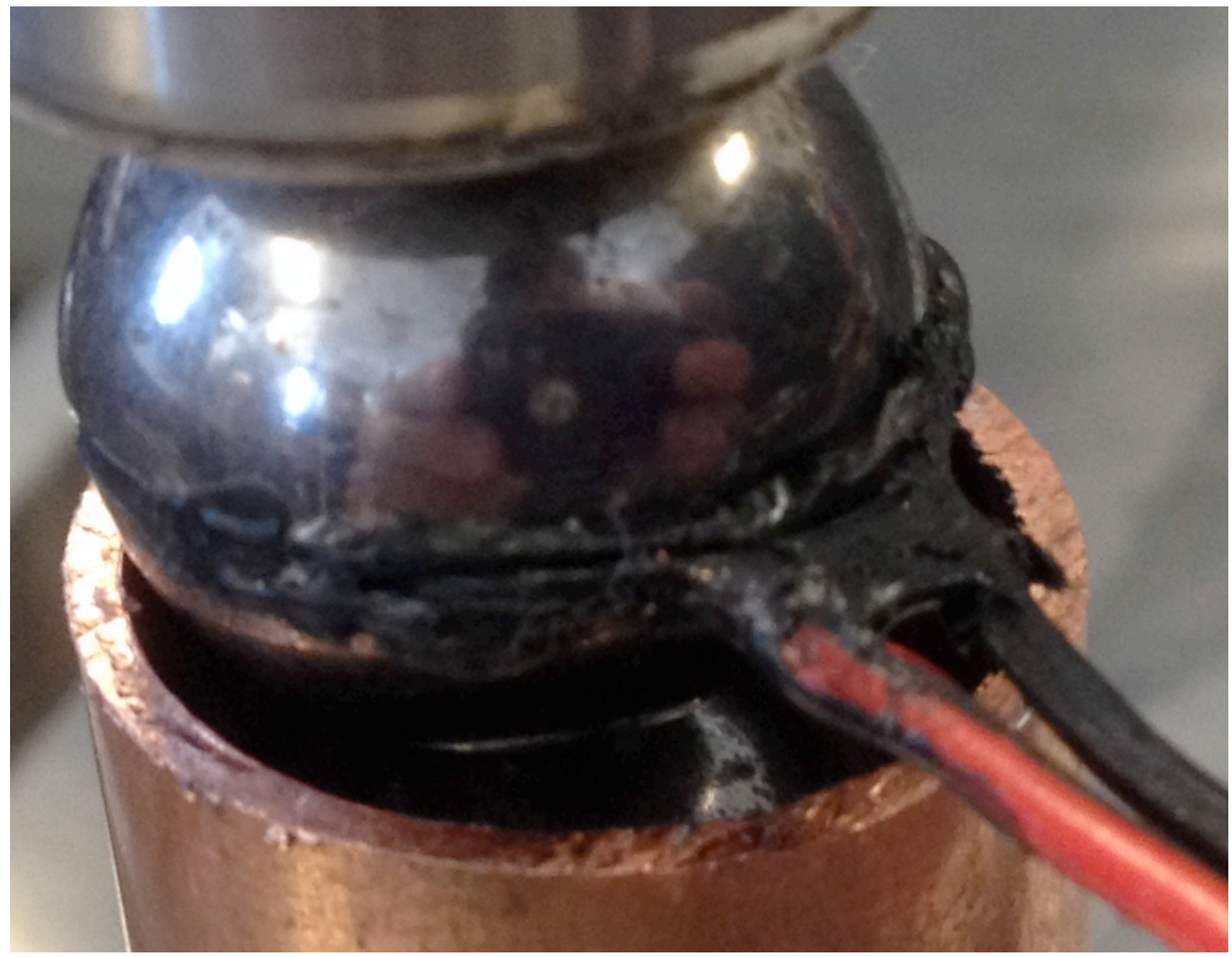

Figure 71: Close up of a piezoelectric sensor fixed within a steel ball

A steel ball is dropped from two locations upon the head of the precompression component (figure 72); the ball is dropped from approximately $100 \mathrm{~mm}$ above the unit and $200 \mathrm{~mm}$ above the unit. The trials are compared and resulting Fourier transforms of the two sensors are analyzed. 


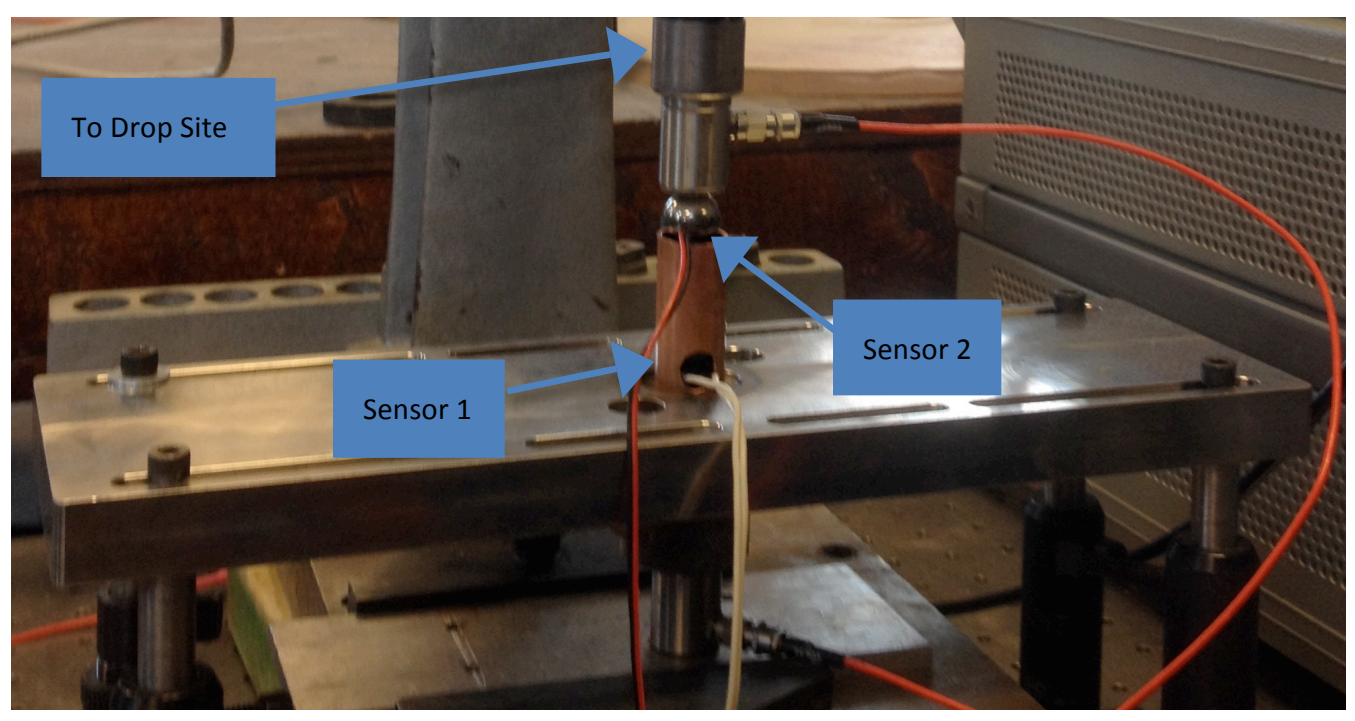

Figure 72: Close up of the testing rig with sensor labels

\subsubsection{Section Results}
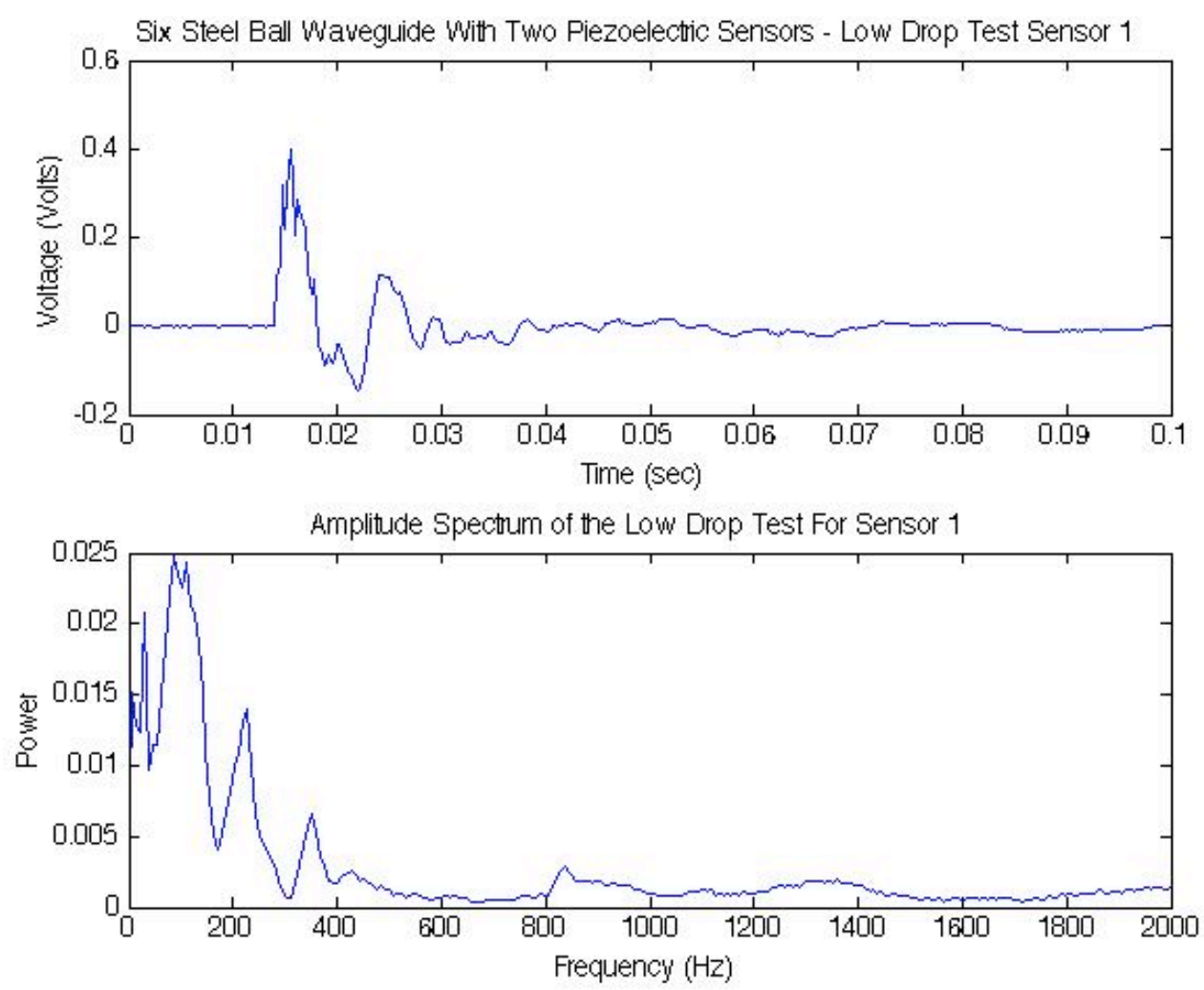

Figure 73: Results of the low drop test from piezoelectric sensor 1 
Six Steel Ball Waveguide With Two Piezoelectic Sensors - Low Drop Test Sensor 2
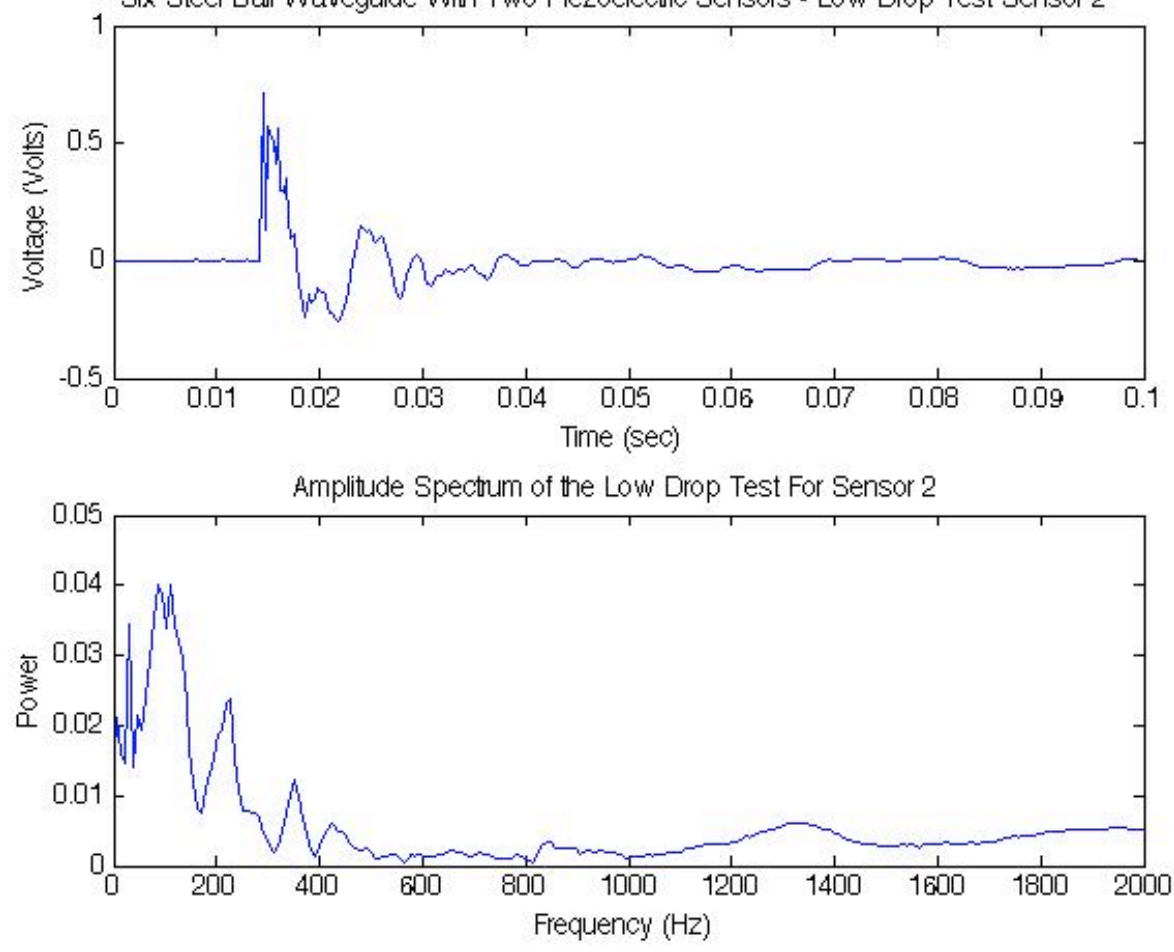

Figure 74: Results of the low drop test from piezoelectric sensor 2
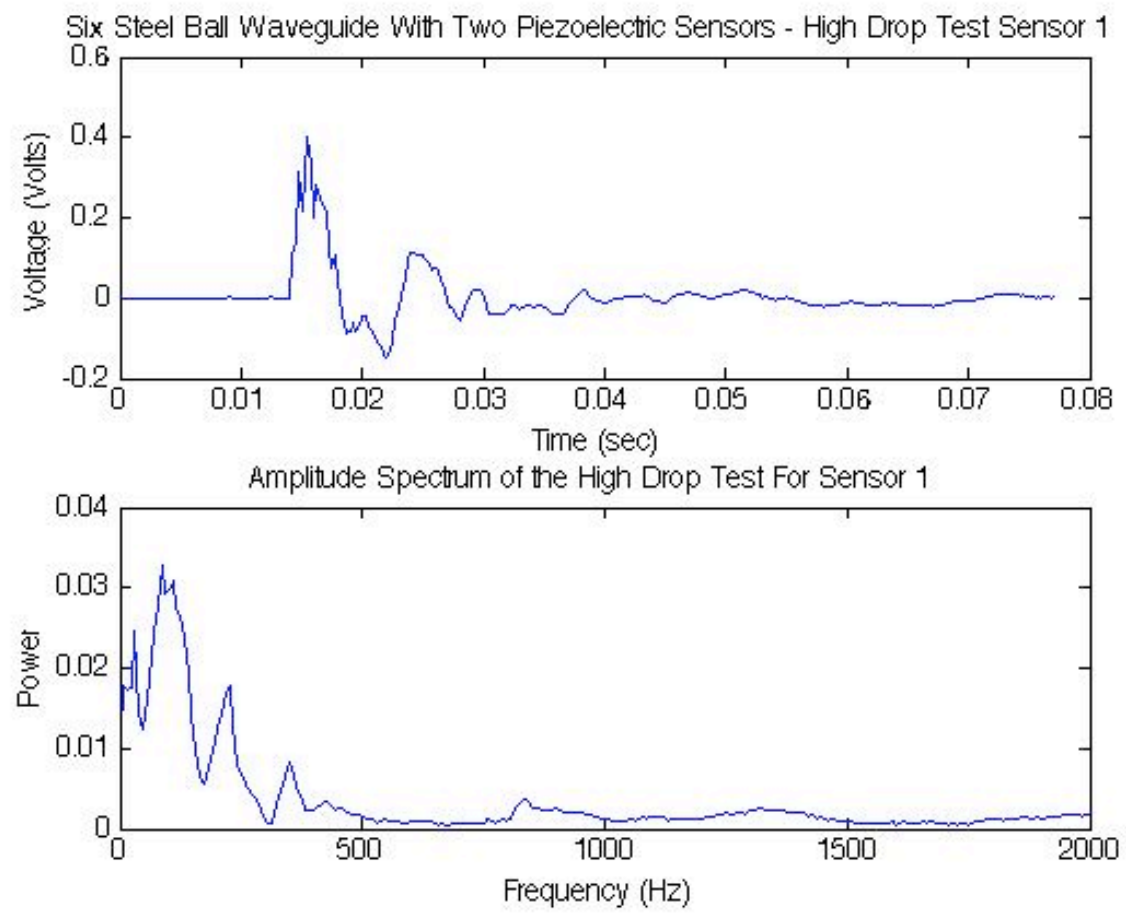

Figure 75: Results of the high drop test from piezoelectric sensor 1 

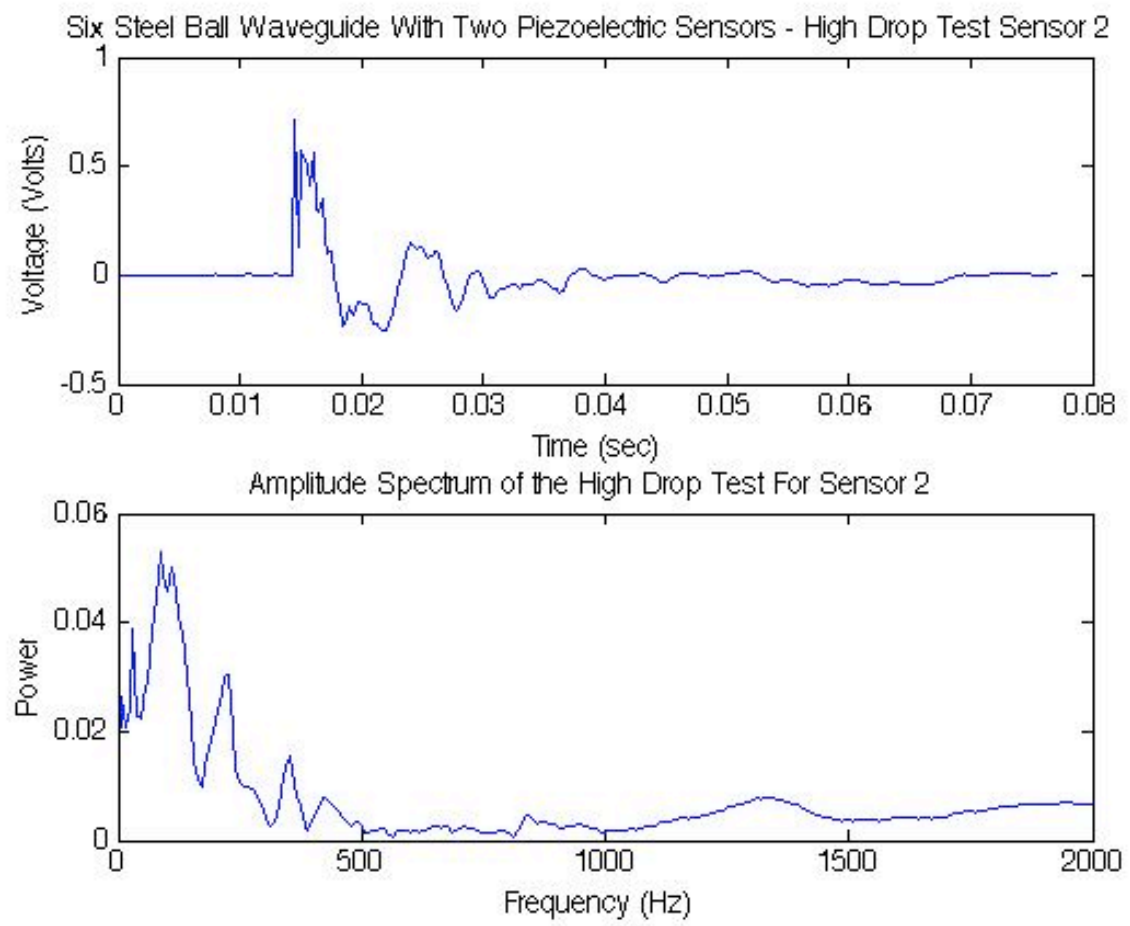

Figure 76: Results of the high drop test from piezoelectric sensor 2

Six Steel Ball Waveguide With Two Piezoelectic Sensors - Low Drop Test Sensor 1

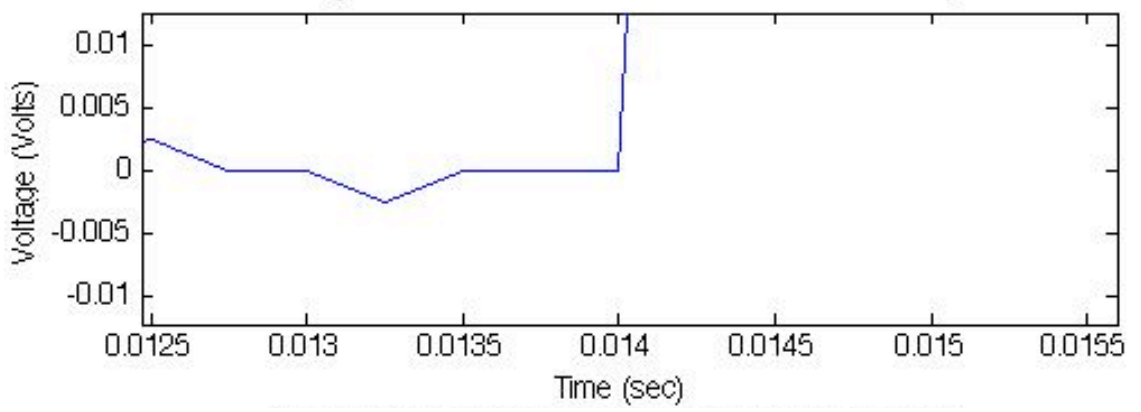

Six Steel Ball Waveguide With Two Piezoelectic Sensors - Low Drop Test Sensor 2

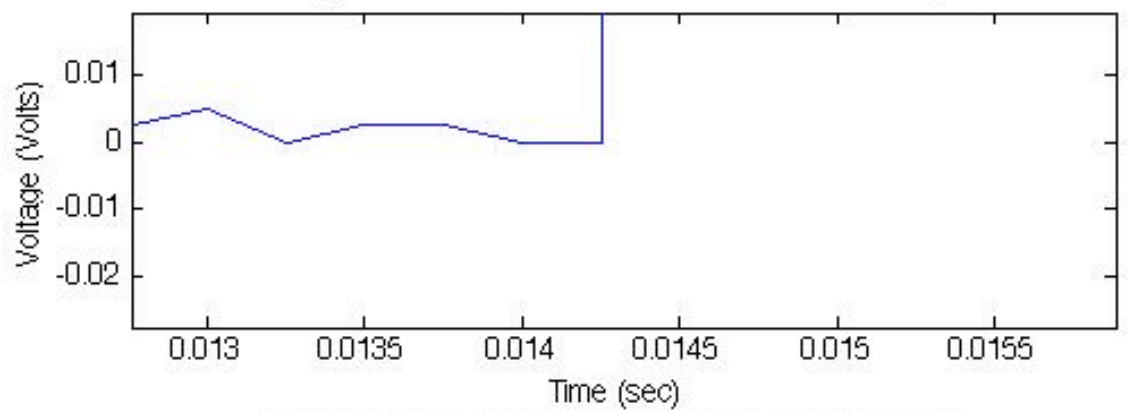

Figure 77: Side by side comparison of the trigger time of sensor 1 and sensor 2 from the low drop test 
Results can be seen in figures 73-77. The mean peak voltage of the two sensors from the high drop test is greater than the mean peak voltage of the two sensor from the low drop test indicating that the tests where successful. During the test the dropped ball bounces upon the drop site, which is visible in the voltage vs. time plots from both sensors in both trials. The Fourier transforms from both sensors in both trials are all fairly similar with dominant frequency peaks at 50, 120, 220, and $370 \mathrm{~Hz}$.

\subsubsection{Analysis}

Sensor one is triggered slightly before sensor two; this is unnoticeable in the plots due to the small interval of time between the triggers. The trigger time difference between sensor one and sensor two for the low drop test is approximately $25 \mu$ s. The trigger time difference between sensor one and sensor two for the high drop test is the same. The wave speeds of the two waves can be calculated with equation 19:

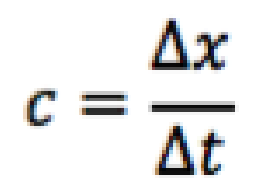

\section{Equation 19: Wave speed}

The distance between the two sensors is approximately $3.96 \mathrm{~cm}$. The wave speed of the wave from the low drop test is calculated to be approximately $158.5 \mathrm{~m} / \mathrm{s}$; the wave speed of the wave from the high drop test is equivalent. The force sensors are calibrated and measure $171 \mathrm{~g} / \mathrm{V}$. With this calculation applied to equation 15 , the theoretical wave velocity of the low drop test is $228.0 \mathrm{~m} / \mathrm{s}$ and the theoretical velocity of the high drop test 
is calculated to be the same. Similarly a pencil lead test is performed upon the six-ball waveguide with only one central sensor. The force sensor results appear in figure 78 .

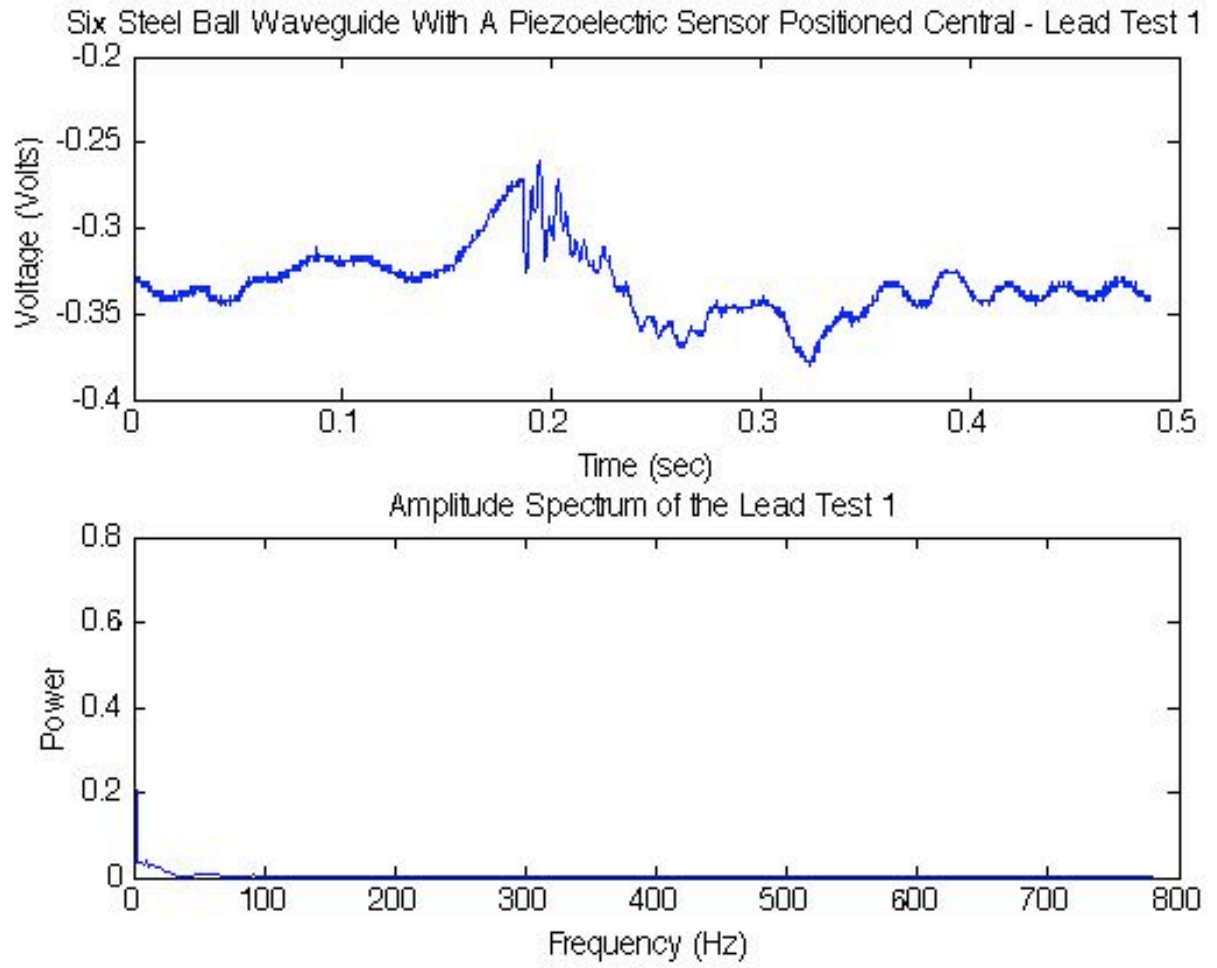

Figure 78: Results of the pencil lead test with one central sensor

\subsection{Steel Rod Waveguide}

\subsubsection{Test Setup}

Two piezoelectric disc sensors are placed in a steel rod waveguide the same length as the steel ball waveguide from 9.1. Liquid tape is again used to cover the sensors so they do not break during testing. The waveguide is placed in the same test-bed from 9.1 (figure 79, next page). 
A steel ball is again dropped from the same two locations upon the head of the precompression component as from 9.1. The trials are again compared and resulting Fourier transforms of the two sensors are analyzed.

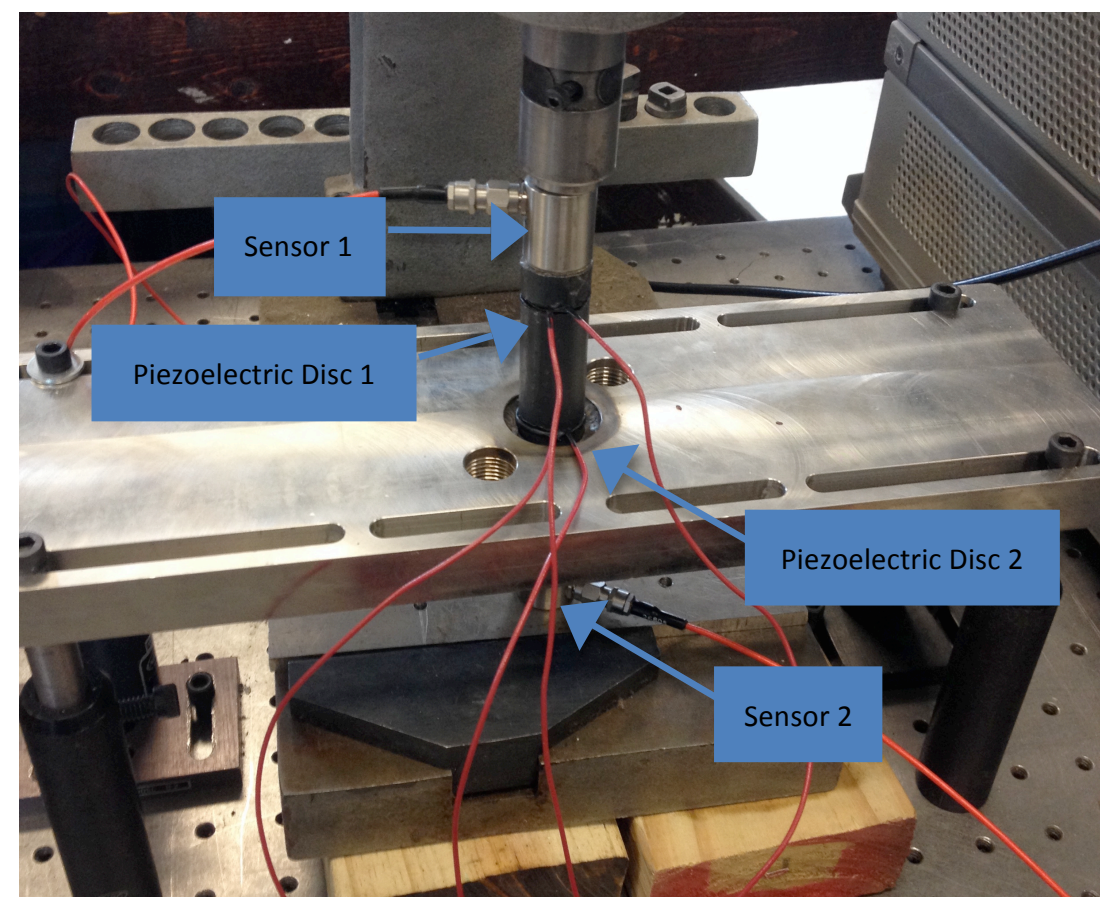

Figure 79: Close up of the steel rod in the testing rig 


\subsubsection{Section Results}
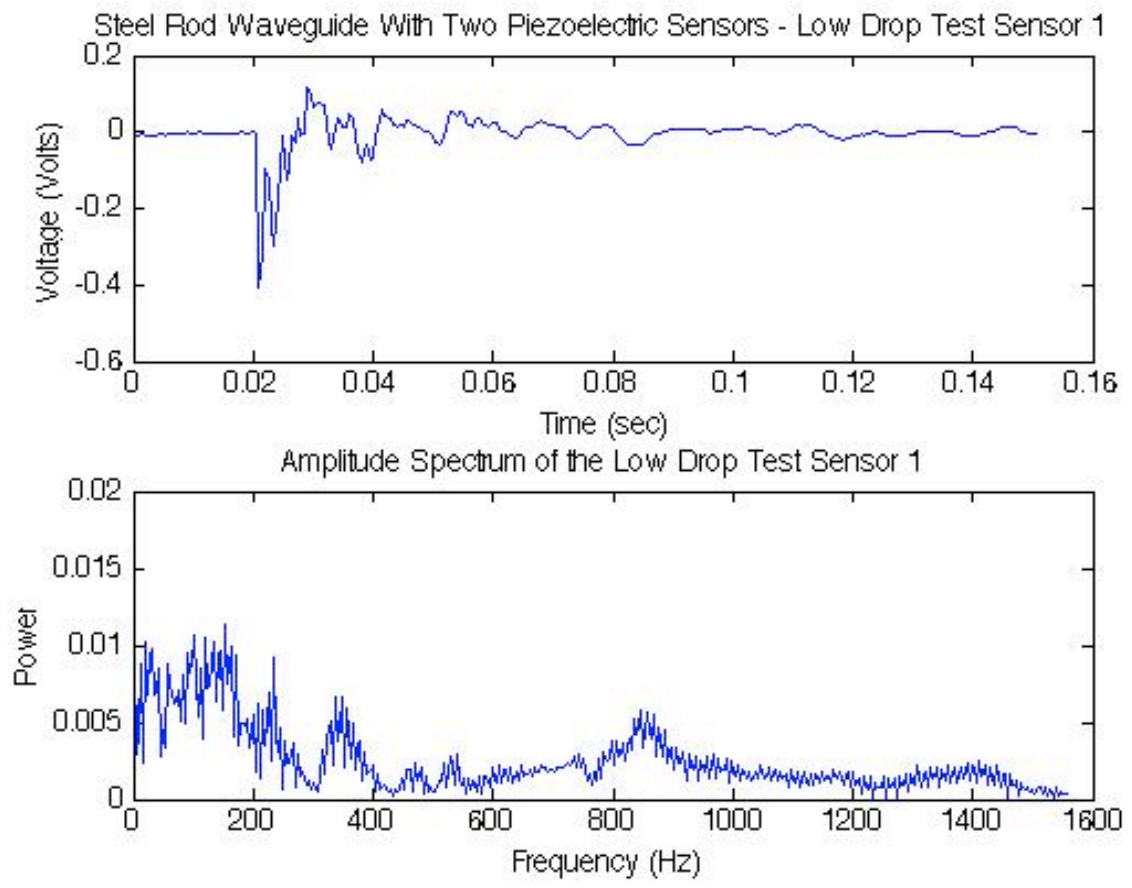

Figure 80: Results of the low drop test from piezoelectric sensor 1
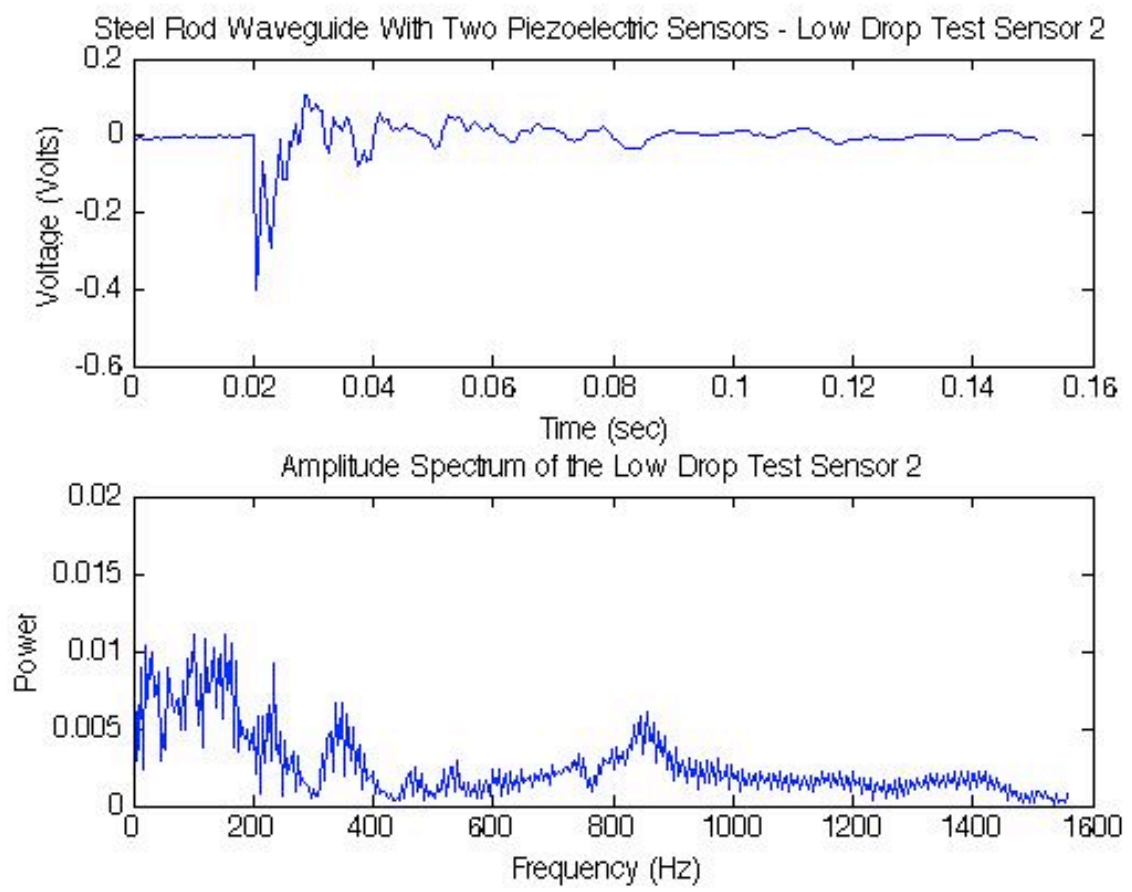

Figure 81: Results of the low drop test from piezoelectric sensor 2 

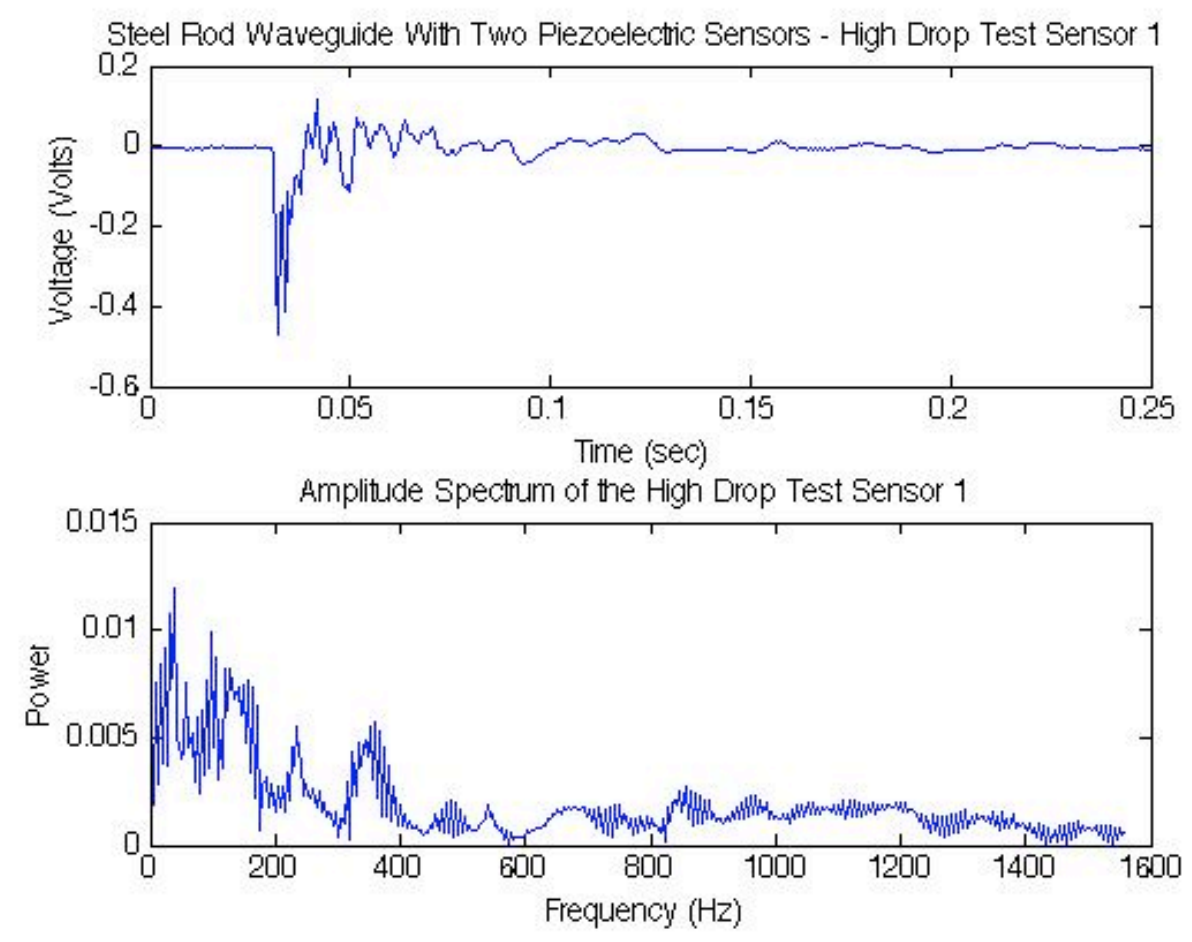

Figure 82: Results of the high drop test from piezoelectric sensor 1
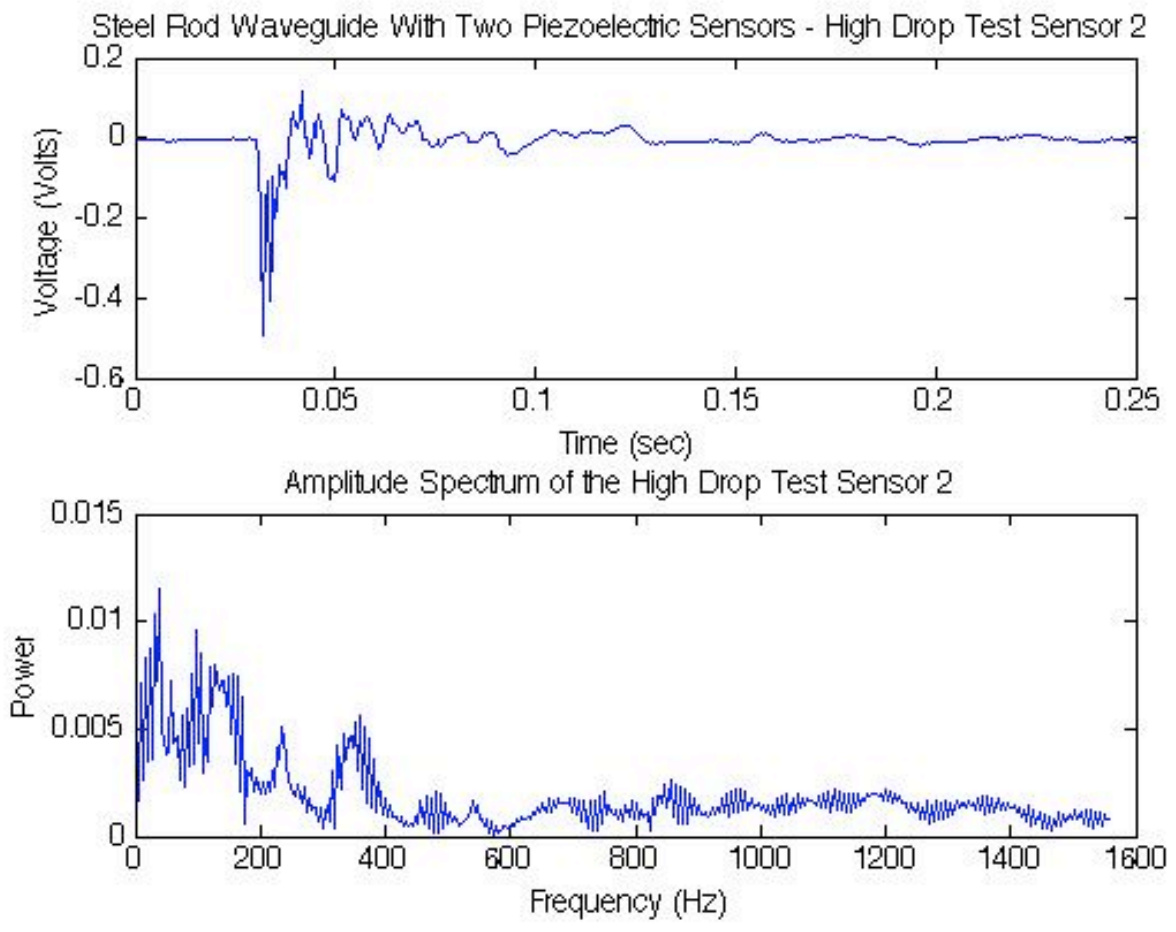

Figure 83: Results of the high drop test from piezoelectric sensor 2 


\subsubsection{Analysis}

Results appear in figures 81-84. Sensor one is triggered slightly before sensor two. The trigger time difference between sensor one and sensor two for the low drop test is approximately $2.5 \mu$ s. The trigger time difference between sensor one and sensor two for the high drop test is the same. The wave speeds of the two waves can be calculated with equation 17 from section 9.1.

The distance between the two sensors is approximately $3.96 \mathrm{~cm}$. The wave speed of the wave from the low drop test is calculated to be approximately $1585 \mathrm{~m} / \mathrm{s}$; the wave speed of the wave from the high drop test is equivalent. These results can be repeated with wave speed dependent upon amplitude.

\subsection{Comparison}

The sensors in the ball and steel rod waveguides are located in the same relative locations so the waveguide can be compared. The resulting peak voltages at sensor one for the low drop tests are nearly identical, but the resulting peak voltages at sensor two are different. The peak voltage from the steel ball high drop test at sensor two is $0.75 \mathrm{~V}$ while the peak voltage from the steel rod low drop test is only $0.5 \mathrm{~V}$. A comparison of the high drop tests reveals similar results. The power amplitude spectrum for the steel ball waveguide drop tests is more compact and of greater amplitude than that of the steel rod tests with a frequency peak at around $170 \mathrm{~Hz}$. With this being said it is also apparent from the results in 9.1.3 and 9.2.3 that the steel rod waveguide seems to transmit vibration at a high velocity than the steel ball waveguide by about a factor of ten. 


\section{CHPATER TEN: WAVEGUIDE ACTUATION}

\subsection{Steel Rod}

\subsubsection{Test Setup}

The steel rod fitted with two piezoelectric discs from 9.2 is secured in the same test-bed from figure 83. The sensors are actuated with the HP 33120A function generator from at $100 \mathrm{mV}$ with a $100 \mathrm{kHz}$ sine wave signal. The two signals excite the steel rod and $\mathrm{AE}$ data is collected at each end by two PAC sensors.

\subsubsection{Section Results}

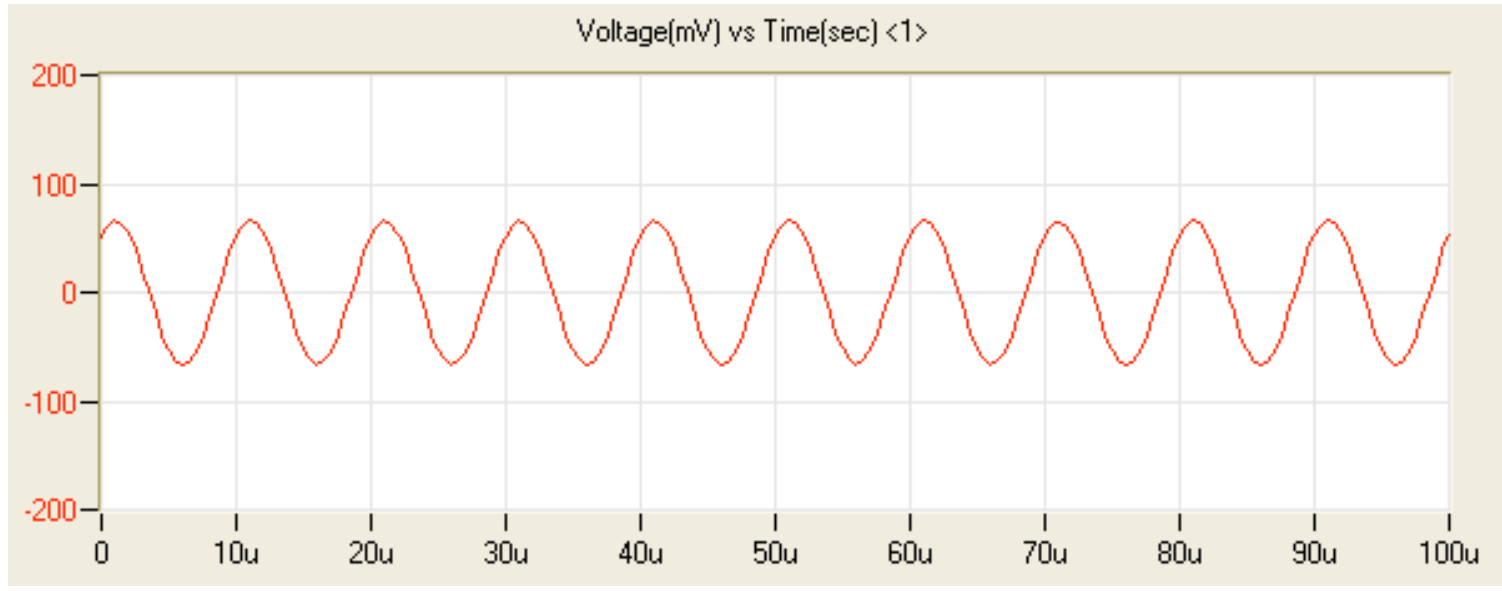

Figure 84: Resulting signal at PAC Sensor 1

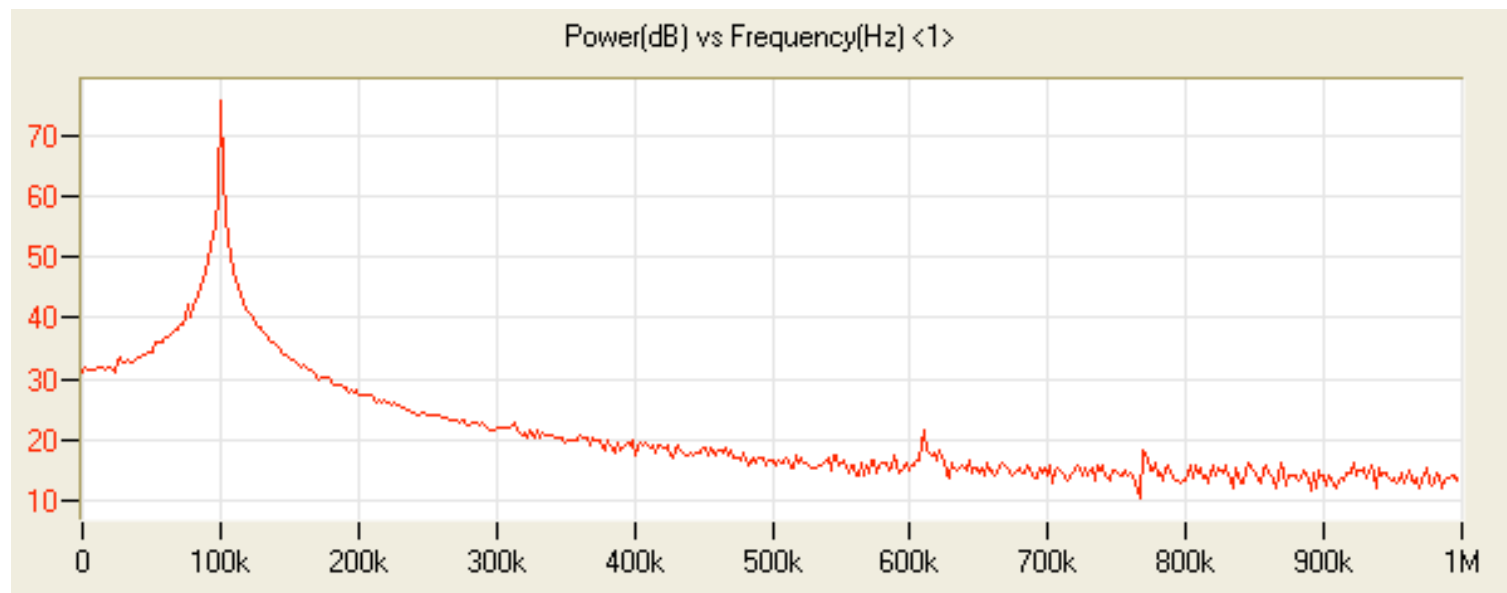

Figure 85: Resulting Fourier Transform of the signal in figure 84 


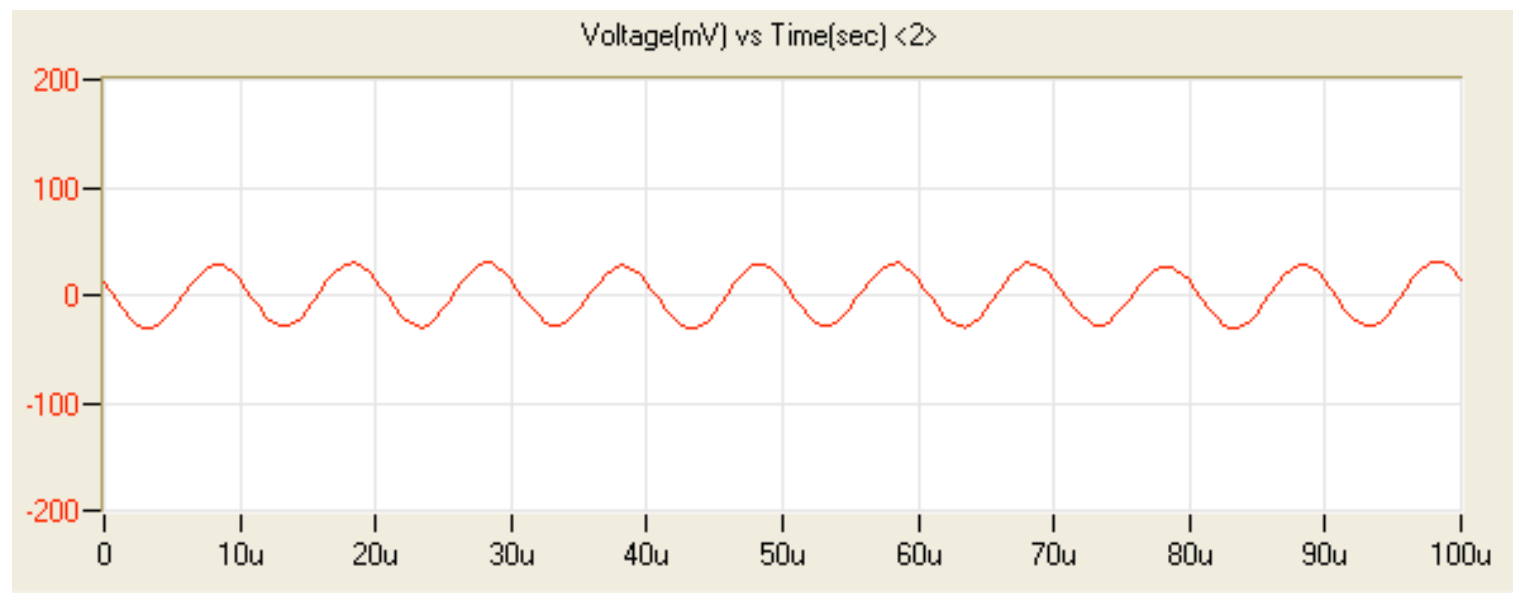

Figure 86: Resulting signal at PAC Sensor 2

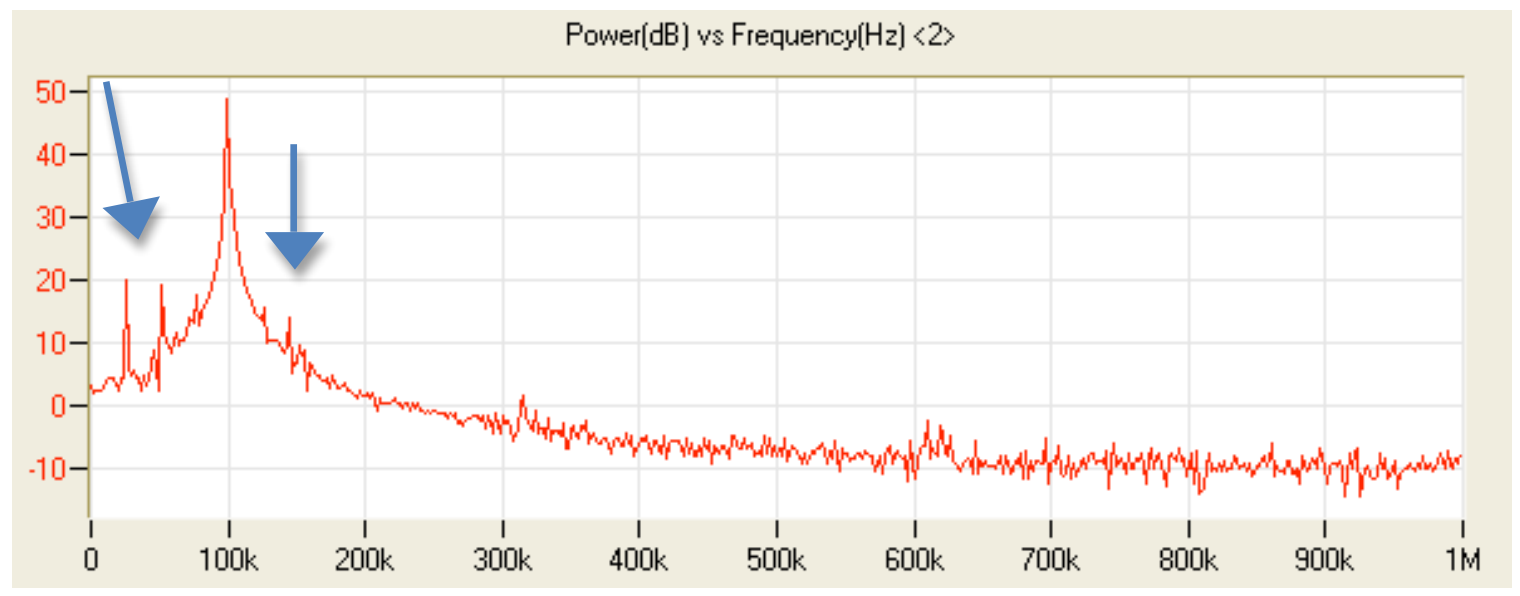

Figure 87: Resulting Fourier Transform of the signal in figure 86

Results appear in figures 84-87. The signal experienced by the first sensor is stronger than that experienced by the second presumably due to the testing rig damping the vibration experienced at the bottom end of the rod. Three peak frequencies occur around the $100 \mathrm{kHz}$ frequency peak highlighted with arrows in figure 87. 


\section{CHAPTER ELEVEN: CONCLUSION}

\subsection{Final Comments}

Steel ball nonlinear waveguide was fabricated for acoustic emissions testing. Steel balls were successfully modified with inline piezoelectric elements to sense elastodynamic waves. The result of pencil lead break tests reveal that the waveguide is sensitive to acoustic emission type waves. Small harmonic elastic waves do not propagate well through the waveguide, except at specific frequencies. A plasma lab AE acquisition could potentially exemplify the quality of these nonlinear waveguides.

The plasma torch trials, AE acquisition, and plasma composition classification can be considered a success; a MANOVA statistical analysis indicates a fair significance of the grouping [2]. The overall success of the classification points to the fact that we can someday classify AE data from different samples and from trials of differing plasma composition based on a database of past trials, immediately gaining statistical information of the trial with respect to a current database.

For future experimentation testing will commence for phenolic-impregnated carbon ablator (PICA) samples and silicon impregnated reusable ceramic ablator (SIRCA) samples with room for further sample experimentation [2]. Iterating through numerous different compositions of plasma and samples in sample plasma lab $\mathrm{AE}$ acquisition tests can help create an AE training data matrix for all samples. Current data can then be statistically compared to this matrix consisting of previous trials.

Future directions for research include using the nonlinear waveguide in high heat flux thermal protection system testing at UVM's Inductively Coupled Plasma Torch facility. 


\section{WORKS CITED}

[1] Graff, Karl F. Wave Motion In Elastic Solids. N.p.: Ohio State UP, 1975. Print.

[2] Hurley, D., Huston, D., \& Fletcher, Ds. Remote Monitoring of Harsh Environments using Waveguide Acoustic Emissions. Journal of Engineering Mechanics, Vol. 139, 3, 286-295. 2013.

[3] Hurley, David. Embedded and Remote Systems for Damage Detection Assessment, and Repair. Thesis. The University of Vermont, 2012. Print.

[4] Duda, Richard O., Peter E. Hart, and David G. Stork. Pattern Classification. New York: Wiley, 2001. Print.

[5] Porter, Mason A., Chiara Daraio, Ivan Szelengowicz, Eric B. Herbold, and P.g. Kevrekidis. "Highly Nonlinear Solitary Waves in Heterogeneous Periodic Particle Media." Physica D: Nonlinear Phenomena 238.6 (2009): 666-76. Print.

[6] Ni X, Rizzo P, Yang J, Katri D, \& Daraio C. Monitoring the hydration of cement using highly nonlinear solitary waves. NDT\&E Int (2012), http://dx.doi.org/10.1016/j.ndteint.2012.05.003

[7] Cambridge University Engineering Department. Materials Data Book. 2003 ed. http://www.mdp.eng.cam.ac.uk/web/library/enginfo/cueddatabooks/materials.pdf.

[8] Irvine, Tom. "DAMPING PROPERTIES OF MATERIALS Revision C." 2004. http://www.cs.wright.edu/ jslater/SDTCOutreachWebsite/damping\%20properties\%20of $\% 20$ materials.pdf.

[9] Herbold, E. B., J. Kim, V. F. Nesterenko, S. Y. Wang, and C. Daraio. "Pulse Propagation in a Linear and Nonlinear Diatomic Periodic Chain: Effects of Acoustic Frequency Band-gap." Acta Mech (2009): 85-103. Web. 30 Aug. 2013. 


\section{APPENDIX A: CALCULATIONS}

1. Accuracy of the measured center of the plate:

- $\sim / /$ - 1/16" (finest measurement seen by the human eye) in two directions

- Separated by an angle of $\sim 36^{\circ}+/-1^{\circ}$

○ Law of cosines: $c^{2}=a^{2}+b^{2}-2 a b \cos \theta$

○ Vertically:

- $+/-\sqrt{\left(\frac{1}{16}\right)^{2}+\left(\frac{1}{16}\right)^{2}-2\left(\frac{1}{16}\right)\left(\frac{1}{16}\right) \cos (37)}=+/-0.04 "$

○ Horizontally:

$$
\text { - }+/-\sqrt{\left(\frac{1}{16}\right)^{2}+\left(\frac{1}{16}\right)^{2}-2\left(\frac{1}{16}\right)\left(\frac{1}{16}\right) \cos (145)}=+/-0.12 ”
$$

- The actual center of the plate is within this interval surrounding the marked center with a $95 \%$ confidence 